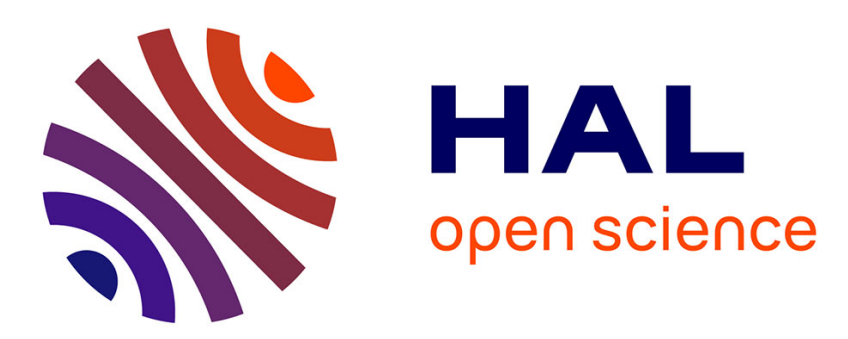

\title{
Le chien dans la religion et dans la vie quotidienne des communautés phéniciennes et puniques de la Méditerranée occidentale
}

\author{
Bruno D'andrea
}

\section{- To cite this version:}

Bruno D'andrea. Le chien dans la religion et dans la vie quotidienne des communautés phéniciennes et puniques de la Méditerranée occidentale. Mélanges de l'Ecole française de Rome - Antiquité, 2018, 130.1. hal-01908637

\section{HAL Id: hal-01908637 \\ https://hal.science/hal-01908637}

Submitted on 22 Jul 2020

HAL is a multi-disciplinary open access archive for the deposit and dissemination of scientific research documents, whether they are published or not. The documents may come from teaching and research institutions in France or abroad, or from public or private research centers.
L'archive ouverte pluridisciplinaire HAL, est destinée au dépôt et à la diffusion de documents scientifiques de niveau recherche, publiés ou non, émanant des établissements d'enseignement et de recherche français ou étrangers, des laboratoires publics ou privés. 


\title{
Le chien dans la religion et dans la vie quotidienne des communautés phéniciennes et puniques de la Méditerranée occidentale
}

\author{
Bruno D'ANDREA*
}

Br. D’Andrea, École française de Rome, bruno.dandrea@efrome.it

Le chien se caractérise par la pluralité de ses fonctions, laquelle engendre une diversité des valeurs symboliques qui lui sont associées et qui sont parfois contradictoires. La présente contribution vise à étudier les fonctions "concrètes" et symboliques du chien dans la religion phénicienne et punique à travers l'analyse des sources archéologiques, archéozoologiques, épigraphiques, iconographiques et littéraires. Pour atteindre cet objectif, il est nécessaire d'examiner plus généralement le rôle de cet animal dans la vie quotidienne, y compris l'épineuse question de la consommation de sa viande (cynophagie). La recherche sera focalisée sur les régions de la Méditerranée occidentale sujettes à l'expansion phénicienne pour la période comprise entre le $\mathrm{IX}^{\mathrm{e}}$ et le $\mathrm{II}^{\mathrm{e}}$ s. av. J.-C. Les données portant sur les Phéniciens "d'Orient" (Chypre et Levant), ainsi que celles des cultures méditerranéennes et proche-orientales avec lesquelles les Phéniciens ont interagi, seront également sollicitées dans une perspective comparatiste.

Animal studies, archéologie du rite, chien, cynophagie, Phéniciens/Puniques, Méditerranée occidentale, religion

A variety of functions has always characterized the dog, determining various symbolic meanings and contradictory conceptions. This paper aims at studying the religious meaning and use of dogs in the Phoenician and Punic religion, by considering the archeological, zooarchaeological, epigraphic, iconographic and textual evidence. In order to achieve this objective, the role of this animal in daily life and the thorny issue of dog meat consumption (cynophagia) are examined. This study focuses on the Phoenician/Punic regions in the Western Mediterranean during the $9^{\text {th }}-2^{\text {nd }}$ centuries BC. However, the "Phoenician" evidence from Cyprus and Levant, as well as the data from the other Mediterranean and Near Eastern cultures, will be also considered from a comparative perspective.

Animal studies, archaeology of ritual, cynophagia, dog, Phoenicians/Punics, West Mediterranean, religion

\section{INTRODUCTION}

La présente contribution, qui fait partie d'un projet de recherche en cours ${ }^{1}$, vise à étudier les fonctions "concrètes" et symboliques du chien dans la religion phénico-punique à travers l'analyse des sources archéologiques, archéozoologiques, épigraphiques, iconographiques et littéraires. Pour
* Ce travail a bénéficié du soutien du LabEx ARCHIMEDE (Montpellier) au titre du programme «Investissement d'Avenir" ANR-11-LABX-0032-01. Je remercie M. G. Amadasi Guzzo, N. Chiarenza et A. Nieto Espinet pour avoir accepté de relire mon texte, pour leurs conseils et remarques constructives. Je tiens à remercier tout particulièrement H. Normand, qui a assuré la relecture du texte français.

1. «L'animal dans la religion phénicienne de la Méditerranée occidentale et les effets de la conquête romaine" (projet en cours à l'École française de Rome sur lequel j'ai commencé à travailler au LabEx ARCHIMEDE en 2016-2017). 
Le chien dans la religion et dans la vie quotidienne des communautés phéniciennes et puniques

atteindre cet objectif, il est nécessaire d'examiner plus généralement le rôle de cet animal dans la vie quotidienne, y compris l'épineuse question de la cynophagie.

$\mathrm{Au}$ cours des dernières années, plusieurs études se sont concentrées sur le chien, essentiellement dans le monde classique de la Méditerranée occidentale et au Proche-Orient ancien, traitant de questions particulières ou proposant une approche plus globale du rôle de l'animal ${ }^{2}$. Ces travaux ont été consultés lors de la préparation de cette contribution de manière à adopter un cadre théorique et méthodologique approprié et à favoriser une approche comparative. Quant aux études sur la culture phénico-punique, A.M. Niveau de Villedary s'est occupée du chien dans deux articles, dont l'un a été publié en collaboration avec E. Ferrer Albelda ${ }^{3}$ : des trouvailles de la nécropole punique de Cadix ont fourni l'occasion d'examiner la présence et le rôle de cet animal dans les contextes rituels. D'autres travaux ont traité de thèmes spécifiques: la cynophagie pratiquée par les Phéniciens et les Carthaginois"; les "sépultures/nécropoles" de chiens dans la région levantine à l'âge du Fer et à l'époque perse ${ }^{5}$; les représentations iconographiques des animaux à Carthage ${ }^{6}$. Ces travaux sont des références primordiales pour cette étude.

Le chien se caractérise par la pluralité de ses fonctions, laquelle engendre une diversité des valeurs symboliques qui lui sont associées et qui sont parfois contradictoires. Il peut être consommé par l'homme mais il est surtout utilisé pour la chasse, la garde des troupeaux, la protection des personnes, des biens et des espaces, la guerre, le transport de charges, le nettoyage (par la consommation des déchets alimentaires), ou encore pour servir d'animal de compagnie ( "pet»). Il vit dans les mêmes espaces que l'homme, dont il est le

2. Voir la note 8. Parmi ces travaux, il y a aussi plusieurs monographies et ouvrages collectifs: Brewer - Clark Philips 2001 ; Moore - Snyder 2006.

3. Ferrer Albelda - Niveau de Villedary 2004; Niveau de Villedary 2008.

4. Cardoso-Gomes 1997.

5. Voir les notes 49-51.

6. Yazidi 2009. commensal et un concurrent potentiel, à l'état domestique ou semi-sauvage ( paria»). L'homme apprécie certaines de ses caractéristiques comportementales (fidélité, capacité de reconnaître son maître, etc.), mais d'autres caractéristiques (copulation en public, nécrophagie/anthropophagie, coprophagie, etc.) concourent à l'image d'un animal «senza ritegno", comme le dit C. Franco dans son excellent travail sur l'image du chien et de la femme dans la Grèce ancienne ${ }^{7}$. Cet animal se prête donc à des conceptions binaires, souvent ambivalentes (agressivité/mansuétude, chtonien/ céleste, domestique/sauvage, monde des vivants/ monde des morts, pur/impur) et ambiguës comme le sont les divinités auxquelles il est associé ou sacrifié (généralement, il s'agit de divinités liées aux limites et aux moments de passage comme $l^{\prime}$ accouchement, la maladie et la mort) ${ }^{8}$.

Cependant, le mot chien peut indiquer plusieurs types de chiens, qui diffèrent selon leur statut (domestique/paria/sauvage, pet/garde/ «competitor», etc.), leur race, leur sexe, leur taille, leur âge et aussi, dans certaines pratiques rituelles, leur couleur'. Dans les travaux sur les religions de la Méditerranée et du Proche-Orient, les chiens sont associés de façon récurrente aux rites de purification, d'expiation et de passage, en raison de leur ambivalence et de leur prétendue impureté. Mais ces associations sont souvent le résultat de généralisations développées à partir de cas spécifiques concernant un type de chien; pire encore, elles découlent parfois de raisonnements circulaires fondés sur des a priori, comme l'impureté constitutive et la connotation négative du chien, qui sont loin d'être prouvés ${ }^{10}$.

Les travaux de C. Franco sont très intéressants à ce $\operatorname{propos}^{11}$ : en analysant les nombreuses insultes associées au chien dans la Grèce ancienne, la chercheuse estime que celles-ci n'indiquent

7. Franco 2003.

8. Collins 1992 (monde hittite); Lacam 2008; Luce 2008; M. Mackinnon dans Campbell 2014, p. 269-281; Luce 2015 (monde gréco-romain); Horard-Herbin 2014 (monde celtique-gaulois); Raptou 2009 (Chypre); Ornan 2004; Miller 2008; Meir 2013 (Levant et Proche-Orient); Colin - Adam - Pranjic 2014 (Égypte).

9. Des chiens roux étaient sacrifiés à Rome lors de l'Augurium canarium: Lacam 2008, p. 43. Dans le monde grec, de jeunes chiens étaient généralement utilisés dans les rites apotropaïques: Georgoudi 2015.

10. Pour une approche critique cf. Miller 2008 (Levant); Georgoudi 2015 (monde grec).

11. Franco 2003. 
pas nécessairement du mépris envers cet animal, mais qu'elles sont plutôt liées à la contiguïté entre l'homme et le chien (qui serait un animal métonymique au sens lévi-straussien du terme). Au-dessous de l'homme mais au-dessus des autres animaux, le chien aurait occupé une position ambiguë et marginale, semblable à celle des prostitués, des esclaves et des cochons, auxquels il est souvent associé. Ces individus se prêteraient aux insultes, non seulement par leurs caractéristiques, mais aussi - et surtout - par leur "position sociale». Ainsi, les expressions dans lesquelles le chien est impliqué peuvent se révéler trompeuses pour qui cherche à reconstituer son image.

\section{CADRE GÉOGRAPHIQUE ET CHRONOLOGIQUE}

Comme le rappellent des travaux récents ${ }^{12}$, la définition d'un "monde" phénicien et punique n'est ni évidente ni aisée. En effet, les mots Phénicie et Phéniciens n'indiquent pas une nation ou un ethnos mais désignent une réalité culturelle et géographique mouvante, caractérisée par certains éléments culturels et matériels qui lui sont propres $^{13}$. Ces traits distinctifs justifient, à mon sens, l'utilisation du terme phénicien/punique, mais il faut éviter une conception monolithique de cette "civilisation". L'application d'étiquettes ethno-culturelles aux sites et aux contextes archéologiques s'avère souvent problématique, surtout dans des contextes particulièrement ouverts et dynamiques comme le sont les communautés «coloniales» phéniciennes de la Méditerranée occidentale. À ce propos, l'étude du rôle et de l'image des animaux, surtout dans la sphère reli-

12. Crawley Quinn - Vella 2014; Xella 2014; Garbati - Pedrazzi 2015 et 2016; Quinn 2017.

13. Le même constat est valable pour la plupart des «civilisations " anciennes, par ex. pour les Étrusques. Dès lors, c'est l'appartenance culturelle qui est centrale: les Phéniciens ne se nomment jamais comme tels, mais par leur appartenance à leur cité (comme c'est le cas, d'ailleurs, chez les Grecs...) ; en revanche, rien ne prouve qu'ils ne se sentaient pas Phéniciens car partageant des éléments communs (culture matérielle, langue, religion, etc.) et se différenciant des autres civilisations (qui, comme les Grecs et les Romains, les identifiaient en tant que Phéniciens/Puniques). Il est très probable qu'une construction identitaire phénicienne ait été développée (réinventée) à nouveau dans les "colonies » de la Méditerranée occidentale comme un moyen de différenciation entre «nous» et les «autres». gieuse, nous offre des éléments de réflexion sur les questions de l' «identité», du mélange ethnique et culturel et de l'hétérogénéité entre les différentes communautés phénico-puniques.

Cette contribution se focalise sur les régions de la Méditerranée occidentale sujettes à l'expansion phénicienne pour la période comprise entre le IX $\mathrm{s}$. lorsque les premiers établissements phéniciens sont fondés, et le II ${ }^{\mathrm{e}} \mathrm{s}{ }^{14}$. Les données portant sur les Phéniciens "d'Orient" (Chypre et Levant) seront également sollicitées dans une perspective comparatiste. Le terme Punique a tendance à remplacer celui de Phénicien pour désigner les Phéniciens de la Méditerranée occidentale à partir du $\mathrm{VI}^{\mathrm{e}}-\mathrm{V}^{\mathrm{e}} \mathrm{s}$, époque où Carthage commence à s'imposer aux autres colonies phéniciennes d'Occident ${ }^{15}$.

\section{LES SOURCES ÉPIGRAPHIQUES ET LITTÉRAIRES}

Les sources épigraphiques et littéraires donnant des informations sur le chien dans le monde phénico-punique sont peu nombreuses et parfois indirectes ou extérieures. Le plus ancien texte en langue phénicienne dans lequel on trouve le terme KLB (chien) est l'inscription monumentale du roi Kulamuwa de Sam'al (Zinçirli) datée de la fin du $\mathrm{IX}^{\mathrm{e}} \mathrm{s}^{16}$. Kulamuwa dit que, sous les rois précédents, un groupe de citoyens avaient été traités/soumis "comme des chiens"; le zoonyme semble utilisé ici de façon péjorative.

Des chiens (KLBM) sont mentionnés dans une inscription phénicienne peinte sur une plaque de pierre retrouvée à Kition (Larnaca) et datée de la moitié du Ve s. environ ${ }^{17}$. L'inscription établit les rémunérations du personnel du temple d'Astarté et les KLBM font partie du personnel. Selon la plupart des chercheurs, il s'agirait d'hommes voués au service de la déesse en tant que prostitués ${ }^{18}$.

14. Dans la suite du texte et sauf mention expresse du contraire, toutes les dates s'entendent avant J.-C.

15. Melliti 2016, p. 65-92. La thèse d'une politique impérialiste de Carthage déjà pendant le $\mathrm{VI}^{\mathrm{e}} \mathrm{s}$. reste ouverte et débattue.

16. KAI 24

17. $K A I 37=$ CIS 86. Cf. Peckham 1968.

18. Hoftijzer - Jongeling 1995, p. 509. KLBM est associé à GRM, qui vraisemblablement indique lui aussi une catégorie de personnel du temple; toutefois, son interprétation reste douteuse ("jeunes prostitués», "clients», "lions»). Sur ces thèses et sur la prostitution sacrée: Lipiński 1995, p. 486-489; Ribichini 2004a. 
Le chien dans la religion et dans la vie quotidienne des communautés phéniciennes et puniques

L'hypothèse se fonde sur trois considérations.

- Dans la même inscription sont citées des 'LMT, généralement interprétées comme des prostituées sacrées.

- Si les KLBM étaient effectivement des chiens, pourquoi auraient-ils reçu un salaire?

- Une association entre des chiens, la prostitution et des prostitués se retrouve dans un passage du Deutéronome qui, juste après avoir mentionné des femmes et des hommes prostitués, met en parallèle le salaire d'une prostituée et le prix d'un chien comme des symboles d'abomination ${ }^{19}$.

D'autres chercheurs ont proposé des interprétations différentes: pour certains, il s'agissait d'hommes qui participaient aux cérémonies en portant des masques de chiens ${ }^{20}$; pour d'autres, d'animaux consacrés à la divinité et qui vivaient dans le sanctuaire ${ }^{21}$. Des exemples de chiens demeurant dans des sanctuaires sont effectivement connus dans le monde gréco-romain et au Proche-Orient ${ }^{22}$. Néanmoins, dans l'inscription de Kition, l'existence d'un salaire reste difficile à expliquer. La thèse des prostitués paraît la plus vraisemblable, mais elle s'appuie sur un raisonnement circulaire en considérant que l'interprétation des 'LMT comme des prostituées sacrées et des KLBM du Deutéronome comme des pédérastes demeurent incertaines. De toute façon, les KLBM de Kition seraient des individus au service d'Astarté et ce terme n'aurait pas nécessairement de connotation péjorative mais pourrait, bien au contraire, exprimer l'attachement et la fidélité à la divinité.

Une image positive de dévouement aux dieux semble être présente dans le théophore KLB'LM et dans les hypocoristiques KLB', KLBL' et KLBY (dans un cas on trouve le féminin KLBT) qui sont

19. Dt. 23, 18-19. Toutefois, Flavius Josèphe interprète littéralement le terme chien (Antiquités juives, 8, 9).

20. Peckham 1968, p. 317.

21. L.E. Stager dans Stager 2008, p. 567. Cf., à ce propos, l'interprétation proposée pour les chiens découverts à Paphos (Chypre) en association avec des sépultures humaines d'époque hellénistique et romaine: Raptou 2009.

22. On peut citer les chiens sacrés égyptiens (Hdt., 6, 66-67; Diodore, Bibl., 1, 83-84; cf. Colin - Adam - Pranjic 2014), les chiens du sanctuaire grec d'Adranos en Sicile (Élien, $N A, 11,20)$, les chiens utilisés dans les rites de guérison des Asclépiéia (K. Trantalidou dans Moore - Snyder 2006, p. 114; Luce 2008, p. 283-285; Raptou 2009, p. 105-111) et les chiens du temple mésopotamien de Gula à Isin (Ornan 2004). attestés, quoique rarement, dans l'onomastique phénico-punique ${ }^{23}$.

C'est un chien qui est le protagoniste du mythe relatif à la découverte de la pourpre - un mythe dont il existe plusieurs récits d'époque tardive (II ${ }^{\text {- }}$ $\mathrm{VI}^{\mathrm{e}}$ s. apr. J.-C.), parfois discordants: la scène se déroule à Tyr et le chien accompagne un berger (selon Julius Pollux il s'agit du « chien d'Héraclès"; ce dernier est notoirement identifié avec Melqart, le dieu éponyme de la ville) ${ }^{24}$. L'association, sur certaines monnaies de Tyr d'époque impériale, d'un chien et d'un murex paraît confirmer l'origine locale et phénicienne de ce mythe ${ }^{25}$.

Justin narre qu'à la vielle de la première guerre médique, vers 492, le roi Darius avait envoyé une ambassade aux Carthaginois pour leur demander leur soutien contre la Grèce et, en même temps, pour leur interdire de se nourrir de chiens, de faire des sacrifices humains et d'inhumer les défunts ${ }^{26}$. Les Carthaginois auraient refusé de participer à la guerre mais auraient accepté les autres prescriptions. Ce récit est constamment évoqué par ceux qui ont abordé la question de la cynophagie phénico-punique ${ }^{27}$. Toutefois, comme le dit s. Ribichini, il faut considérer qu'il pose différents problèmes « tali da suggerire che si tratti di un passo apocrifo $"^{28}$. En tout cas, Justin fait référence uniquement à Carthage (qui, à cette période, contrôlait la plupart des établissements phéniciens de Sardaigne et de Sicile), sans mentionner les Phéniciens d'Orient alors qu'à cette époque, ceux-ci étaient soumis aux Perses. La cynophagie, associée aux sacrifices humains, semble avoir ici une connotation péjorative: c'est peut-être la raison pour laquelle elle est imputée aux Carthaginois.

La rareté des mentions de cet animal dans la documentation épigraphique et littéraire est inté-

23. CIS 49 et 52 (Kition); 702, 4657, 4711, 5040, 5772 (Carthage); Amadasi Guzzo 1986, p. 35-36, nº 30 (Motyé); Sader 2005, p. 63, no 41 (Tyr); Jongeling 2008, p. 215 (Constantine).

24. Cf. Achille Tatius, Leucippé et Clitophon, 1, 11; Grégoire de Nazianze, Orationes, 4, 108; Julius Pollux, Onomasticon, 1, 45-46.

25. Naster 1985.

26. Justin, 19, 1.

27. Cardoso - Gomes 1997, p. 89; Niveau de Villedary 2008, p. 115 ; Campanella 2008, p. 70 ; Yazidi 2009, p. 303.

28. Ribichini 1998, p. 656-658. 
ressante en soi, mais il faut dire que ces sources ne donnent pas non plus assez d'informations à propos d'autres animaux. Le chien n'est pas mentionné dans les "tarifs» carthaginois, lesquels précisent quelques espèces animales sacrifiées ${ }^{29}$. $\mathrm{Au}$ contraire, les sources littéraires gréco-latines donnent plusieurs renseignements sur le rôle religieux du chien dans leur propre culture ${ }^{30}$ et dans des cultures environnantes comme celle de l'Égypte $^{31}$. Plusieurs textes grecs, latins et hiéroglyphiques mentionnent des chiens et certains d'entre eux indiquent que l'animal avait fait l'objet d'une sépulture ou avait été enterré avec son maître ${ }^{32}$.

La documentation ougaritique et hébraïque/ vétérotestamentaire peut donner des renseignements intéressants, si l'on considère sa proximité géographique et culturelle avec les Phéniciens d'Orient. Elle doit cependant être utilisée seulement à titre de comparaison. Le mot chien (en ougaritique klb, "chien», inr, probablement "chien bâtard", et irn, "chiot») est attesté dans plusieurs textes ougaritiques, parmi lesquels figurent des textes à la fois mythologiques et religieux $^{33}$. D'une part, cet animal y est associé à des personnages divins, héroïques et humains en tant que chien de chasse ou comme "commensal» durant les banquets; d'autre part, il est utilisé dans quelques expressions au sens figuré, généralement en tant que symbole d'asservissement (avec une connotation soit péjorative soit positive). Le chien n'est jamais cité dans des scènes de rites sacrificiels, alors que son utilisation est bien attestée dans les textes de magie et de pharmacopée ${ }^{34}$. Dans des lettres du XIII ${ }^{\mathrm{e}}$ s. envoyées par le roi de Sidon au roi d'Ougarit, le premier qualifie de chiens (avec un sens évidemment dépréciatif) des citoyens ougaritiques qui avaient profané un temple de la ville de Sidon ${ }^{35}$.

Dans quelques inscriptions hébraïques ${ }^{36}$ et dans plusieurs textes bibliques, le zoonyme est employé comme symbole d'asservissement. Dans l'Ancien et le Nouveau Testaments, l'appellation chien (quelquefois "chien mort" ou "tête de chien») a souvent une connotation péjorative et insultante $^{37}$. À plusieurs reprises, l'animal est vu comme un objet de répulsion, certaines de ses particularités comportementales étant considérées comme des "abominations pour le Seigneur»: la nécrophagie, l'agressivité, le fait de retourner à ce qu'il a vomi, de lécher le sang, etc. Dans un passage du livre de Job, le chien est associé au gardiennage des troupeaux ${ }^{38}$. Il est intéressant de noter que cet animal ne fait partie ni des animaux qu'on peut sacrifier au "Seigneur" ni des animaux impurs mentionnés dans le Lévitique. En revanche, dans un passage du livre d'Isaïe (66 3), le meurtre d'un chien est considéré une "abomination".

Les textes ougaritiques et hébraïco-bibliques révèlent sans doute une familiarité avec le chien: l'utilisation du zoonyme dans des insultes ou comme symbole de certains comportements le démontrent. Cela ne signifie pas nécessairement qu'il y avait une attitude négative à l'égard de cet animal ni que celui-ci était perçu comme impur, même si l'idée d'une connotation négative du chien dans le "monde sémitique " est répandue.
29. CIS 165, $167=$ KAI 69, 74; CIS 3915-3917. Cf. Lipiński 1995, p. 466-474; O. Borowski dans Collins 2002, p. 415; D'Andrea (à paraître).

30. Le chien est sacrifié surtout dans des rites apotropaïques associés à des divinités liminaires. Dans certains cas, il est utilisé dans des rites dichotomiques ou divinatoires, parfois aussi dans des sacrifices caractérisés par le partage de la viande entre $1(\mathrm{es}) \mathrm{homme}(\mathrm{s})$ et le(s) dieu(x): Pline, $N H$, 29, 14; Plutarque, QR, 52, 68 et 111 ; Rom., 21, 10; Moralia, 280C, 290D. Cf. Franco 2003; Lacam 2008; Luce 2008; Raptou 2009, p. 104.

31. Voir la note 22.

32. Bouvier-Closse 2001; J. Brewer dans Brewer - Clark Philips 2001, p. 43-46; Luce 2008; 2015.

33. B.R. Foster dans Collins 2002, p. 301-303; Sumaka'i Fink 2003. Cf. Del Olmo Lete - Sanmartín 2003, p. 83, 104, 438-439.

34. Sumaka'i Fink 2003, p. 38-39 et 49-51. L'utilisation - et parfois la consommation - du chien à des fins médicales et thérapeutiques sont attestées dans les mondes égyptien (J. Brewer dans Brewer - Clark - Philips 2001, p. 44), gréco-romain (Pline, NH, 29-30; cf. V. Goebel et J. Peters dans Campbell 2014, p. 601), hittite (Collins 1992) et proche-oriental (Ornan 2004; Schattner-Rieser 2009, p. 303), ainsi qu'au Maghreb à l'époque moderne et contemporaine (Prevost 2006).

35. Sumaka'i Fink 2003, p. 50 (RS 86.2221, 18.054).

36. KAI 192, 195, 196: Hoftijzer - Jongieling 1995, p. 509.

37. IR $1411,164,2119,2123-24,2228 ; 2 \mathrm{R} 813,910,9$ 36 ; Jr 153 ; $1 \mathrm{~S} 1743,2415 ; 2 \mathrm{~S} 38,98,169$; Is $5610-11$; Pr 2611 ; Ps 68 24; 2Pi 22 ; Apoc 22 15; Phil 32 ; Matt 7 6. Cf. Miller 2008; Schattner-Rieser 2009.

38. Job 301. 
Le chien dans la religion et dans la vie quotidienne des communautés phéniciennes et puniques

\section{LES SOURCES ARCHÉOZOOLOGIQUES ET ARCHÉOLOGIQUES}

Les données du monde phénicien d'Orient et le phénomène des «sépultures/ nécropoles» de chiens

Les études archéozoologiques relatives aux établissements phéniciens du Levant et de Chypre au Bronze récent et à l'âge du Fer sont peu nombreuses: le chien est représenté de façon faible et il est quelquefois totalement absent ${ }^{39}$. Le même constat est valable pour toute la région levantine et, plus généralement, pour le Proche-Orient à partir du Néolithique jusqu'à l'époque romaine. La consommation du chien est attestée de façon très sporadique $^{40}$, et jamais dans des établissements phéniciens. Quelques restes de Canis familiaris ont été retrouvés dans les aires sacrées de Tell Kazel et de Sidon (Bronze récent) ${ }^{41}$, au Levant, et dans les sanctuaires de Kition Bamboula $\left(\mathrm{X}^{\mathrm{e}}-\mathrm{IV}^{\mathrm{e}} \mathrm{s} \text {. }\right)^{42}$ et Kathari $\left(\mathrm{IX}^{\mathrm{e}}-\mathrm{IV}^{\mathrm{e}} \mathrm{s} .\right)^{43}$, à Chypre, mais il n'y a pas de données plus précises sur leurs contextes de provenance.

Les nécropoles fournissent quelques renseignements intéressants: vers la fin du XIX ${ }^{\mathrm{e}} \mathrm{s}$, dans la nécropole royale de Sidon, sept crânes de chiens ( "lévriers") ont été découverts à côté du défunt dans un sarcophage placé dans une chambre funéraire du $\mathrm{IV}^{\mathrm{e}} \mathrm{s}{ }^{44}$ : il s'agissait du célèbre sarcophage "des pleureuses», qui est traditionnellement attribué au roi Straton $\mathrm{I}^{\text {er45 }}$. Voulant se détacher de l'empire perse, ce roi avait participé à la révolte des satrapes; après l'échec de l'entreprise, hésitant à se suicider, il avait été tué par sa femme ${ }^{46}$. Le décor du sarcophage est caractérisé par des scènes de chasse

39. Beyrouth (XVIII e s. av. - II e s. apr. J.-C.; pas des restes de Canis sur 188 restes déterminés, RD, en considérant seulement les mammifères: Rakham 1996), Dor (XII ${ }^{e}-\mathrm{VIII}^{\mathrm{e}}$ s., $0,2 \%$ sur 1878 RD: Raban-Gerstel et al. 2008), Kamid el-Loz (XV'-XI ${ }^{e}$ s., 2,8 \% sur 12000 RD environ: Bökönyi 1990), Tell el-Burak (XV'-XII ${ }^{\text {e }}$ s., 1,5\% sur $524 \mathrm{RD}$ ) et Ougarit (XIV ${ }^{e}-X I^{e}$ S., $0,2 \%$ sur 3662 RD: Vila 2008). Pour les données des sites libanais à l'âge du Bronze: Chahoud Vila 2011

40. Par ex. Wilkens 2000, p. 44 (Tell Afis).

41. $0,3 \%$ sur 1668 RD à Tell Kazel, 0,4 \% sur 448 RD à Sidon: Chahoud 2015.

42. 11 restes sur 184 RD: Gardeisen - Garcia Petit - Piquès 2015.

43. Un reste sur 1328 RD : Nobis 2000.

44. Hamdi Bey - Reinach 1892, p. 23-30.

45. Hamdi Bey - Reinach 1892, p. 238-271.

46. Diodore, Bibl., 15, 20; Jérôme, Contre Jovinien, 1, 45. dans lesquelles des lévriers sont impliqués, mais il est impossible de fixer un lien entre ces représentations et les crânes déposés dans la tombe. Ces crânes pourraient faire référence au statut royal du défunt (les chiens seraient les gardiens de la sépulture et/ou accompagneraient le défunt) ou aux évènements qui ont causé sa mort (les chiens auraient été utilisés dans des rites d'expiation-purification liés à sa "mort atypique»).

En 1963, au cours des fouilles de la nécropole de Khaldé $\left(\mathrm{X}^{\mathrm{e}}-\mathrm{VII}{ }^{\mathrm{e}} \mathrm{s}\right.$.), R. Saidah a découvert huit chiens enterrés à proximité des sépultures (surtout des inhumations) ${ }^{47}$. L'archéologue propose une interprétation rituelle de la présence de ces animaux en considérant leur "inhumation soigneuse", mais il ne donne pas de renseignements spécifiques sur le contexte. Le fémur d'un chien de plus de deux ans avec des traces de désarticulation a été retrouvé dans la nécropole de Tyr Al-Bass (des crémations secondaires datées de la fin du IX à la fin du VII ${ }^{\mathrm{e}}$ s.), fouillée au cours de cette dernière décennie par une équipe catalane dirigée par M.E. Aubet ${ }^{48}$. Ce fémur ne paraît pas associé à une sépulture spécifique.

Au cours du $\mathrm{I}^{\mathrm{er}}$ millénaire et surtout à l'époque perse, des sépultures/nécropoles de chiens ont été fouillées dans plusieurs établissements du Levant ${ }^{49}$, y compris dans quelques sites phéniciens (Beyrouth $^{50}$ et Tell el-Burak ${ }^{51}$; on peut ajouter le cas susmentionné de Khaldé, où toutefois les animaux sont associés à des sépultures humaines). Ces chiens avaient été déposés intentionnellement et avec soin (généralement dans des fosses) et, sauf dans de rares cas $^{52}$, leurs restes n'avaient pas de trace de consommation ni d'abattage. À Ashkelon,

47. Saidah 1967, p. 166.

48. M. Montero dans Aubet - Núñez-Trellisó 2014, p. 482-483.

49. P. Wapnish et B. Hesse dans Stager 2008, p. 559-560; Meir 2013; Çakirlar et al. 2013; Horwitz 2015. Cf. les sépultures de chiens découvertes à Paphos: voir la note 21.

50. P. Jablonka dans Finkbeiner et al. 1997, p. 130-132. Il s'agit de sept chiens enterrés dans des fosses peu profondes placées à l'intérieur du rempart; dans trois cas, des tessons de céramique avaient été déposés sur les squelettes.

51. Çakirlar et al. 2013. Deux chiens avaient été déposés dans une unité domestique, dans un contexte apparemment lié à sa destruction et au nivèlement des ruines; le premier avait six mois environ et se trouvait au-dessous d'un vase fragmentaire; le deuxième avait deux ans environ et son squelette n'était pas entier, probablement à cause de travaux d'excavations récents.

52. Horwitz 2015, p. 147 (traces de découpe, désarticulation et brûlure). 
600-700 chiens avaient été inhumés dans le même espace au $\mathrm{V}^{\mathrm{e}}-\mathrm{IV}^{\mathrm{e}} \mathrm{s}$. sur une période de quelques années $^{53}$ : leurs profils d'âge démontrent qu'il ne s'agissait pas d'un événement catastrophique ni soudain et que ces animaux n'avaient pas été sélectionnés en fonction de leur âge ou de leur sexe.

L'interprétation de ces inhumations reste débattue. Selon certains, les chiens avaient été sacrifiés avec des méthodes qui ne laissaient pas de traces sur les ossements (comme l'asphyxie) ${ }^{54}$; selon d'autres, ils étaient morts naturellement ${ }^{55}$. Pour ce qui concerne l'origine du phénomène, on a proposé, entre autres, les Phéniciens, les Perses ou une provenance égéo-anatolienne ${ }^{56}$. En réalité, ce dossier comprend des contextes et des dépôts différents et hétérogènes. Le dépôt d'un certain nombre de squelettes canins entiers et en connexion anatomique sur une période de quelques années (comme à Ashkelon) est intrinsèquement différent du dépôt unitaire et contemporain d'un ou plusieurs chiens (comme à Tell el-Burak), surtout quand ils sont retrouvés dans des structures ou contextes particuliers (des remparts, comme à Beyrouth; des nécropoles, comme à Khaldé; etc.). En outre, dans quelques cas, les chiens sont représentés seulement par des portions de squelette et/ ou ils sont associés à d'autres animaux.

En revanche, la "nécropole" d'Ashkelon reste un cas très intéressant, quoique difficile à expliquer. S'appuyant essentiellement sur l'inscription de Kition dont il a déjà été question, L.E. Stager considère qu'il s'agit de chiens consacrés à la divinité qui avaient vécu dans un sanctuaire (non localisé) placé à proximité, en tant que chiens consacrés à un dieu guérisseur identifié à Reshef-Mukol (le dieu MKL est cité dans l'inscription de Kition) ${ }^{57}$.
Cette thèse repose non sur des données concrètes mais plutôt sur des interprétations discutables et sur un raisonnement circulaire, même si la présence et l'influence phéniciennes à Ashkelon au cours de l'époque perse sont bien évidentes. En tout cas, dans le monde phénicien il n’y a pas de données qui témoigneraient d'un statut sacré du chien; en outre, les chiens d'Ashkelon étaient pour la plupart des chiots (62\%) et, au cours de leur vie, ils n'avait pas reçu de soins particuliers comme on s'y attendrait pour des animaux sacrés ${ }^{58}$. Tout en restant la plus vraisemblable, l'interprétation rituelle des inhumations canines d'Ashkelon ne peut pas être assurée, si l'on considère l'absence apparente d'une sélection des animaux, ainsi que l'absence de trace liée à leur abattage rituel et d'éléments indiquant qu'un statut particulier leur était attribué59.

Les assemblages fauniques des établissements phénico-puniques de la Méditerranée occidentale et la question de la cynophagie

Dans la fig. I figurent les restes de Canis familiaris des établissements phénico-puniques de la Méditerranée occidentale documentés par un minimum de 300 restes déterminés (NRD). Pour certains établissements, comme Alcáçoba de Santarem, Castro Marim, Huelva, Lisbonne et Nuraghe Sirai, l'attribution d'une étiquette ethno-culturelle phénicienne/punique (plutôt qu'indigène) reste problématique. D'une manière générale, les pourcentages de chiens sont toujours très bas, environ $1 \%$ en moyenne, conformément aux pourcentages qu'on retrouve dans la plupart
53. P. Wapnish et B. Hesse dans Stager 2008, p. 541-564.

54. Meir 2008, p. 269-270; Horwitz 2015, p. 148.

55. P. Wapnish et B. Hesse dans Stager 2008, p. 547-548 et 563-564. Les profils d'âge de chiens d'Ashkelon sont semblables aux profils des chiens morts naturellement; sur les ossements, il n'y avait pas de traces liées à une mort violente.

56. L.E. Stager dans Stager 2008, p. 565-568; Meir 2008; 2013; Horwitz 2015.

57. L.E. Stager dans Stager 2008, p. 565-568. L'archéologue propose une comparaison avec les sépultures des chiens du temple de Gula (Mésopotamie) à Isin de la fin du $\mathrm{XI}^{\mathrm{e}} \mathrm{s}$. (note 22). Il s'agit d'une comparaison difficile à accepter étant donné la distance temporelle et spatiale entre les deux contextes.
58. Des traces de pathologies, maladies et traumatismes ont été identifiées sur $5 \%$ des os: P. Wapnish et B. Hesse dans Stager 2008, p. 547. Dans l'Égypte antique, des animaux étaient élevés spécifiquement par rapport aux cultes animaliers: Colin - Adam - Pranjic 2014.

59. Les considérations de E. Meir (2008, p. 269-270) en faveur d'une interprétation rituelle de ces trouvailles ne sont pas pertinentes. Il est évident que "a ritual by definition is a sequence of actions performed in exactly the same manner and repeated whenever called for or on a predetermined date over a period of time", mais pour l'interprétation rituelle d'un dépôt, il faut évaluer le contexte général, la configuration chrono-stratigraphique, les modalités du dépôt, les matériels retrouvés, etc. En effet, tous les actes répétitifs, réglés et spécifiques ne sont pas nécessairement des actes rituels. 
Le chien dans la religion et dans la vie quotidienne des communautés phéniciennes et puniques

des établissements de la Méditerranée antique ${ }^{60}$, y compris dans les sites non phéniciens de la Sardaigne et de la péninsule Ibérique ${ }^{61}$. Dans les assemblages fauniques de quelques établissements phéniciens, comme Abul (VII $-\mathrm{VI}^{\mathrm{e}}$ s.; $\left.715 \mathrm{RD}\right)^{62}$, on n'a pas identifié jusque-là de Canis. Cet animal occupe donc une place systématiquement minoritaire par rapport aux espèces dominantes que sont, chez les Phéniciens, le bœuf, le mouton et, dans une moindre mesure, le porc ${ }^{63}$.

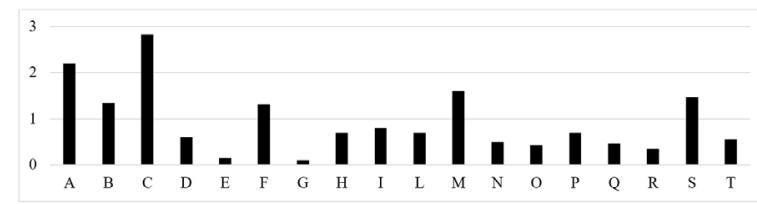

Fig. 1 - Pourcentages des restes de chien (NRD, seuls les mammifères sont pris en compte dans le calcul) dans les assemblages fauniques des sites phéniciens et puniques de Méditerranée occidentale pour lesquels au moins 300 restes de mammifères ont été déterminés (Afrique du Nord, A-D; Sardaigne, E-L; péninsule Ibérique, M-T). Les contextes religieux et funéraires ne sont pas considérés dans ce tableau parce qu'ils sont traités à part (Tab. 1).

A - Utique, IXe s. (547 RD) : Cardoso et al. 2016. B - Carthage, VIII'II (6651; dans quelques publications, les restes d'époque phénicopunique et ceux d'époque romaine ne sont pas différenciés) : Poulain 1982; Weinstock 1995; Nobis 1999; Wijngaarden-Bakker 2007; Slopsma - van Wijngaarden-Bakker - Maliepaard 2009. C - Lixus, VIII'-III S. (1621): Iborra Eres 2001; 2005; 2010. D - Ceuta, VII _ VIe s. (2240): Camarós - Estévez 2010. E - Nuraghe Sirai, VIII -VIe s. (1670): Carenti - Wilkens 2006. F - Sulky, VIII-IIle s. (757): Carenti - Wilkens 2006; B. Wilkens dans Campanella 2008, p. 249-259. G - Monte Sirai, VII $-\mathrm{VI} I^{\mathrm{e}} \mathrm{s}$. (883) : Carenti - Wilkens 2006. H - Nora, VII ${ }^{e}$ Ve s. (424) : Sorrentino 2009. I - Tharros, Ve-IVe s. (642): Farello 2000. L - Cagliari, Ve-III' s. (415): Sorrentino 1993. M - Huelva (Puerto 6, 10 et 29), IXe-IVe s. (2491): Cereijo Pecharromán - Patón Domínguez 1989; 1990; Morales Muñiz et al. 1994. N - Cerro del Villar, VIII $-\mathrm{Vl} \mathrm{l}^{\mathrm{e}}$ s. (399): Montero 1999. O - Toscanos, VIII $-\mathrm{Vl} \mathrm{I}^{\mathrm{e}} \mathrm{s}$. (3.908): Soergel 1968 ; Uerpmann - Uerpmann 1973. P - Cadix, VIIIIVIe s. (953): Estaca Gómez et al. 2015. Q - Doña Blanca, VIIIIe-IVes. (1694): Morales et al. 1994. R - Alcáçoba de Santarem, VIII'-III S. (1667): Davis 2006. S - Lisbonne, VIII-IVe s. (338): Detry - Cardoso - Bugalhão 2016. T - Castro Marim, VIII'-IVe s. (1444) : Davis 2007.

60. Par ex. K. Trantalidou dans Moore - Snyder 2006, p. 96-119 (monde grec). Dans la région gauloise, où la cynophagie est plutôt répandue pendant l'âge du Fer et jusqu'à l'époque impériale, les restes de chiens sont plus nombreux (3-4\% environ) : Horard-Herbin 2014.

61. Carenti - Wilkens 2006, p. 184 (Sardaigne); Riquelme Cantal 2001, p. 114; Iborra Eres 2004, p. 361-363; Estaca Gómez et al. 2015, p. 70-72; Pons - Colominas - Saña 2016; Catagnano 2016 (péninsule Ibérique).

62. Cardoso 2000. Seulement un reste de chien a été identifié à La Fonteta (VII ${ }^{e}-\mathrm{VI}^{\mathrm{e}}$ s. ; 443 RD) : Iborra Eres 2004, p. 288.

63. Pardo Barrionuevo 2015.
La présence de restes de chiens n'indique pas en soi qu'ils étaient consommés. Toutefois, leur consommation peut être déterminée en examinant les contextes et, surtout, la taphonomie ${ }^{64}$. Dans certains sites, des traces concernant une consommation ont été repérées sur quelques restes de chien (fig. 2-3) ${ }^{65}$. Ces ossements proviennent parfois de dépôts ou de dépotoirs contenant des restes de repas ou des déchets d'abattoir d'autres animaux. Les trouvailles de Lixus, Rocha Branca et Sulky, citées dans la note précédente, et celles d'Utique, qui seront traitées dans le prochain paragraphe, sont exemplaires à ce propos. Il faut également citer une trouvaille faite dans la zone urbaine de la ville d'Ibiza ${ }^{66}$ : dans un puits réutilisé comme dépotoir d'habitat au cours de la deuxième moitié du III S., 266 restes d'animaux (dont 204 RD) ont été mis au jour. Le chien (NRD, 73; nombre minimal d'individus, NMI, 10) est l'animal le mieux représenté, devant les Sus domesticus (NRD 80, NMI 6), Ovis/Capra $(49,6)$, Bos taurus (1) et Equus sp. $(1)^{67}$. Les chiens sont pour la plupart des adultes et toutes les parties de leur squelette sont représentées. Des brûlures liées vraisemblablement à la cuisson de la viande ont été repérées sur quelques restes; des traces de désarticulation et de prélèvement de la viande ont été identifiées sur un ulna, un fémur et un humérus. Ce contexte est très intéressant si l'on considère l'importance alimentaire du chien par rapport aux autres animaux domestiques.

64. Horard-Herbin 2014, p. 73-75.

65. À Carthage, des traces d'abattage et de consommation ont été repérées sur deux fémurs et un humérus retrouvés dans des contextes domestiques ou artisanaux d'époque punique (Weinstock 1995). À Ceuta sur un fémur, un coxale et une mandibule provenant d'un contexte domestique du VII ${ }^{\mathrm{e}} \mathrm{s}$. (fig. 3; Camarós - Estévez 2010, p. 386). À Lixus sur une scapula retrouvée dans une fosse d'époque phénicienne interprétée comme un dépotoir (Iborra Eres 2005, p. 231). À Sulky sur un humérus, un coxale et un fémur d'un chien d'un an et demi environ provenant d'une citerne réutilisée vers la fin du VI ${ }^{\mathrm{e}} \mathrm{s}$. comme un dépotoir (les restes de chien étaient de $1.5 \%$ des 314 RD; B. Wilkens dans Campanella 2008, p. 249-259). À Rocha Branca sur l'atlas et quelques vertèbres d'un chien dont d'autres portions du squelette sont représentées (ils étaient associés aux restes d'autres animaux dans un terrain placé à l'extérieur du rempart et utilisé probablement comme dépotoir; Cardoso - Gomes 1997, p. 101-104).

66. Saña 1994.

67. Sp. est l'abréviation de species: elle est utilisée pour indiquer que l'espèce dont on donne le nom de genre n'a pas été identifiée avec plus de précision, on ne peut donc pas dire s'il s'agit d'animaux domestiques ou sauvages. 


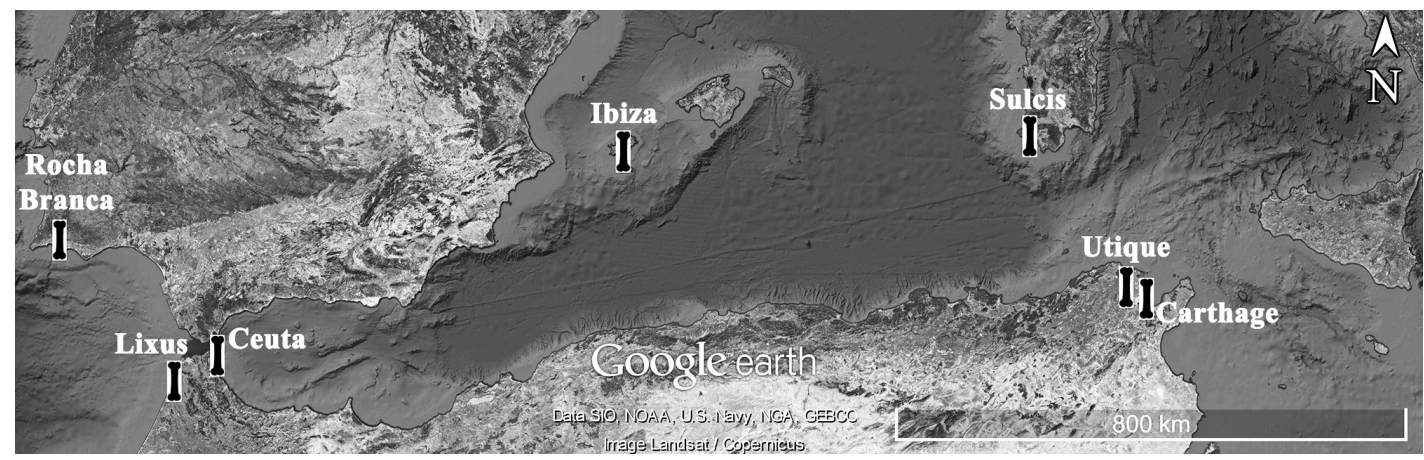

Fig. 2 - Établissements phéniciens et puniques de Méditerranée occidentale dans lesquels des traces concernant une consommation ont été repérées sur quelques restes de chien. Carte élaborée par B. D’Andrea (Google Earth @ Image Landsat).

Les données disponibles témoignent donc d'une consommation sporadique du chien dans les établissements phénico-puniques de la Méditerranée occidentale pour la période comprise entre le $\mathrm{VIII}^{\mathrm{e}} / \mathrm{VII}^{\mathrm{e}}$ et le III ${ }^{\mathrm{e}}$ s. Toutefois, cela ne confirme pas les renseignements de Justin sur la cynophagie carthaginoise (en considérant aussi qu'elle aurait perduré même après l'interdit de Darius) ni ne permet de considérer la cynophagie comme un caractère distinctif des Phéniciens/Puniques. En effet, une consommation sporadique du chien est attestée dans toute la Méditerranée, dans des sites et des contextes de chronologie et de typologie différentes ${ }^{68}$; en outre, cette pratique n'est pas prouvée, jusqu'ici, chez les Phéniciens d'Orient. Théoriquement, les Phéniciens pourraient avoir emprunté la cynophagie aux peuples indigènes de la Méditerranée occidentale (mais qui peut dire si les individus qui avaient consommé du chien dans les sites phéniciens étaient effectivement des Phéniciens?), mais la rareté des données archéozoologiques ne permet pas de vérifier cette possibilité ${ }^{69}$.

68. Par ex.: Snyder - Klippel 2003; S. Chilardi (p. 32-37) et I. Chenal-Velarde (p. 24-31) dans Moore - Snyder 2006; Horard-Herbin 2014.

69. Au Maghreb, la cynophagie est bien attestée au fil du temps et jusqu'à l'époque contemporaine (Prevost 2006), mais il n'y a pas de données "non phéniciennes " concernant l'époque préromaine. Pour la Sardaigne, également, les données relatives à l'époque préromaine sont presque absentes, alors que quelques informations sont disponibles pour la péninsule Ibérique, où la cynophagie est attestée de façon sporadique du Néolithique au début de l'époque romaine (Cardoso - Gomes 1997, p. 91-96; Liesau Esparza Arroyo - Sánchez Polo 2014; Oliver Foix 2014, p. 52; Pons - Colominas - Saña 2016; Catagnano 2016; Nieto Espinet et al. 2017).

\section{LES CONTEXTES RITUELS}

Des restes d'animaux retrouvés dans une nécropole ou dans un sanctuaire ne sont pas nécessairement le résultat d'un rituel; au contraire, des restes d'animaux retrouvés dans des contextes domestiques, artisanaux, etc. peuvent être le résultat d'une pratique rituelle (on peut citer l'exemple des dépôts de fondation, qui sont très répandus dans les sites phénico-puniques) ${ }^{70}$.

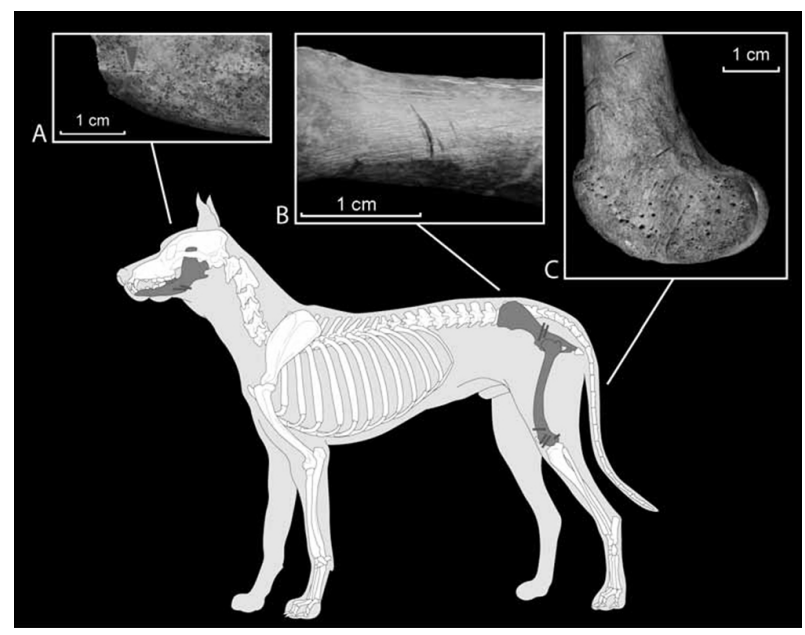

Fig. 3 - Ceuta : restes de chien avec des traces d'abattage et de consommation retrouvés d'un contexte domestique du VII $\mathrm{s}$. (Camarós - Estévez 2010, p. 387, fig. 8).

70. Plusieurs travaux sur l'archéologie du rite proposent un approfondissement méthodologique, théorique et pratique de ces questions: par ex. Ekroth - Wallenstein 2013. 
Le chien dans la religion et dans la vie quotidienne des communautés phéniciennes et puniques

194 Bruno D'AndReA

Un autre aspect important concerne le type de rituel dans lequel les animaux sont impliqués, selon la modalité d'exécution (animal entier ou partagé, brûlé ou non brûlé, etc.) et les objectifs (expiation, offrande votive, etc.). En particulier, il est nécessaire de différencier les sacrifices de type alimentaire - comportant une consommation de la viande, laquelle est généralement partagée entre l(es) homme(s) et le(s) dieu(x) - des sacrifices non alimentaires dans lesquels, par exemple, la victime est entièrement brulée pour la divinité (comme dans l'holocauste hébraïque ${ }^{71}$ ) ou bien utilisée dans des rites apotropaïques ou prophylactiques. Quant aux contextes funéraires, les restes d'animaux peuvent être liés aux pratiques sacrificielles et/ou aux croyances eschatologiques: nourriture du mort pour sa vie dans l'au-delà ${ }^{72}$, offrande aux dieux, sacrifice apotropaïque ou prophylactique, banquet funéraire, mort d'accompagnement, etc. ${ }^{73}$. Une dernière problématique concerne le type de chien utilisé dans les pratiques rituelles: l'âge, la couleur, la taille, etc. (malheureusement, ces données ne sont pas toujours perceptibles par l'archéologie).

Le Tab. 1 ressemble les contextes dans lesquels des chiens ont été retrouvés et pour lesquels une interprétation rituelle a été proposée, en assignant un indice de fiabilité à chaque contexte (fig. 4). Dans certains cas, des dépôts vraisemblablement rituels ne sont pas associés avec certitude à des complexes religieux. Dans les fouilles menées à Utique au cours des dernières années par une équipe tuniso-espagnole, beaucoup de céramique de table et plus de 500 restes d'animaux ont été mis au jour dans un puits (Tab. 1, nº 4 ). Ces restes avaient été déposés dans le puits au moment de son oblitération (suite, probablement, à sa salinisation) vers le troisième quart du $\mathrm{IX}^{\mathrm{e}} \mathrm{s}$. Leur dépôt avait eu lieu en très peu de temps, ce qui fait penser qu'il

71. L'existence de l'holocauste dans le monde phénico-punique a été supposée sur la base des "tarifs" (note 29) et d'une inscription d'époque tardo-punique $($ KAI $159=$ Jongeling 2008, Hr. Medeine N1), mais elle n'est pas prouvée.

72. La croyance en une vie corporelle post mortem et d'une survivance de l'âme n'est pas attestée avec certitude chez les Phéniciens (Ribichini 2004b); toutefois, la présence dans les tombes d'aliments est bien attestée et, dans la plupart de cas, elle semble associée au défunt (BénichouSafar 1982, p. 263-266).

73. Pour les sacrifices phénico-puniques: Lipiński 1995 , p. 463-482. Cf. D'Andrea (à paraître). s'agissait des restes d'un grand repas dans lequel au moins (NMI) 10 Bos taurus (NRD 265), 6 Sus sp. (88) et 4 Ovis/Capra (142) avaient été consommés. Des restes de Equus caballus (NMI 4, NRD 26), Canis familiaris (3, 12), Loxodonta africana (1) et Testudo $s p$. (1) faisaient partie du même assemblage. Des traces de désarticulation, découpe et prélèvement de la viande ont été identifiées sur un talon et un tibia; elles témoignent que les chiens avaient été consommés. La thèse selon laquelle il s'agissait d'un dépôt rituel lié à l'oblitération du puits et à la célébration d'un banquet sacrificiel doit être prise en compte, mais elle ne peut pas être vérifiée: il nous manque pour cela des données concernant sans doute la sphère religieuse et une interprétation fonctionnelle du complexe dont le puits fait partie $^{74}$.

Dans le site (phénicien?) de Quinta do Almaraz, les squelettes plus ou moins entiers de 13 (NMI) chiens ont été retrouvés dans un fossé placé originairement (VIII ${ }^{\mathrm{e}}$ s.) à l'extérieur des remparts $\left(\mathrm{n}^{\circ} 14\right)$. Au cours du VII ${ }^{\mathrm{e}}$ s., le fossé avait été englobé dans le rempart et, dans un bref délai, il fut rempli et apparemment réutilisé comme dépotoir (un quartier d'habitat se trouvait à proximité): l'assemblage faunique est composé de restes de Canis familiaris, Ovis/Capra, Sus sp., Rattus, Meles meles (blaireau), oiseaux et poissons. Malheureusement, on ne saurait dire avec certitude si les chiens avaient été déposés au même moment ou à des moments différents (certaines parties du fossé ont été utilisées jusqu'au IVe s.). Dans aucun des cas les chiens n'ont fait l'objet de consommation. L'absence quasi-totale de leurs crânes et de traces liées à leur découpe indique que les crânes avaient été prélevés quelque temps après la mort de $\mathrm{l}^{\prime}$ animal ${ }^{75}$. Cette action pourrait avoir une signification rituelle (avec la réutilisation des crânes), mais en l'absence de données de compa-

74. Mais une interprétation rituelle du remplissage du puits ne peut pas être écartée: selon les archéozoologues qui ont étudié le complexe, la faune retrouvée dans le puits «does not suggest that the source of the remains is ritual sacrifice, or even less a sanctuary deposit" (Cardoso et al. 2016, p. 321). Cette thèse, fondée principalement sur la présence importante du cochon, repose sur un a priori - celui de l'interdit rituel du cochon - qui en réalité est loin d'être prouvé.

75. Au moins un an et demi plus tard: Correia 2015, p. 120. 
raison, il est impossible de vérifier cette thèse. On peut toutefois noter que dans quelques contextes funéraires phénico-puniques, seuls des crânes de chiens ont été retrouvés (à Sidon, comme nous l'avons vu précédemment, et à Carthage: $\mathrm{n}^{0}$ 6-7).

TAB. 1 - Contextes Rituels (ou interprétés Comme tels) Des Sites Phéniciens et Puniques de MéditerRanée occidentale dans lesquels des restes de chiens ont été Retrouvés (Malte, 1-3; Afrique du Nord, 4-7; Sardaigne, 8-9; Sicile, 10-13; PÉNINSUle IBÉRIQUe, 14-24).

\begin{tabular}{|c|c|c|c|c|}
\hline $\mathrm{N}^{\circ}$ & $\begin{array}{l}\text { Site et } \\
\text { contexte }\end{array}$ & Chronologie & Description et "fiabilité » des contextes* & Bibliographie \\
\hline 1 & $\begin{array}{l}\text { Malte } \\
\text { (sanctuaire de } \\
\text { Tas-Sil囚) }\end{array}$ & VII ${ }^{\mathrm{e}}-\mathrm{I}^{\mathrm{er}} \mathrm{s}$. & $\begin{array}{l}\text { Des marques de dents de chiens ont } \\
\text { été identifiées sur des restes d'animaux } \\
d^{\prime} \text { «époque punique»; une dent de } \\
\text { chien faisait partie de l'assemblage fau- } \\
\text { nique des } \mathrm{II}^{\mathrm{e}}-\mathrm{I}^{\mathrm{er}} \text { s. }(\mathrm{X})\end{array}$ & $\begin{array}{l}\text { Corrado - Bonanno - Vella } \\
2002 \text {; } \\
\text { De Grossi Mazzorin - } \\
\text { Battafarano } 2012\end{array}$ \\
\hline 2 & $\begin{array}{l}\text { Malte } \\
\text { (nécropole de } \\
\text { Mtarfa) }\end{array}$ & $?$ & $\begin{array}{l}\text { Les restes d'un chien ont été retrouvés, } \\
\text { avec les restes d'un capriné, dans une } \\
\text { tombe à chambre (sépulture collective) } \\
(\mathrm{XX})\end{array}$ & Sagona 2002, p. 881 \\
\hline 3 & $\begin{array}{l}\text { Malte } \\
\text { (nécropole de } \\
\text { Rabat) }\end{array}$ & $?$ & $\begin{array}{l}\text { Des portions de squelettes de deux } \\
\text { chiens ont été retrouvées, avec des } \\
\text { tessons de céramique et un objet en } \\
\text { bronze, dans la chambre funéraire } \\
\text { d'une tombe qui contenait trois indivi- } \\
\text { dus }(\mathrm{XX})\end{array}$ & Sagona 2002, p. 966 \\
\hline 4 & $\begin{array}{l}\text { Utique } \\
\text { ( "complexe archi- } \\
\text { tectural») }\end{array}$ & $\mathrm{IX}^{\mathrm{e}} \mathrm{s}$ & $\begin{array}{l}\text { Les restes de plusieurs chiens (dont } \\
\text { certains présentaient des traces de } \\
\text { découpe) ont été retrouvés dans le } \\
\text { remplissage d'un puits avec des restes } \\
\text { d'autres animaux et des tessons de céra- } \\
\text { mique }(\mathrm{XX})\end{array}$ & Cardoso et al. 2016 \\
\hline 5 & $\begin{array}{l}\text { Carthage } \\
\text { (nécropole) }\end{array}$ & $V I^{e}-V^{e} s$ & $\begin{array}{l}\text { Les squelettes complets d'un chien } \\
\text { adulte et d'un jeune chiot ont été } \\
\text { retrouvés, avec des restes d'autres } \\
\text { animaux et quelques objets, dans une } \\
\text { niche extérieure à un hypogée (XXX) }\end{array}$ & $\begin{array}{l}\text { Gaillard 1942, p. 331-333; } \\
\text { Bénichou-Safar 1982, p. 279- } \\
280\end{array}$ \\
\hline 6 & $\begin{array}{l}\text { Carthage } \\
\text { (nécropole) }\end{array}$ & $\mathrm{V}^{\mathrm{e}}-\mathrm{II}^{\mathrm{e}} \mathrm{s}$ & $\begin{array}{l}\text { Les restes de plusieurs chiens (au moins } \\
\text { sept) ont été retrouvés, avec des restes } \\
\text { d'autres animaux, dans une chambre } \\
\text { funéraire qui contenait une quarantaine } \\
\text { de squelettes humains. Les restes d'ani- } \\
\text { maux étaient mêlés aux restes humains } \\
(\mathrm{XXX})\end{array}$ & $\begin{array}{l}\text { Delattre 1905, } \\
\text { p. 27-29 }\end{array}$ \\
\hline 7 & $\begin{array}{l}\text { Carthage } \\
\text { (nécropole) }\end{array}$ & $\mathrm{IV}^{\mathrm{e}}-\mathrm{III}^{\mathrm{e}} \mathrm{s}$ & $\begin{array}{l}\text { Le crâne d'un chien a été retrouvé de- } \\
\text { vant la porte } \\
\text { d'une chambre funéraire (XXX) }\end{array}$ & Delattre 1901, p. 18 \\
\hline 8 & $\begin{array}{l}\text { Villamar } \\
\text { (nécropole) }\end{array}$ & $\mathrm{IV}^{\mathrm{e}}-\mathrm{II}^{\mathrm{e}} \mathrm{s}$ & $\begin{array}{l}\text { Les squelettes (entiers?) de deux chiens } \\
\text { ont été retrouvés dans une chambre } \\
\text { funéraire (XXX) }\end{array}$ & $\begin{array}{l}\text { Communication personnelle } \\
\text { de } \\
\text { E. Pompianu }\end{array}$ \\
\hline 9 & $\begin{array}{l}\text { Sulky } \\
\text { (nécropole) }\end{array}$ & $I^{\mathrm{e}}-\mathrm{I}^{\mathrm{er}} \mathrm{s}$ & $\begin{array}{l}\text { La mandibule brûlée d'un chien a été } \\
\text { retrouvée à côté de restes humains } \\
\text { incinérés dans une chambre funéraire } \\
\text { (sépulture collective) (XXX) }\end{array}$ & Guirguis - Unali 2012 \\
\hline
\end{tabular}


Le chien dans la religion et dans la vie quotidienne des communautés phéniciennes et puniques

196 Bruno D'AndreA

\begin{tabular}{|c|c|c|c|c|}
\hline 10 & $\begin{array}{l}\text { Motyé } \\
\text { (aire sacrée du } \\
\text { «Kothon») }\end{array}$ & $\mathrm{VIII}^{\mathrm{e}}-\mathrm{V}^{\mathrm{e}} \mathrm{s}$ & $\begin{array}{l}\text { Les restes d'un chien (de plus de huit } \\
\text { mois) faisaient partie du remplissage } \\
\text { d'une favissa avec des restes d'autres } \\
\text { animaux, de nombreux coquillages, des } \\
\text { tessons de céramique et quelques objets } \\
\text { (XXX) }\end{array}$ & $\begin{array}{l}\text { F. Alhaique dans Kothon } \\
2012 \text {, p. 8-33 }\end{array}$ \\
\hline 11 & $\begin{array}{l}\text { Motyé } \\
\text { (aire sacrée du } \\
\text { "Kothon») }\end{array}$ & $I^{\mathrm{e}} \mathrm{s}$ & $\begin{array}{l}\text { Quelques restes de chiens faisaient par- } \\
\text { tie de l'assemblage faunique retrouvé } \\
\text { dans le temple C2 et dans le sanctuaire } \\
\text { C3 (X) }\end{array}$ & $\begin{array}{l}\text { F. Alhaique dans Mozia XI, } \\
\text { p. 521-532 }\end{array}$ \\
\hline 12 & $\begin{array}{l}\text { Motyé } \\
\text { (sanctuaire } \\
\text { «tophet») }\end{array}$ & $\begin{array}{l}\text { VIII' }^{\text {e- }} \\
\text { IV }^{\mathrm{e}} \mathrm{s}\end{array}$ & $\begin{array}{l}\text { Quelques restes de chiens (avec des } \\
\text { traces de découpe?) faisaient partie de } \\
\text { l'assemblage faunique retrouvé dans le } \\
\text { sanctuaire }(X)\end{array}$ & $\begin{array}{l}\text { C. Di Patti dans } \\
\text { Ciasca et al. } 1996, \\
\text { p. } 329\end{array}$ \\
\hline 13 & $\begin{array}{l}\text { Solonte } \\
\text { (nécropole) }\end{array}$ & III $^{\mathrm{e}} \mathrm{s}$. & $\begin{array}{l}\text { Une dent de chien a été retrouvée à } \\
\text { côté des restesi ncinérés du (des) dé- } \\
\text { funt }(\mathrm{s})(\mathrm{X})\end{array}$ & $\begin{array}{l}\text { Calascibetta } 2009, \\
\text { p. } 636\end{array}$ \\
\hline 14 & $\begin{array}{l}\text { Quinta do Almaraz } \\
\text { (fossé du rempart) }\end{array}$ & $\begin{array}{l}\text { VIII }^{\mathrm{e}}- \\
\text { IV }^{\mathrm{e}} \text { s. ? }\end{array}$ & $\begin{array}{l}\text { Des squelettes complets de chiens ont } \\
\text { été retrouvés dans un fossé placé à l'ex- } \\
\text { térieur du rempart }(\mathrm{X})\end{array}$ & $\begin{array}{l}\text { Cardoso - Gomes 1997, } \\
\text { p. 107; } \\
\text { Correia } 2015\end{array}$ \\
\hline 15 & $\begin{array}{l}\text { Huelva } \\
\text { (nécropole) }\end{array}$ & $\mathrm{VIII}^{\mathrm{e}}-\mathrm{VI}^{\mathrm{e}} \mathrm{s}$. & $\begin{array}{l}\text { Deux squelettes de chiens ont été re- } \\
\text { trouvés, avec des tessons de céramique, } \\
\text { au-dessus d'une tombe à fosse (XXX) }\end{array}$ & $\begin{array}{l}\text { Niveau de Villedary } 2008, \\
\text { p. 109-110 }\end{array}$ \\
\hline 16 & $\begin{array}{l}\text { Alcácer do Sal } \\
\text { (nécropole) }\end{array}$ & $\mathrm{VIII}^{\mathrm{e}}-\mathrm{IV}^{\mathrm{e}} \mathrm{s}$ & $\begin{array}{l}\text { Quelques restes de chiens ont été re- } \\
\text { trouvés dans la nécropole }(\mathrm{X})\end{array}$ & Arruda - Cardoso 2016 \\
\hline 17 & $\begin{array}{l}\text { Ibiza } \\
\text { (nécropole) }\end{array}$ & $\begin{array}{l}\mathrm{VII}^{\mathrm{e}}- \\
\mathrm{III}^{\mathrm{e}} \mathrm{s} . ?\end{array}$ & $\begin{array}{l}\text { Plusieurs squelettes entiers de chiens } \\
\text { ont été retrouvés dans les chambres } \\
\text { funéraires de la nécropole } \\
(\mathrm{XXX})\end{array}$ & $\begin{array}{l}\text { Morales Pérez 2008, p. 24; } \\
\text { Fernández et al.2015, p. 136- } \\
137\end{array}$ \\
\hline 18 & $\begin{array}{l}\text { Cerro de la Tortuga } \\
\text { (sanctuaire?) }\end{array}$ & $\begin{array}{l}\mathrm{VII}^{\mathrm{e}}- \\
\mathrm{VI}^{\mathrm{e}} \mathrm{s} . ?\end{array}$ & $\begin{array}{l}\text { Le squelette d'un chien a été retrou- } \\
\text { vé, avec des tessons de céramique et } \\
\text { quelques objets, dans une citernedu } \\
\text { "sanctuaire" }(\mathrm{X})\end{array}$ & $\begin{array}{l}\text { Niveau de Villedary } 2008, \\
\text { p. } 110\end{array}$ \\
\hline 19 & $\begin{array}{l}\text { Cerro de la Tortuga } \\
\text { (nécropole) }\end{array}$ & $\mathrm{VI}^{\mathrm{e}}-\mathrm{III}^{\mathrm{e}} \mathrm{s}$ & $\begin{array}{l}\text { Plusieurs restes de chiens ( } 4 \% \text { environ) } \\
\text { faisaient partie de l'assemblage faunique } \\
\text { retrouvé dans la nécropole (XX) }\end{array}$ & $\begin{array}{l}\text { Uerpmann - Uerpmann } \\
\text { 1973, p. 68-82 }\end{array}$ \\
\hline 20 & $\begin{array}{l}\text { Malaga } \\
\text { (nécropole) }\end{array}$ & $V^{\mathrm{e}}-\mathrm{IV}^{\mathrm{e}} \mathrm{s}$ & $\begin{array}{l}\text { Le squelette incinéré d'un chien (de } \\
\text { plus d'un an) a été retrouvé avec des } \\
\text { restes d'autres animaux, des tessons } \\
\text { de céramique et divers objets dans une } \\
\text { couche de cendres, devant la porte } \\
\text { d'une chambre funéraire. Le chien } \\
\text { avait été tué et n'avait pas fait l'objet de } \\
\text { consommation (XXX) }\end{array}$ & $\begin{array}{l}\text { M. Montero dans Martín } \\
\text { Ruiz - Pérez-Malumbres } \\
\text { Landa - García Carretero } \\
2005\end{array}$ \\
\hline 21 & $\begin{array}{l}\text { Cadix } \\
\text { (nécropole) }\end{array}$ & $\mathrm{V}^{\mathrm{e}}-\mathrm{II}^{\mathrm{e}} \mathrm{s}$ & $\begin{array}{l}\text { Les squelettes et les portions de sque- } \\
\text { lettes de plusieurs chiens ont été } \\
\text { retrouvés, avec des restes d'autres } \\
\text { animaux, des tessons de céramique et } \\
\text { divers objets, dans des puits, des fosses } \\
\text { et d'autres structures de la nécropole } \\
\text { punique (mais généralement ils ne sont } \\
\text { pas associés aux tombes de la nécro- } \\
\text { pole) (XXX) }\end{array}$ & $\begin{array}{l}\text { Ferrer Albelda - Niveau de } \\
\text { Villedary 2004; Niveau de } \\
\text { Villedary } 2008\end{array}$ \\
\hline 22 & $\begin{array}{l}\text { Villaricos } \\
\text { (nécropole) }\end{array}$ & $\mathrm{V}^{\mathrm{e}}-\mathrm{II}^{\mathrm{e}} \mathrm{s}$ & $\begin{array}{l}\text { Deux restes de chiens ont été retrouvés } \\
\text { dansune chambre funéraire }(X)\end{array}$ & Castaños Ugarte 1994 \\
\hline
\end{tabular}




\begin{tabular}{|c|c|c|c|c|}
\hline 23 & $\begin{array}{l}\text { Garvão } \\
\text { (sanctuaire?) }\end{array}$ & $\mathrm{IV}^{\mathrm{e}}-\mathrm{III}^{\mathrm{e}} \mathrm{s}$ & $\begin{array}{l}\text { Les restes de plusieurs chiens ont été re- } \\
\text { trouvés dans le remplissage d'une fosse } \\
\text { qui contenait aussi des restes d'autres } \\
\text { animaux, des tessons de céramique et } \\
\text { divers objets votifs (XX) }\end{array}$ & $\begin{array}{l}\text { Gomes - Tavares } \\
\text { da Silva 1994; } \\
\text { Cardoso - Gomez 1997, } \\
\text { p. 105-107 }\end{array}$ \\
\hline 24 & $\begin{array}{l}\text { Ibiza } \\
\text { (grotte-sanctuaire } \\
\text { d'Es Culleram) }\end{array}$ & $\mathrm{IV}^{\mathrm{e}}-\mathrm{II}^{\mathrm{e}} \mathrm{s}$ & $\begin{array}{l}\text { Des marques de dents de chiens ont été } \\
\text { identifiées sur quelques restes d'ani- } \\
\text { maux retrouvés dans la grotte (mais } \\
\text { dans l'assemblage faunique il n'y avait } \\
\text { pas de chiens) }(\mathrm{X})\end{array}$ & Morales Pérez 2003 \\
\hline
\end{tabular}

* Indice de fiabilité déterminé en fonction de la fonction rituelle des contextes, de l'interprétation rituelle des restes de chien et de la "qualité » des fouilles et des publications (X: faible; XX: moyenne; XXX: bonne).
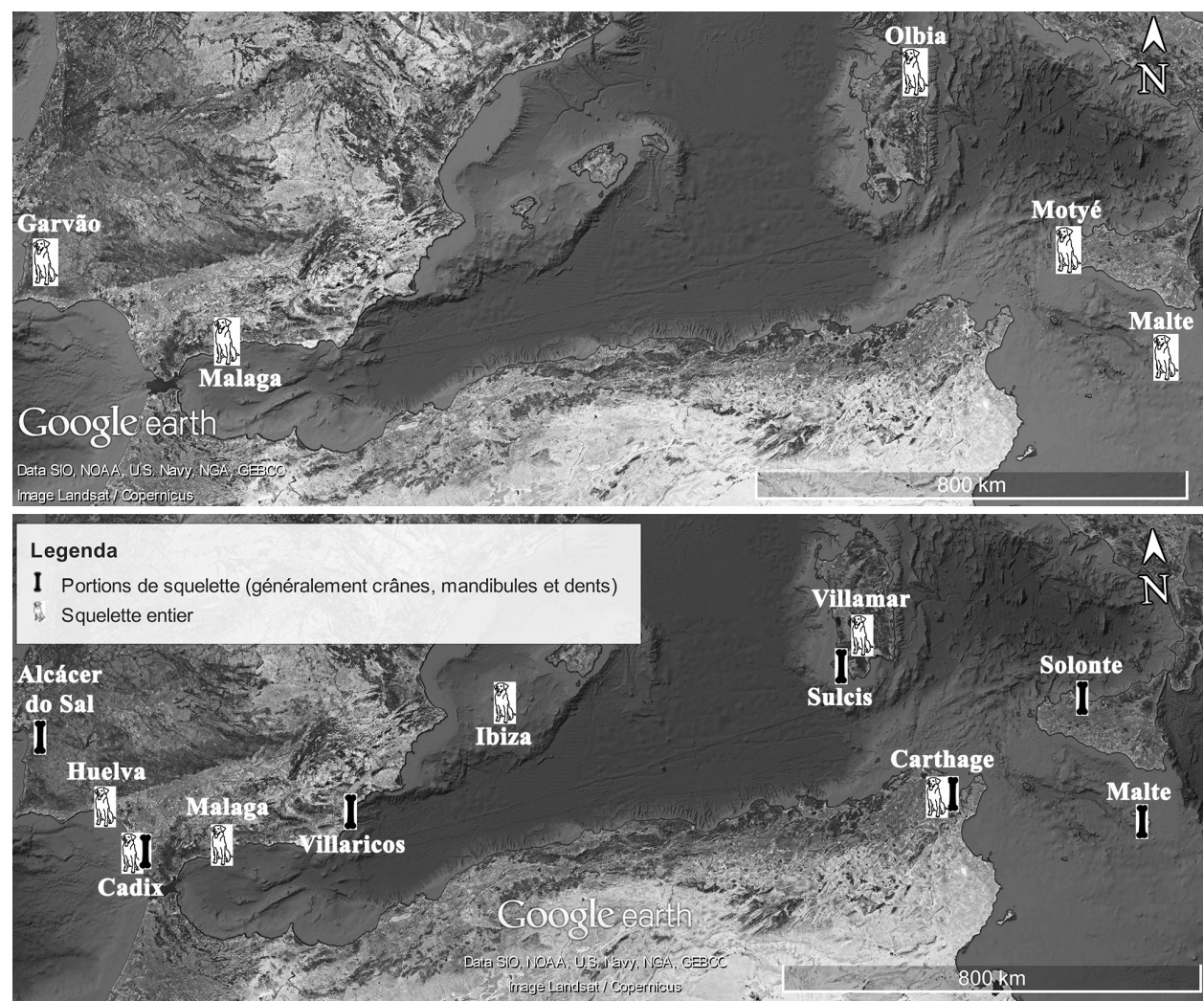

Fig. 4 - Établissements phéniciens et puniques de Méditerranée occidentale où des chiens ont été retrouvés dans des sanctuaires (en haut) ou dans des nécropoles (en bas). Carte élaborée par B. D’Andrea (Google Earth (c) Image Landsat).

\section{Les sanctuaires}

Dans les sites de Cerro de la Tortuga et de Garvão l'interprétation comme aires sacrées des complexes dans lesquels les restes de chiens ont été découverts reste débattue, de même que le caractère phénicien/punique des deux sites. Au château de Garvão, dans les années 1980, M. Beirão a fouillé une fosse dans laquelle ont été déposés au cours de la seconde moitié du III ${ }^{\mathrm{e}} \mathrm{s}$. beaucoup de céramique, différents objets votifs et, au fond, plusieurs restes d'animaux $\left(\mathrm{n}^{\circ} 23\right) .15 \%$ des RD appartiennent à des chiens, auxquels des Ovis/Capra (36\%), des Bos (23\%) et des Sus (18\%) sont associés. Cette fosse a été interprétée comme un bothros qui devait être associé à un sanctuaire (non identifié). L'interprétation rituelle de la fosse est confirmée par la découverte d'objets ayant une fonction votive et religieuse, comme des plaquettes figurant des yeux, un maxillaire humain et un personnage féminin probable- 
Le chien dans la religion et dans la vie quotidienne des communautés phéniciennes et puniques

ment divin. Les restes d'animaux pourraient être liés aux rites exécutés au moment du creusement et du remplissage de la fosse ou, moins probablement, aux sacrifices pratiqués dans le sanctuaire; ils pourraient témoigner de sacrifices partagés (à des fins votives?). Sur les restes des chiens, il n'y a pas de traces de consommation. Cependant, des traces de traumatisme indiquant une violence suggèrent peut-être le meurtre de ces animaux. Au fond de la fosse, à peu près au même niveau que là où l'on a retrouvé les restes d'animaux, a été découvert le crâne d'une femme de 35-40 ans, dont la mort a été causée par trois coups portés à l'occipital par un objet contondant ${ }^{76}$. Ce crâne, qui a été interprété comme un dépôt de fondation, avait très probablement fait l'objet d'un rituel.

Entre la fin des années 1950 et le début des années 1960, au cours des fouilles menées par J.M. Muñoz Gambero dans le sanctuaire de Cerro de la Tortuga, le squelette d'un chien et des tessons de céramique ont été retrouvés dans une citerne $\left(\mathrm{n}^{\circ} 18\right)$. L'absence de données plus précises concernant le contexte (et sa chronologie) ne permet pas de proposer une interprétation rituelle de ce dépôt, d'autant plus que c'est l'interprétation même du complexe comme sanctuaire (ainsi que son attribution aux Phéniciens) qui reste débattue en raison de la médiocre qualité des fouilles et des publications ${ }^{77}$.

En général, dans les aires sacrées phénico-puniques, la découverte de restes de chien est plutôt rare, mais il faut considérer que nous ne connaissons pas beaucoup de sanctuaires et, surtout, que pour la plupart d'entre eux, il n'y a pas eu d'étude archéozoologique. Au complexe religieux du «Kothon» de Motyé, daté du VIII ${ }^{e}$ au IV ${ }^{e}$ s. et fouillé par une équipe de l'Université de Rome « $L a$ Sapienza» dirigée par L. Nigro, deux restes de chien ont été repérés dans deux US concernant, respectivement, la destruction et la spoliation du temple C2 $\left(V^{e}\right.$ s.) et du sanctuaire C3, lequel se superpose au temple $\mathrm{C} 2$ au début du IVe $\mathrm{s}$. (n 11$)^{78}$. Les contextes

76. Gomes - Tavares da Silva 1994, p. 39

77. Muñoz Gambero 2009.

78. F. Alhaique dans Mozia XI, p. 521-532 (les autres restes de chien cités dans le catalogue proviennent de couches superficielles). Il est intéressant de noter qu'aucun reste de Canis n'a été identifié dans les fouilles des autres secteurs (Mozia X-XIII). Je remercie L. Nigro de m'avoir confié, dans le cadre d'un projet soutenu par le LabEx ARCHIMEDE (note 105), l'étude de l'assemblage faunique du sanctuaire C7, qui fait partie du complexe du Kothon. de provenance ne permettent pas d'accorder à ces restes une fonction rituelle. En revanche, on peut proposer une interprétation rituelle pour les restes d'un chien de plus de huit mois mis au jour dans le remplissage d'une favissa (F.2950) placée dans le même complexe religieux ${ }^{79}$. Cette favissa avait été utilisée plusieurs fois du VII ${ }^{\mathrm{e}}$ au second quart du $\mathrm{VI}^{\mathrm{e}}$ s. et elle avait été fermée par un rituel d'oblitération vers la moitié du VI ${ }^{\mathrm{e}} \mathrm{s} .\left(\mathrm{n}^{\circ} 10\right)$. Dans son remplissage, ont été retrouvés plusieurs tessons de céramique (dont l'un porte une inscription dédicatoire en grec), divers objets et un assemblage faunique caractérisé par de nombreux coquillages, ainsi que par des restes de Ovis/Capra (NMI 3), Sus domesticus (2), Bos taurus (1), Canis familiaris (1) et d'un oiseau de grande taille. Cet assemblage a été interprété comme une offrande alimentaire ${ }^{80}$.

Quelques restes de Canis ont été mis au jour au tophet de Motyé (no 12). C. Di Patti, qui a étudié ce complexe faunique, dit que les restes de chien, comme ceux de Bos taurus, Cervus, Equus et Sus, n'étaient pas contenus dans des urnes cinéraires (qui, dans les tophets, contiennent généralement des restes calcinés d'enfants et/ou d'agneaux/ chevreaux). Selon elle, ces restes appartiennent à des individus adultes et ont des "rotture così nette da far pensare a resti di macellazione ${ }^{81}$. J. I. s. Whitaker, qui fut le premier à fouiller le tophet de Motyé en 1919, avait signalé la présence de restes de chien dans des urnes cinéraires, mais cette affirmation n'a pas été confirmée par les études de C. Di Patti ${ }^{82}$. Une remarque semblable peut être faite pour le tophet de Carthage ${ }^{83}$. Aucun reste de chien n'a été retrouvé dans les autres tophets pour lesquels des études de la faune sont disponibles ${ }^{84}$.

Un seul reste de chien (une dent) a été retrouvé sur plus de $2000 \mathrm{RD}$ mis au jour dans le «bassin» 52 du sanctuaire maltais de Tas-Silg, qui a été utilisé à partir du $\mathrm{III}^{\mathrm{e}}$ millénaire et réaménagé par les Phéniciens au cours du VIII s. (durant cette période, il était consacré à Astarté; $\mathrm{n}^{0} 1$ ).

79. F. Alhaique dans Kothon 2012, p. 33. Voir, dans le même ouvrage, L. Nigro, p. 8-12.

80. F. Alhaique dans Kothon 2012, p. 33.

81. C. Di Patti dans Ciasca et al. 1996, p. 329.

82. Whitaker 1921, p. 257. Cf. Ciasca et al. 1996.

83. D'Andrea (à paraître), note 12 .

84. Voir D’Andrea (à paraître). 
Ce bassin avait été rempli au cours du $\mathrm{I}^{\mathrm{er}} \mathrm{s}$., après l'occupation romaine de Malte en 218; la dent a été considérée comme intrusive. Les études de la faune d' "époque punique" récupérée lors des fouilles pratiquées par l'Université de Malte au cours des années 1990 ont identifiés exclusivement des Ovis/Capra (96\% des RD) et des Bos taurus $(4 \%)^{85}$. En revanche, les traces de rognage et de morsure identifiées sur quelques os indiquent que des chiens avaient eu accès à ces restes après leur dépôt ou au moment de cérémonies sacrificielles (par exemple au cours des banquets rituels). Le même constat a été fait à propos du sanctuaire de Kition-Bamboula (4\% environ des ossements portent des marques de dents) ${ }^{86}$, du sanctuaire de la grotte d'Es Culleram à Ibiza $\left(\mathrm{IV}^{\mathrm{e}}-\mathrm{II}^{\mathrm{e}} \mathrm{s}\right.$.; $\left.\mathrm{n}^{\mathrm{o}} 24\right)$ et de celui du lac de Vénus à Pantelleria (fréquenté de l'époque punique à l'époque romaine $)^{87}$.

Ces données posent la question de l'accès des chiens aux aires sacrées. Un texte hittite du $\mathrm{XIV}^{\text {e s. }}$ contenant des instructions aux prêtres et aux officiels d'un temple prescrit de ne pas laisser entrer les porcs et les chiens dans le sanctuaire ${ }^{88}$. On retrouve une prescription semblable dans un manuscrit de Qumrân dans lequel il est précisé que des chiens auraient pu manger les os déposés dans le sanctuaire quand il y avait encore de la viande ${ }^{89}$. Une interdiction aux chiens est attestée aussi dans quelques sanctuaires grecs et romains ${ }^{90}$. Si certains sanctuaires excluaient les chiens, d'autres au contraire en abritaient: dans quelques sanctuaires gréco-romains (ainsi que dans le monde mésopotamien), les chiens étaient présents soit en tant que gardiens fidèles, soit comme chiens sacrés ou encore en jouant un rôle actif dans les rites thérapeutiques ${ }^{91}$. Les seules informations à disposition pour le monde phénico-punique sont celles susmentionnées, qui ne permettent pas de faire de généralisations. La question de l'accès des chiens aux sanctuaires, et donc aux restes des sacrifices, est directement liée à l'existence de précautions

85. Corrado - Bonanno - Vella 2002.

86. Gardeisen - Garcia Petit - Piquès 2015, p. 360. Voir la note 42.

87. Wilkens 2006

88. Collins 1992, p. 6; Sumaka'i Fink 2003, p. 49 (KUB 13.4).

89. Sumaka'i Fink 2003, p. 50. Cf. Schattner-Rieser 2009, p. 300-301.

90. Plutarque, $Q R, 111$; Pline, NH, 10, 79. Cf. Georgoudi 2015.

91. Élien, $N A, 11$, 3. Voir la note 22 et Georgoudi 2015. visant à l'éviter: des enclos ou des limites naturelles, un personnel préposé à la surveillance des entrées au sanctuaire, la déposition des restes des sacrifices dans des lieux non accessibles aux carnivores, etc.

\section{Les nécropoles}

Des données plus nombreuses proviennent des contextes funéraires. Un bon nombre de chiens ont été retrouvés dans des puits et d'autres structures remontant aux $\mathrm{V}^{\mathrm{e}}-\mathrm{II}^{\mathrm{e}} \mathrm{s}$. (pour la plupart aux III $-\mathrm{II}^{\mathrm{e}}$ s.) et fouillées dans la nécropole de Cadix ( $\mathrm{n}^{\circ}$ 21). Généralement, ces structures n'étaient pas liées directement aux sépultures. Dans six cas, les squelettes entiers et en connexion anatomique d'un à quatre chiens étaient déposés au fond d'un puits rempli avec de la terre vierge, des tessons de céramique et, parfois, des restes d'autres animaux (fig. 5). Dans deux de ces puits, les chiens étaient associés à un crâne humain, qui, dans un cas, portait des traces de mort violente. A.M. Niveau de Villedary propose d'interpréter ces puits « como un espacio sacro en el que tienen lugar diversas actividades litúrgicas en las que los sacrificios y ofrendas de perros se convierten en el rito central. Rituales con connotaciones ctónicas y funerarias... $»^{92}$.

Dans trois autres puits et dans une fosse, les restes de chiens n'étaient ni complets ni en connexion anatomique; dans ces cas, ils étaient généralement associés à des tessons de céramique et à des restes d'autres mammifères, de poissons, de mollusques et de coquillages. Dans un premier temps, Niveau de V. a supposé qu'il pouvait s'agir des restes des banquets funéraires qui avaient eu lieu dans la nécropole ${ }^{93}$. Plus tard, s'appuyant surtout sur le fait que les chiens sont représentés généralement par des portions de squelette spécifiques (et symboliques) comme le crâne et la mandibule, elle a proposé une interprétation semblable à celle avancée pour les squelettes entiers ${ }^{94}$.

92. Niveau de Villedary 2008, p. 128. Auparavant, elle avait proposé que les chiens constituaient des sacrifices de fondation et de sacralisation des puits (ensuite utilisés comme des «basureros sacros") : Ferrer Albelda - Niveau de Villedary 2004.

93. Ferrer Albelda - Niveau de V. 2004.

94. Niveau de V. 2008, p. 128-129. 
Le chien dans la religion et dans la vie quotidienne des communautés phéniciennes et puniques

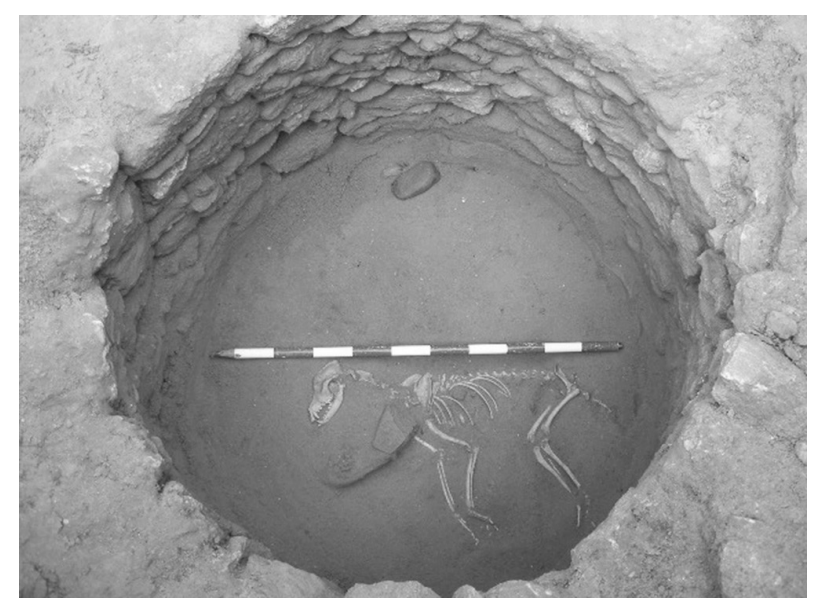

Fig. 5 - Cadix, "Ciudad de la Justicia » (III ${ }^{\mathrm{e}}$ s.) : squelette entier et en connexion anatomique d'un chien retrouvé dans un puits de la "nécropole punique» (Niveau de Villedary 2008, p. 124, lám. VII).

Des études archéozoologiques pourraient nous fournir des données fondamentales, surtout en ce qui concerne la mort des chiens et leur consommation éventuelle. Dans un ouvrage dédié à la nécropole punique de Cadix, Niveau de V. a examiné le dossier archéologique de ces puits ${ }^{95}$. Parfois, au lieu de chiens, il y avait d'autres animaux, entiers (surtout des cochons) ou non (surtout des Bos taurus, des Ovis/Capra et des thons), une grande quantité de mollusques (surtout des couteaux) et/ ou des squelettes humains entiers et en connexion anatomique qui, dans quelques cas, portaient des traces de mort violente. La localisation dans une nécropole, la conformation et le remplissage des puits indiquent effectivement une fonction rituelle de ceux-ci. Il reste toutefois difficile de déterminer plus précisément cette fonction, dans la mesure où l'on ignore le rapport entre les puits et les sépultures de la nécropole. Selon une première hypothèse, les puits pourraient être directement liés aux rites funéraires et contenir, donc, les restes de ces rites. Dans ce cas, les chiens révéleraient des sacrifices funéraires avec un but apotropaïque ou tutélaire, même si une consommation de ces animaux (quand ils sont représentés seulement par des portions de squelette) au cours des banquets sacrificiels ne peut pas être exclue. Il est également possible que ces puits n'aient pas été liés aux sépultures ni aux rites funéraires mais qu'ils aient été creusés dans la nécropole en raison des rites qui y étaient associés. Dans ce cas, les chiens (et les êtres humains) pourraient indiquer des sacrifices spécifiques (associés à une dimension chtonienne), ou, plus probablement, ils auraient été déposés dans les puits en tant que "morts atypiques" (à la suite, par exemple, d'une mort violente). Dans ce dernier cas, il n'y aurait pas de sacrifices mais des dépositions rituelles liées à des circonstances particulières.

Dans la même nécropole, des chiens entiers et en connexion anatomique ont été retrouvés au-dessous d'un mur (III ${ }^{\mathrm{e}}$ s.), au centre d'une structure circulaire en pierre (III ${ }^{\mathrm{e}} \mathrm{s}$.) et au-dessous d'une structure quadrangulaire en pierre ( $\mathrm{II}^{\mathrm{e}} \mathrm{s}$.). Dans ces cas, les chiens ont été interprétés comme des « sacrificios propiciatorios previos a la construcción/sacralización de las estructuras superpuestas; sin descartar su relación con cultos funerarios... ${ }^{96}$. Faute d'autres données sur ces trouvailles et d'éléments de comparaison dans le monde phénico-punique, il n'est pas possible à ce jour d'accepter ou de refuser cette proposition.

La découverte de chiens a été signalée pour d'autres nécropoles. Dans certains cas, comme à Alcácer do Sal (VII ${ }^{\mathrm{e}}-\mathrm{IV}^{\mathrm{e}}$ s.; la caractérisation phénicienne de cette nécropole reste débattue), les données disponibles sont insuffisantes pour pouvoir déterminer si ces restes avaient ou non une fonction rituelle $\left(\mathrm{n}^{\circ} 16\right)$. Dans d'autres cas, on sait au moins que les chiens avaient été retrouvés dans la sépulture ou dans la chambre funéraire: on peut citer, à ce propos, deux tombes à chambre localisées à Malte, respectivement à Mtarfa $\left(\mathrm{n}^{\circ} 2\right)$ et à Rabat $\left(\mathrm{n}^{\circ} 3\right)$, et une tombe à chambre de la nécropole de Villaricos $\left(\mathrm{V}^{\mathrm{e}}-\mathrm{II}^{\mathrm{e}} \mathrm{s}\right.$.; $\left.\mathrm{n}^{\mathrm{o}} 22\right)$ dans laquelle deux restes de Canis ont été retrouvés. Dans la nécropole de Cerro de la Tortuga (VI ${ }^{\mathrm{e}}-\mathrm{III}^{\mathrm{e}}$ s.; ${ }^{\circ} 19$ ), la présence d'un bon nombre de restes de chiens dans l'assemblage faunique (4\% des 1266 NRD) suggère que ces animaux avaient été utilisés lors de pratiques rituelles ${ }^{97}$.

Des trouvailles intéressantes ont été faites à Carthage. Les squelettes (apparemment complets)

96. Niveau de V. 2008, p. 128.

97. Uerpmann - Uerpmann 1973, p. 68-82. Au même titre que le sanctuaire, le caractère phénicien de cette nécropole reste débattu. 
d'un chien adulte et d'un jeune chiot ont été mis au jour dans une niche placée juste au-dessus de la chambre d'une tombe hypogéique à puits vertical localisée dans la nécropole de l'Odéon $\left(\mathrm{VI}^{\mathrm{e}}-\mathrm{V}^{\mathrm{e}} \mathrm{s} . ; \mathrm{n}^{\mathrm{o}} 5\right)$; des objets (deux galets polis noirs, un galet poli gris, etc.), des tessons de céramique, des coquillages, des débris d'oursins et des restes de Sus (une défense) et de Ovis/Capra (deux os longs) étaient associés aux chiens. Un seul individu humain, accompagné de plusieurs objets, avait été inhumé dans la chambre; dans les voisinages de la chambre, presque au niveau du sol vierge, il y avait des tombes d'enfants ensevelis dans des récipients en céramique. Le dépôt retrouvé dans la niche a sans doute un caractère rituel et les matériels qui en font partie, surtout les galets gris-noirs, évoquent une dimension chtonienne. Les chiens, qui n'avaient pas été consommés, pourraient représenter un sacrifice à valeur apotropaïque ou prophylactique lié au défunt enterré dans la chambre, même si on ne peut pas exclure qu'ils aient été liés plutôt aux enfants ${ }^{98}$. Une autre possibilité, à mon sens moins probable, serait que les deux chiens étaient les "gardiens " de la sépulture.

Il semble en revanche vraisemblable de proposer une fonction de gardiennage pour le chien d'une tombe fouillée en 1897 par A.L. Delattre dans la nécropole de Sainte-Monique $\left(\mathrm{V}^{\mathrm{e}}-\mathrm{II}^{\mathrm{e}} \mathrm{s.} ; \mathrm{n}^{\mathrm{0}} 7\right)$. Un puits vertical conduisait à deux chambres superposées. Dans la chambre du bas, devant l'entrée, il y avait le crâne d'un chien. Plusieurs tessons de céramique et quelques monnaies furent retrouvés à l'intérieur de la chambre, mais rien n'est dit sur le(s) défunt(s). La présence du crâne comme unique reste de l'animal suggère qu'il devait être porteur d'une valeur symbolique spécifique ${ }^{99}$.

Dans la même nécropole, quelques mois plus tard, Delattre fouilla une tombe tout à fait particulière $\left(\mathrm{n}^{\circ} 6\right)$ : dans une chambre localisée au fond d'un puits très profond (plus de $18 \mathrm{~m}$ ), une quarantaine de défunts avaient été inhumés avec plusieurs restes d'animaux et un riche mobilier d'accompagnement. Il s'agissait pour la plupart de jeunes gens et d'enfants des deux sexes, mais il y avait aussi deux personnages plus âgés conservant

98. Dans l'Antiquité l'association entre des enfants et des chiens est attestée dans plusieurs cas: voir la note 111 .

99. Cette trouvaille peut être comparée à celle de la nécropole de Sidon, qui a une chronologie semblable: voir la note 44 sur les ossements des traces rouges liées, selon Delattre, à la couleur des cercueils dans lesquels ils avaient originairement été déposés ${ }^{100}$. Les deux inscriptions funéraires retrouvées dans cette chambre, dédiées respectivement à un homme et à une femme, étaient probablement adressées à ces deux défunts ${ }^{101}$. À propos des restes d'animaux qui étaient mêlés aux restes humains, Delattre dit qu'il y avait des chiens, une énorme vertèbre qui paraît avoir appartenu à un cétacé et un autre os scié «d'un animal marin de grande taille» (dans les deux cas, il pourrait s'agir de baleines) $)^{102}$. Les photos publiées permettent de reconnaître sept crânes complets de chiens, un crâne de Sus et une mandibule de Ovis/Capra (fig. 65) ${ }^{103}$. On constate, comme à Sidon, la présence de sept crânes, qui doivent avoir une signification rituelle et symbolique bien précise et qui pourraient aussi avoir une valeur magique ${ }^{104}$. Selon Delattre, les animaux et les défunts plus jeunes avaient été tués rituellement pour accompagner les deux personnages plus âgés. Si cette proposition ne peut pas être écartée, il n'y a cependant aucun autre exemple semblable dans le monde phénico-punique.

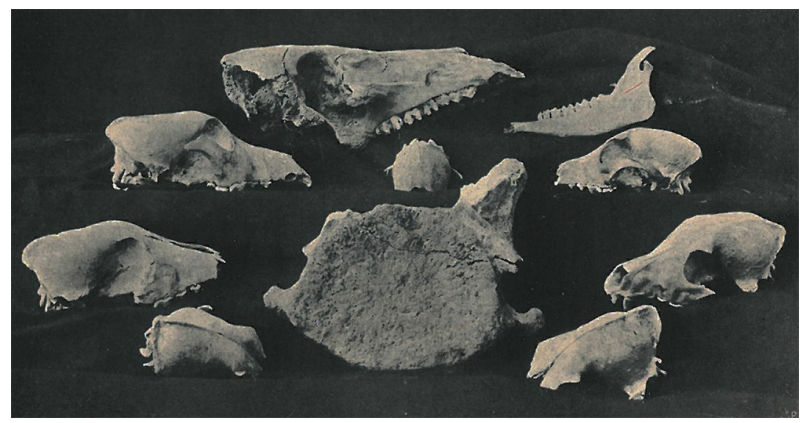

Fig. 6 - Carthage, nécropole de Sainte-Monique ( $V^{e}$ s.) : restes d'animaux (sept crânes de chiens, un crâne de Sus, une mandibule de Ovis/Capra et une vertèbre d'un cétacé) retrouvés dans la chambre funéraire d'une tombe hypogéique à puits vertical (Delattre 1905, p. 29, fig. 61).

100. Delattre 1905, p. 28. Il est également possible que la couleur ait dérivé de l'application de cinabre ou d'ocre rouge sur les cadavres, selon un usage attesté à Carthage et dans les nécropoles «libyco-phéniciennes» d'époque tardo-punique du Sahel (Bénichou-Safar 1982, p. 265-266).

101. Delattre 1905, p. 23, fig. 47. Ces inscriptions témoigneraient que les deux défunts n'étaient pas mariés.

102. Delattre 1905, p. 29, fig. 61-62.

103. Je remercie A. Nieto Espinet (CSIC - Conseil supérieur de la recherche scientifique) pour avoir identifié ces ossements.

104. Voir les considérations proposées quant aux trouvailles de Sidon. Cf. la note 114 (Genoni). 
Le chien dans la religion et dans la vie quotidienne des communautés phéniciennes et puniques

Dans une tombe à crémation primaire du $\mathrm{III}^{\mathrm{e}} \mathrm{s}$. de la nécropole de Solonte, une dent de chien a été retrouvée $\left(n^{\circ} 13\right)$ : elle pourrait soit avoir une fonction apotropaïque ou tutélaire, soit être intrusive. Cette dernière option peut être exclue pour une mandibule d'un canidé mise au jour dans un hypogée de Sulky dans lequel 22 individus ont été déposés de la fin du $\mathrm{IV}^{\mathrm{e}}$ au I ${ }^{\mathrm{er}} \mathrm{s}$. $\left(\mathrm{n}^{\mathrm{0}} 9\right)^{105}$. Cette mandibule, calcinée, était associée à une crémation secondaire et avait vraisemblablement été incinérée en même temps que le défunt. Le fait que seule cette portion de squelette ait été déposée dans la tombe peut indiquer un sacrifice partagé de type alimentaire, même si l'on ne peut exclure que l'animal entier ait fait partie du bûcher et n'ait donc pas fait l'objet d'une consommation humaine.

La découverte, comme à Cadix, de squelettes entiers et en connexion anatomique indique en soi qu'il ne s'agit pas de sacrifices partagés avec consommation de la viande. L'exemple le mieux connu est celui d'une tombe hypogéique fouillée à Malaga dans les années 2000-2001 dans laquelle quatre personnes (une femme, deux hommes et un individu indéterminé) avaient été inhumées entre le $\mathrm{VI}^{\mathrm{e}}$ s. et le début du IV ${ }^{\mathrm{e}}$ s. ( $\left.\mathrm{n}^{\mathrm{o}} 20\right)$. Devant la porte d'entrée de la chambre funéraire, dans une couche de cendres, le squelette entier (en connexion anatomique) et partiellement brûlé d'un chien a été retrouvé avec deux amphores fragmentaires, un pendentif en bronze et trois fragments de fer qui ne présentaient pas de traces de brûlure. L'animal, de taille moyenne et de plus d'un an, avait été tué, mais non consommé. Son dépôt paraît associé à la construction de la tombe: une fonction propitiatoire, apotropaïque ou de gardiennage peut donc lui être assignée.

Les squelettes de deux chiens ont été retrouvés, avec plusieurs tessons de céramique, juste au-dessus d'une inhumation en plein terre datant

105. Guirguis - Unali 2012, p. 2015-2016. Cette mandibule a été examinée par A. Gardeisen (UMR 5140, Montpellier) lors d'une mission qui s'est déroulée en mars 2017. Cette mission s'inscrit dans le projet "Animaux dans des contextes phénico-puniques de Méditerranée centrale" soutenu par le LabEx ARCHIMEDE. La mandibule appartient très probablement à un Canis familiaris (plutôt qu'à un renard), mais son état de conservation précaire ne permet pas d'avoir des certitudes. Je voudrais remercier M. Guirguis, directeur de la mission archéologique de Sulky et Monte Sirai, et S. Muscuso, directrice du musée archéologique de Sant'Antioco, pour leur disponibilité. du VII $-V^{\mathrm{e}}$ s., dans la nécropole (phénicienne?) de La Joya à Huelva ( $\left.{ }^{\circ} 15\right)$. Il s'agit, selon l'interprétation proposée par A.M. Niveau de Villedary, d'une offrande rituelle réalisée lors de la fermeture de la sépulture.

Plusieurs hypogées de la nécropole de Puig des Molins à Ibiza contenaient d'un à six squelettes de chiens entiers et en connexion anatomique $\left(n^{\circ}\right.$ 17). Les informations publiées à ce propos sont encore peu nombreuses. Toutefois, les os ne présenteraient pas d'altérations anthropiques liées à la consommation et au meurtre de ces animaux, alors que des traces de rognage et de morsure de carnivores ont été identifiées. En considérant que ces os avaient aussi des traces d'exposition aux agents atmosphériques, J.V. Morales Pérez estime que les chiens, après leur décès - éventuellement leur meurtre -, avaient été exposés pendant quelque temps dans un lieu différent avant d'être déposés dans les hypogées ${ }^{106}$. En 2016, au cours des fouilles de la nécropole punique de Villamar (IV ${ }^{\mathrm{e}}-\mathrm{II}^{\mathrm{e}}$ s.) dirigées par P. Bartoloni et E. Pompianu, les squelettes probablement entiers de deux chiens, un mâle et une femelle, ont été retrouvés dans une chambre funéraire contenant plusieurs cadavres $\left(n^{\circ} 8\right)^{107}$.

Le chien dans les contextes rituels: remarques conclusives

Les trouvailles examinées indiquent que les chiens étaient utilisés dans les pratiques rituelles phénico-puniques, essentiellement dans les contextes funéraires et dans une moindre mesure dans les sanctuaires. Ce constat, associé à la configuration des dépôts (quand il s'agit d'animaux entiers et en connexion anatomique), suggère que ces animaux étaient employés surtout dans des rites destinés à des divinités chtoniennes avec une valeur apotropaïque, prophylactique et tutélaire, même si on ne peut préciser ni la fonction exacte de ces rites ni si les chiens étaient impliqués aussi dans des rites magiques (comme les sept crânes de Carthage et de Sidon le suggèrent). Une interprétation analogue peut être avancée pour le dépôt dans la tombe de portions de squelette

106. Morales Pérez 2008, p. 24.

107. Je voudrais remercier E. Pompianu (Université de Sassari) de m’avoir communiqué ces données inédites. 
spécifiques comme le crâne et, probablement, la mandibule. Un rôle d'accompagnement du maître dans la tombe n'est jamais attesté avec certitude mais ne peut pas non plus être écarté. Certains des contextes examinés suggèrent que des chiens étaient parfois associés à des défunts "spéciaux" (se distinguant par leur statut ou par les circonstances de leur mort) pour lesquels des rites particuliers étaient nécessaires.

Le chien était parfois également employé, semble-t-il, dans des sacrifices alimentaires comportant un partage de la viande entre le(s) dieu(x) et $\mathrm{l}(\mathrm{es})$ homme(s). Au contraire, la fonction de nourriture pour le défunt n'est pas plus attestée que l'utilisation lors de sacrifices votifs, propitiatoires ou de fondation. Enfin, les données disponibles ne permettent pas d'associer des types spécifiques de chiens à des pratiques rituelles particulières, bien que cela paraisse vraisemblable.

Des remarques analogues sont valables pour la plupart des cultures méditerranéennes avec lesquelles les Phéniciens/Puniques ont interagi ${ }^{108}$. Au Levant et plus généralement au Proche-Orient, la présence du chien dans les contextes rituels de l'âge du Fer est très rare si l'on met à part les «sépultures/nécropoles» dont nous avons traité ${ }^{109}$. Dans le monde gréco-romain, des chiens sont parfois présents dans des contextes rituels (il s'agit souvent, comme dans le monde phénico-punique, de puits/bothroi) liés à des divinités chtoniennes et à des rites apotropaïques ou magiques, divinatoires et thérapeutiques; ils sont généralement associés à des moments de passage comme la naissance, la maladie et l'accouchement. Il y a également

108. Chez les Celtes et les Gaulois, au contraire de la plupart des autres "civilisations" de la Méditerranée, le chien semble avoir un rôle plutôt important dans les pratiques sacrificielles (Horard-Herbin 2014, p. 74-75) : il est présent dans des contextes religieux et est parfois utilisé dans des sacrifices alimentaires partagés. Dans plusieurs cas, des chiens étaient déposés en association avec des restes d'autres animaux ou des restes humains. Dans le monde égyptien, des canidés (chien et chacals) ont été mis au jour en association avec des sépultures humaines (en tant que compagnons du maître ou gardiens de la tombe) ou dans des nécropoles d'animaux (en tant que chiens sacrés): Bouvier-Closse 2001; Colin - Adam - Pranjic 2014 (cf. les notes 31-32). Chez les Hittites, des chiens (généralement des chiots) étaient impliqués dans des rites avec une fonction apotropaïque, de purification, magique et thérapeutique: Collins 1992.

109. Par ex. Wilkens 2000, p. 44 (Tell Afis). Pour un panorama général: Meir 2013, p. 12-15. quelques cas où ils sont employés dans des sacrifices partagés dédiés à des divinités célestes ${ }^{110}$. Dans les contextes funéraires, les chiens accompagnent généralement leur maître dans la sépulture et/ou montent la garde auprès de lui; de façon récurrente, ils sont associés à des enfants ${ }^{111}$.

Dans les contextes rituels des établissements non phéniciens de la péninsule Ibérique de l'âge du Fer, le chien est attesté plutôt rarement et dans quelques cas l'animal semble consommé lors de banquets sacrificiels ${ }^{112}$. Des dépôts rituels de chiens généralement entiers, associés ou non avec des restes d'autres animaux ou des restes humains, sont très rares à l'âge du Fer, alors qu'ils sont bien connus pour les époques plus anciennes, particulièrement au cours de l'âge du Bronze ${ }^{113}$. Le chien est encore plus rare dans les contextes religieux de la Sardaigne: à Genoni, dans un puits "sacré" nuragique réutilisé à l'époque impériale, on a retrouvé les squelettes entiers de sept chiens, dont l'un portait des traces de mort violente; des restes de Canis ont aussi été mis au jour dans le puits "sacré» nuragique de Serra Niedda, qui avait été utilisé entre la fin du II $^{\mathrm{e}}$ et le début du Ir millénaire ${ }^{114}$.

110. Pour les sources littéraires voir la note 30. Pour les sources archéologiques: Moore - Snyder 2006 (voir les contributions de I. Chenal-Velarde, S. Chilardi, J. De Grossi Mazzorin et C. Minniti, B. Wilkens); Lacam 2008; Raptou 2009, p. 100-101; G. Ekroth dans Campbell 2014, p. 339-34l. Concernant l'association entre les chiens et les sacrifices chtoniens avec but de purification, il est nécessaire d'éviter toute généralisation. En effet, cette prétendue association dérive parfois d'une conception apriorique de l'impureté du chien - conception qui s'est diffusée à la suite, surtout, des affirmations de Plutarque $(Q R, 68)$.

111. Voir, en plus des références de la note précédente, K. Trantalidou dans Moore - Snyder 2006, p. 96-119; Luce 2008; 2015. Plusieurs exemples de dépôts simultanés de chiens et d'enfants sont connus dans le monde gréco-romain (J. De Grossi Mazzorin et C. Minniti dans Moore Snyder 2006, p. 62-66; cf., pour Chypre: Raptou 2009), dans le monde égyptien (Colin - Adam - Pranjic 2014), mais aussi dans le Proche-Orient (Umm el-Marra, âge du Bronze) et dans la péninsule Ibérique (plusieurs cas à l'âge du Bronze et du Fer; voir les références bibliographiques des deux notes suivantes; pour un status quaestionis cf. Catagnano 2016).

112. Iborra Eres 2004, p. 363; Oliver Foix 2014, p. 48-51; Pons - Colominas - Saña 2016.

113. Liesau - Esparza Arroyo - Sánchez Polo 2014.

114. B. Wilkens dans Moore-Snyder 2006, p. 131-136. 
Le chien dans la religion et dans la vie quotidienne des communautés phéniciennes et puniques

204 Bruno D'AndreA

\section{LES SOURCES ICONOGRAPHIQUES}

L'étude des représentations iconographiques peut fournir des informations fondamentales sur les rôles "concrets" et symboliques du chien dans le monde phénico-punique. Toutefois, des questions méthodologiques sont à considérer.

- Il n'est pas toujours possible de distinguer les représentations concrètes du chien des représentations symboliques, dans la mesure où les deux dimensions sont souvent imbriquées.

- Ce dernier constat est tout particulièrement valable pour la sphère religieuse. Dans plusieurs cas, en effet, l'interprétation rituelle et religieuse des iconographies canines demeure incertaine.

- Il faudrait différencier les représentations iconographiques phéniciennes de celles qui ne sont pas phéniciennes, mais cela n'est pas toujours possible. Il faut aussi considérer l'origine - locale ou importée - des supports. La représentation $\mathrm{d}^{\prime}$ un thème iconographique non phénicien sur des objets de production locale/phénicienne implique la réception de ce thème mais non nécessairement de ses valeurs symboliques originaires, qui pourraient avoir été réélaborées localement. D'autre part, la présence d'une iconographie sur un objet d'importation n'indique pas nécessairement la compréhension de cette iconographie par l'acquéreur. En outre, la question des propriétaires des objets retrouvés dans les fouilles reste ouverte: un objet d'importation pourrait, par exemple, avoir appartenu en réalité à un «étranger».

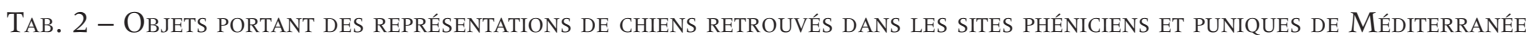
occidentale (Afrique du Nord, 1-35; Sardaigne, 36-53; Sicile, 54-58; péninsule Ibérique, 59-86); le coupes « PHÉNICIENNES » N $N^{\circ}$ 7 7-89 PROVIENNENT DE SITES ÉTRUSQUES DE L'ITALIE CENTRALE.

\begin{tabular}{|c|c|c|c|c|c|}
\hline $\mathrm{N}^{\circ}$ & Objet & Provenance & Chronologie & $\begin{array}{l}\text { Iconographie*/ } \\
\text { interprétation** }\end{array}$ & Bibliographie \\
\hline 1 & $\begin{array}{l}\text { Askos } \\
\text { (chien?) }\end{array}$ & $\begin{array}{l}\text { Carthage } \\
\text { (habitat) }\end{array}$ & VII' s. & $\begin{array}{l}\text { A } \\
5\end{array}$ & Giardino (à paraitre), pl. I, 7 \\
\hline $2-3$ & $\begin{array}{l}\text { Empreintes } \\
\text { de sceaux } \\
(2)\end{array}$ & $\begin{array}{l}\text { Carthage } \\
\text { (sanctuaire) }\end{array}$ & $\mathrm{VIII}^{\mathrm{e}}-\mathrm{V}^{\mathrm{e}} \mathrm{s}$ & $\begin{array}{l}\mathrm{D} \text { (procession d'ani- } \\
\text { maux) } \\
5 \text { (ou 3?) }\end{array}$ & Redissi 1999, p. 8-9, taf. IV, 40-41 \\
\hline 4 & $\begin{array}{l}\text { Scarabée en } \\
\text { jaspe vert }\end{array}$ & $\begin{array}{l}\text { Carthage } \\
\text { (nécropole) }\end{array}$ & VII $-\mathrm{VI}^{\mathrm{e}}$ s.? & $\begin{array}{l}\mathrm{D} \\
1 ?\end{array}$ & $\begin{array}{l}\text { L. Hautecour dans Drappier et al. } \\
\text { 1910, } \\
\text { p. } 357, \text { nº }^{\circ} 30, \text { pl. CIV }\end{array}$ \\
\hline 5 & $\begin{array}{l}\text { Scarabée en } \\
\text { jaspe vert }\end{array}$ & $\begin{array}{l}\text { Carthage } \\
\text { (nécropole) }\end{array}$ & VII $-\mathrm{III}^{\mathrm{e}} \mathrm{s}$. & $\begin{array}{l}\text { B ou C } \\
1 ?\end{array}$ & $\begin{array}{l}\text { Vercoutter } 1945, \text { p. } 227, \mathrm{n}^{\circ} 602 ; \\
\text { Boardman } 2003, \text { p. } 96,29 / 6\end{array}$ \\
\hline 6 & $\begin{array}{l}\text { Médaillon } \\
\text { en } \\
\text { terre cuite }\end{array}$ & $\begin{array}{l}\text { Carthage } \\
\text { (nécropole) }\end{array}$ & $\mathrm{VI}^{\mathrm{e}} \mathrm{s}$. & $\begin{array}{l}\text { B (ou C?) } \\
2(\text { ou } 1 ?)\end{array}$ & $\begin{array}{l}\text { Astruc 1959, p. 115-116, no } 18, \text { pl. } \\
\text { IV, 1; Yazidi 2009, p. 144, fig. } 3\end{array}$ \\
\hline 7 & $\begin{array}{l}\text { Moule en } \\
\text { terre cuite }\end{array}$ & $\begin{array}{l}\text { Utique } \\
\text { (nécropole) }\end{array}$ & $\mathrm{VI}^{\mathrm{e}} \mathrm{s}$ & $\begin{array}{l}\text { B (ou C?) } \\
2(\text { ou } 1 ?)\end{array}$ & Cintas 1951, p. 54-55, fig. 22 \\
\hline $\begin{array}{l}8-20 \\
(=\text { fig. } \\
6 \mathrm{E})\end{array}$ & $\begin{array}{l}\text { Empreintes } \\
\text { de sceaux } \\
(13)\end{array}$ & $\begin{array}{l}\text { Carthage } \\
\text { (sanctuaire) }\end{array}$ & $\mathrm{V}^{\mathrm{e}}-\mathrm{IV}^{\mathrm{e}}$ s.? & $\begin{array}{l}\text { A } \\
5 ?\end{array}$ & $\begin{array}{l}\text { Berges 1997, taf. XLII-XLIV, } \\
n^{\circ} 118-130\end{array}$ \\
\hline 21 & $\begin{array}{l}\text { Scarabée en } \\
\text { jaspe vert }\end{array}$ & $\begin{array}{l}\text { Carthage } \\
\text { (nécropole) }\end{array}$ & IV $^{\mathrm{e}} \mathrm{s}$ & $\begin{array}{l}\text { C et D } \\
5\end{array}$ & $\begin{array}{l}\text { Boardman 2003, p. 102, 32/24, pl. } \\
34 \text {; } \\
\text { Yazidi } 2009 \text {, p. 144-145, fig. } 4\end{array}$ \\
\hline 22 & $\begin{array}{l}\text { Scarabée en } \\
\text { jaspe vert }\end{array}$ & $\begin{array}{l}\text { Carthage } \\
\text { (nécropole) }\end{array}$ & IV ${ }^{\mathrm{e}} \mathrm{s}$ & $\begin{array}{l}\text { C (ou B?) } \\
5 ?\end{array}$ & $\begin{array}{l}\text { Vercoutter } 1945, \text { p. } 230, \mathrm{n}^{\circ} 616 \text {; } \\
\text { Yazidi } 2009, \text { p. } 146-147 \text {, fig. } 7\end{array}$ \\
\hline 23 & $\begin{array}{l}\text { Scarabée en } \\
\text { jaspe vert }\end{array}$ & $\begin{array}{l}\text { Kerkouane } \\
\text { (nécropole) }\end{array}$ & $\begin{array}{l}\text { Début } \\
\text { IV }^{\mathrm{e}} \mathrm{s} \text {. }\end{array}$ & $\begin{array}{l}\mathrm{B} \\
5\end{array}$ & $\begin{array}{l}\text { Redissi - Tillot 1995, p. 153, pl. } \\
\text { III, } 9 \\
\text { Yazidi } 2009, \text { p 148, fig. } 10\end{array}$ \\
\hline 24 & $\begin{array}{l}\text { Scarabée en } \\
\text { jaspe vert }\end{array}$ & $\begin{array}{l}\text { Kerkouane } \\
\text { (nécropole) }\end{array}$ & IVe $^{\mathrm{e}} \mathrm{s}$ & $\begin{array}{l}\text { B (ou C } ?) \\
1,3 \text { ou } 4 ?\end{array}$ & $\begin{array}{l}\text { Redissi - Tillot 1995, p. 136-137, } \\
\text { pl. XIII, 32; Yazidi 2009, p. 147, } \\
\text { fig. } 9\end{array}$ \\
\hline
\end{tabular}




\begin{tabular}{|c|c|c|c|c|c|}
\hline $\begin{array}{l}25 \\
(=\text { fig. } \\
6 \mathrm{H})\end{array}$ & $\begin{array}{l}\text { Rasoir en } \\
\text { bronze }\end{array}$ & $\begin{array}{l}\text { Carthage } \\
\text { (nécropole) }\end{array}$ & Fin $\mathrm{IV}^{\mathrm{e}} \mathrm{s}$ & $\begin{array}{l}\text { C (ou B?) } \\
5 ?\end{array}$ & $\begin{array}{l}\text { Picard 1966, p. 62-63, no } 13 \text {, pl. } \\
\text { XVIII, 53; Yazidi 2009, p. 146, fig. } \\
6\end{array}$ \\
\hline 26 & $\begin{array}{l}\text { Moule en } \\
\text { terre cuite }\end{array}$ & $\begin{array}{l}\text { Carthage } \\
\text { (sanctuaire) }\end{array}$ & $\mathrm{IV}^{\mathrm{e}}-\mathrm{II}^{\mathrm{e}} \mathrm{s}$ & $\begin{array}{l}\text { D (coq/poule, belette) } \\
3\end{array}$ & $\begin{array}{l}\text { Astruc 1959, p. 116-117, no20, pl. } \\
\text { IV, 3; Yazidi 2009, p. 148, fig. } 11\end{array}$ \\
\hline $\begin{array}{l}27 \\
(=\text { fig. } \\
6 \mathrm{D})\end{array}$ & $\begin{array}{l}\text { Bague en } \\
\text { bronze }\end{array}$ & $\begin{array}{l}\text { Carthage } \\
\text { (nécropole) }\end{array}$ & $\mathrm{IV}^{\mathrm{e}}-\mathrm{III} \mathrm{e}^{\mathrm{e}} \mathrm{s}$ & $\begin{array}{l}\mathrm{A} \\
5\end{array}$ & Yazidi 2009, p. 143, fig. 1 \\
\hline 28 & Bague en or & $\begin{array}{l}\text { Carthage } \\
\text { (nécropole) }\end{array}$ & $I^{e}-I I I I^{e} s$ & $\begin{array}{l}\text { A } \\
5\end{array}$ & Delattre 1906, p. 3 \\
\hline 29 & $\begin{array}{l}\text { Bague en } \\
\text { bronze }\end{array}$ & $\begin{array}{l}\text { Carthage } \\
\text { (nécropole) }\end{array}$ & $\mathrm{IV}^{\mathrm{e}}-\mathrm{II}^{\mathrm{e}} \mathrm{s}$ & $\begin{array}{l}\text { A } \\
5\end{array}$ & Delattre 1906, p. 26-27, fig. 54 \\
\hline $\begin{array}{l}30 \\
(=\text { fig. } \\
6 \mathrm{G})\end{array}$ & $\begin{array}{l}\text { Rasoir en } \\
\text { bronze }\end{array}$ & $\begin{array}{l}\text { Carthage } \\
\text { (nécropole) }\end{array}$ & $\mathrm{III}^{\mathrm{e}} \mathrm{s}$. & $\begin{array}{l}C \\
5\end{array}$ & $\begin{array}{l}\text { Picard 1964, p. 71-72, n 38, pl. } \\
\text { XXXI, 66; Yazidi 2009, p. 145, fig. } \\
5\end{array}$ \\
\hline 31 & $\begin{array}{l}\text { Amulette } \\
\text { en os }\end{array}$ & $\begin{array}{l}\text { Carthage } \\
\text { (nécropole) }\end{array}$ & $\mathrm{III}^{\mathrm{e}}-\mathrm{II}^{\mathrm{e}} \mathrm{s}$. & $\begin{array}{l}\text { A } \\
5\end{array}$ & $\begin{array}{l}\text { Berger 1900, p. 242-243, } \\
\text { n 27, pl. XXXIV }\end{array}$ \\
\hline 32 & Askos & $\begin{array}{l}\text { Bulla Regia } \\
\text { (nécropole) }\end{array}$ & $\mathrm{II}^{\mathrm{e}} \mathrm{s}$ & $\begin{array}{l}\mathrm{A} \\
5 \\
\end{array}$ & Giardino (à paraitre), pl. III, 3 \\
\hline 33 & Askos & \begin{tabular}{|l}
$\begin{array}{l}\text { Sousse } \\
\text { (nécropole) }\end{array}$ \\
\end{tabular} & $\mathrm{I}^{\mathrm{er}} \mathrm{s}$. & $\begin{array}{l}\text { A } \\
5\end{array}$ & Giardino (à paraitre), pl. III, 4 \\
\hline 34 & Askos & $\begin{array}{l}\text { Carthage } \\
\text { (nécropole) }\end{array}$ & $?$ & $\begin{array}{l}\text { A } \\
5\end{array}$ & $\begin{array}{l}\text { L. Poinssot dans Drappier et al. } \\
1910, \text { p. 157, n } 225\end{array}$ \\
\hline 35 & Amulette & \begin{tabular}{|l}
$\begin{array}{l}\text { Carthage } \\
\text { (nécropole) }\end{array}$ \\
\end{tabular} & $?$ & $\begin{array}{l}\mathrm{A} \\
5 \\
\end{array}$ & $\begin{array}{l}\text { L. Hautecœur dans Drappier et al. } \\
1910, \text { p. 350, nº } 159 \text {, pl. CV }\end{array}$ \\
\hline $36-41$ & $\begin{array}{l}\text { Amulettes } \\
\text { (6) } \\
\text { (chiens?) }\end{array}$ & Tharros & VII ${ }^{e}-$ III $^{e} s$. & $\begin{array}{l}\mathrm{A} \\
5\end{array}$ & $\begin{array}{l}\text { C. Mendleson dans Barnett - } \\
\text { Mendleson } \\
\text { 1987, p. 115, pl. 71, m }\end{array}$ \\
\hline 42 & $\begin{array}{l}\text { Scarabée en } \\
\text { cornaline }\end{array}$ & $\begin{array}{l}\text { Tharros } \\
\text { (nécropole) }\end{array}$ & $V^{e} s$. & $\begin{array}{l}\mathrm{C} \\
1\end{array}$ & $\begin{array}{l}\text { Boardman 2003, p. 80, 22/X52, } \\
\text { pl. } 56\end{array}$ \\
\hline 43 & $\begin{array}{l}\text { Scarabée en } \\
\text { jaspe vert }\end{array}$ & Tharros & $\mathrm{IV}^{\mathrm{e}}-\mathrm{III}{ }^{\mathrm{e}} \mathrm{s}$ & $\begin{array}{l}\text { B et } D \\
1\end{array}$ & $\begin{array}{l}\text { Boardman 2003, p. 82, 24/10, pl. } \\
23\end{array}$ \\
\hline 44 & $\begin{array}{l}\text { Scarabée en } \\
\text { jaspe vert }\end{array}$ & Tharros & $V^{e}-I V^{e} s$. & $\begin{array}{l}\text { D } \\
1 \text { (combat) } \\
\end{array}$ & Boardman 2003, p. 119, 39/37 \\
\hline 45 & $\begin{array}{l}\text { Scarabée en } \\
\text { jaspe vert } \\
\text { (chien?) }\end{array}$ & Tharros & $V^{e}-I I I^{e} s$. & $\begin{array}{l}\mathrm{B} \\
1 \text { ou } 3 ?\end{array}$ & $\begin{array}{l}\text { Boardman 2003, p. 96, 29/1 1, pl. } \\
30\end{array}$ \\
\hline 46 & $\begin{array}{l}\text { Statuette en } \\
\text { terre cuite }\end{array}$ & $\begin{array}{l}\text { Cagliari } \\
\text { (nécropole) }\end{array}$ & $\mathrm{IV}^{\mathrm{e}} \mathrm{s}$ & $\begin{array}{l}\text { A } \\
5\end{array}$ & Barreca 1986, p. 259, fig. 254 \\
\hline $\begin{array}{l}47 \\
(=\text { fig. } \\
6 \mathrm{C})\end{array}$ & Scarabée & Tharros & IV $^{\mathrm{e}}$ s.? & $\begin{array}{l}\text { B } \\
5\end{array}$ & $\begin{array}{l}\text { Redissi - Tillot 1995, p. 120, } \\
\text { pl. III, } 9\end{array}$ \\
\hline 48 & $\begin{array}{l}\text { Scarabée en } \\
\text { jaspe vert } \\
\text { (chien?) }\end{array}$ & Tharros & $\mathrm{IV}^{\mathrm{e}}-\mathrm{III} \mathrm{e}^{\mathrm{e}} \mathrm{s}$ & $\begin{array}{l}\mathrm{B} \\
1 ?\end{array}$ & $\begin{array}{l}\text { Boardman 2003, p. } 97,29 / 14, \text { pl. } \\
31\end{array}$ \\
\hline 49 & $\begin{array}{l}\text { Figurine en } \\
\text { terre cuite }\end{array}$ & \begin{tabular}{|l} 
Santa Gilla \\
(sanctuaire)
\end{tabular} & $I^{\mathrm{e}}-\mathrm{III}{ }^{\mathrm{e}} \mathrm{s}$ & $\begin{array}{l}\text { A } \\
5 ?\end{array}$ & Garbati 2008, p. 21, fig. 6 \\
\hline $50-52$ & $\begin{array}{l}\text { Scarabées } \\
\text { en } \\
\text { jaspe vert } \\
(3)\end{array}$ & Tharros & $?$ & $\begin{array}{l}\text { A } \\
5\end{array}$ & $\begin{array}{l}\text { Boardman 2003, p. 126, } \\
43 / 24-26 \text {, pl. } 47\end{array}$ \\
\hline 53 & $\begin{array}{l}\text { Scarabée en } \\
\text { jaspe vert }\end{array}$ & Tharros & $?$ & $\begin{array}{l}\text { B ou C? } \\
5 ?\end{array}$ & $\begin{array}{l}\text { Boardman 2003, p. 87, 26/40, pl. } \\
25\end{array}$ \\
\hline
\end{tabular}


Le chien dans la religion et dans la vie quotidienne des communautés phéniciennes et puniques

\begin{tabular}{|c|c|c|c|c|c|}
\hline 54 & Scarabée & $\begin{array}{l}\text { Palerme } \\
\text { (nécropole) }\end{array}$ & $\mathrm{VII}^{\mathrm{e}}-\mathrm{VI}^{\mathrm{e}} \mathrm{s}$. & $\begin{array}{l}\text { D (oiseau) } \\
?\end{array}$ & Tamburello 1982, p.46 \\
\hline $\begin{array}{l}55 \\
(=\text { fig. } \\
6 \mathrm{~F})\end{array}$ & $\begin{array}{l}\text { Monnaies } \\
\text { (plusieurs) }\end{array}$ & $\begin{array}{l}\text { Éryx, } \\
\text { Palerme, } \\
\text { Motyé, } \\
\text { Ségeste }\end{array}$ & $V^{e}-I V^{e} s$ & $\begin{array}{l}\text { A } \\
5\end{array}$ & $\begin{array}{l}\text { Hill 1903, p. 86-95; } \\
\text { Jenkins 1971 }\end{array}$ \\
\hline 56 & $\begin{array}{l}\text { Statuette en } \\
\text { terre cuite }\end{array}$ & $\begin{array}{l}\text { Motyé } \\
\text { (sanctuaire } \\
\text { "tophet») }\end{array}$ & IV $^{\mathrm{e}}-\mathrm{III}{ }^{\mathrm{e}} \mathrm{s}$ & $\begin{array}{l}\text { C (ou B?) } \\
5 \text { (ou } 1 ?)\end{array}$ & $\begin{array}{l}\text { F. Bevilacqua dans Mozia VII, } \\
\text { p. 115-116, tav. LXXXIX, } 2\end{array}$ \\
\hline 57 & $\begin{array}{l}\text { Statuette en } \\
\text { terre cuite } \\
\text { (chien?) }\end{array}$ & $\begin{array}{l}\text { Motyé } \\
\text { (sanctuaire) }\end{array}$ & $V^{e}-I V^{e} s$ & $\begin{array}{l}\text { A-C } \\
5 ?\end{array}$ & $\begin{array}{l}\text { L. Nigro dans Kothon } 2014 \text {, } \\
\text { p. 19, fig. } 27\end{array}$ \\
\hline 58 & $\begin{array}{l}\text { Empreinte } \\
\text { sur } \\
\text { une tuile }\end{array}$ & $\begin{array}{l}\text { Motyé } \\
\text { (habitat) }\end{array}$ & $?$ & $\begin{array}{l}\text { A } \\
5\end{array}$ & Whitaker 1921, p. 304-305, fig. 81 \\
\hline 59 & $\begin{array}{l}\text { Amulette } \\
\text { en os }\end{array}$ & $\begin{array}{l}\text { Villaricos } \\
\text { (nécropole) }\end{array}$ & VII $-{ }^{\mathrm{e}}-\mathrm{III}^{\mathrm{e}} \mathrm{s}$. & $\begin{array}{l}\text { A } \\
5\end{array}$ & Astruc 1951, p. 60, lám. XXXII, 30 \\
\hline $60-63$ & $\begin{array}{l}\text { Bagues en } \\
\text { bronze (4) }\end{array}$ & $\begin{array}{l}\text { La Algaida } \\
\text { (sanctuaire) }\end{array}$ & $V^{e}-I V^{e} s$ & $\begin{array}{l}\text { A } \\
5\end{array}$ & $\begin{array}{l}\text { Corzo Sánchez 2000, p. 159, } \\
\text { fig. } 9, \mathrm{n}^{\circ} 4,38,39,45\end{array}$ \\
\hline 64 & $\begin{array}{l}\text { Bague en } \\
\text { bronze } \\
\text { (chien?) }\end{array}$ & $\begin{array}{l}\text { La Algaida } \\
\text { (sanctuaire) }\end{array}$ & $V^{e}-I^{e} s$ & $\begin{array}{l}\text { A } \\
5\end{array}$ & $\begin{array}{l}\text { López Amador - Ruiz Gil } 2010 \text {, } \\
\text { p. } 278 \text {, fig. } 13\end{array}$ \\
\hline 65 & Petit bronze & $\begin{array}{l}\text { Alcácer do } \\
\text { Sal (sanc- } \\
\text { tuaire) }\end{array}$ & $V^{e}-I^{e} s$ & $\begin{array}{l}\text { A } \\
5\end{array}$ & Gomes 2008, p. 72 \\
\hline $66-75$ & $\begin{array}{l}\text { Amulettes } \\
\text { en os }(10)\end{array}$ & $\begin{array}{l}\text { Ibiza (né- } \\
\text { cropole/ } \\
\text { provenance } \\
\text { inconnue) }\end{array}$ & IV $^{\mathrm{e}}-\mathrm{III}^{\mathrm{e}} \mathrm{s}$ & $\begin{array}{l}\text { A } \\
5\end{array}$ & Fernández et al.2009, p. 142-146 \\
\hline 76 & $\begin{array}{l}\text { Scarabée en } \\
\text { jaspe vert } \\
\text { (chien?) }\end{array}$ & $\begin{array}{l}\text { Ibiza } \\
\text { (nécropole) }\end{array}$ & IV $^{\text {e }}$ s. ? & $\begin{array}{l}\text { C (ou B?) } \\
5 ?\end{array}$ & $\begin{array}{l}\text { Fernández et al.2015, p. 87-91, nº8, } \\
\text { fig. } 16\end{array}$ \\
\hline 77 & $\begin{array}{l}\text { Scarabée en } \\
\text { jaspe vert }\end{array}$ & Ibiza & $?$ & $\begin{array}{l}\text { A } \\
5\end{array}$ & $\begin{array}{l}\text { Boardman 2003, p. 126, 43/23, pl. } \\
47\end{array}$ \\
\hline $78-79$ & $\begin{array}{l}\text { Scarabées } \\
\text { en } \\
\text { jaspe vert } \\
(2) \\
\end{array}$ & Ibiza & $?$ & $\begin{array}{l}\text { C et D } \\
5\end{array}$ & $\begin{array}{l}\text { Boardman 2003, p. 102, } \\
32 / 18-19, \text { pl. } 34\end{array}$ \\
\hline 80 & $\begin{array}{l}\text { Scarabée en } \\
\text { jaspe vert }\end{array}$ & Ibiza & $?$ & $\begin{array}{l}\mathrm{B} \\
1 ?\end{array}$ & Boardman 2003, p. 96, 29/3, pl. 30 \\
\hline $\begin{array}{l}81 \\
(=\text { fig. } \\
6 \mathrm{~A})\end{array}$ & $\begin{array}{l}\text { Scarabée en } \\
\text { jaspe vert }\end{array}$ & Ibiza & $?$ & $\begin{array}{l}\text { B } \\
1\end{array}$ & Boardman 2003, p. 96, 29/4, pl. 30 \\
\hline 82 & $\begin{array}{l}\text { Scarabée en } \\
\text { jaspe vert }\end{array}$ & Ibiza & $?$ & $\begin{array}{l}\mathrm{B} \\
1\end{array}$ & Boardman 2003, p. 82, 24/6, pl. 23 \\
\hline $\begin{array}{l}83 \\
(=\text { fig. } \\
6 \mathrm{~B})\end{array}$ & $\begin{array}{l}\text { Scarabée en } \\
\text { jaspe vert }\end{array}$ & Ibiza & $?$ & $\begin{array}{l}\mathrm{B} \\
2\end{array}$ & $\begin{array}{l}\text { Boardman 2003, p. 94, 28/131, pl. } \\
30\end{array}$ \\
\hline 84 & $\begin{array}{l}\text { Scarabée en } \\
\text { calcédoine }\end{array}$ & $\begin{array}{l}\text { Ibiza } \\
\text { (nécropole) }\end{array}$ & $?$ & $\begin{array}{l}\mathrm{B} \\
4 ?\end{array}$ & $\begin{array}{l}\text { Fernández et al. 2015, p. 132-138, } \\
\mathrm{n}^{\circ} 16, \text { fig. } 24\end{array}$ \\
\hline 85 & Bague & Almuñécar & $?$ & $\begin{array}{l}\text { A } \\
5\end{array}$ & $\begin{array}{l}\text { Molina Fajardo 1986, p. } 211 \text {, fig. } \\
11,3\end{array}$ \\
\hline
\end{tabular}




\begin{tabular}{|l|l|l|l|l|l|}
\hline 86 & $\begin{array}{l}\text { Scarabée en } \\
\text { jaspe vert }\end{array}$ & $\begin{array}{l}\text { Gibraltar } \\
(\text { sanctuaire })\end{array}$ & $?$ & $\begin{array}{l}\text { B } \\
1\end{array}$ & Boardman 2003, p. 96, 29/1, pl. 30 \\
\hline 87 & $\begin{array}{l}\text { Coupe } \\
\text { en argent }\end{array}$ & $\begin{array}{l}\text { Préneste } \\
(\text { nécropole) }\end{array}$ & VII ${ }^{\mathrm{e}}$ s. & $\begin{array}{l}\text { B et D } \\
2 \text { (charognard) }\end{array}$ & Markoe 1985, p. 191, E2 \\
\hline 88 & $\begin{array}{l}\text { Coupe } \\
\text { en argent }\end{array}$ & $\begin{array}{l}\text { Cerveteri } \\
(\text { nécropole) }\end{array}$ & VII $^{\mathrm{e}}$ s. & $\begin{array}{l}\text { B et D } \\
1\end{array}$ & Markoe 1985, p. 194-196, E6 \\
\hline 89 & $\begin{array}{l}\text { Coupe en } \\
\text { or }\end{array}$ & Chiusi & $?$ & $\begin{array}{l}\text { B et D } \\
3\end{array}$ & Markoe 1985, p. 365, comp. 11 \\
\hline
\end{tabular}

* Iconographie: A, le chien est représenté seul; B, le chien est associé à un personnage humain; C, le chien est associé à une divinité; $\mathrm{D}$, le chien est associé à d'autres animaux.

** Interprétation: fonction "concrète", 1 (chasse); 2 (guerre); 3 (berger); 4 (compagnie); fonction "symbolique ", 5 (protection, gardiennage, association avec la divinité, etc.).

Le Tab. 2 rassemble des objets provenant d'établissements phénico-puniques de la Méditerranée occidentale sur lesquels sont représentés des chiens (fig. 7) ${ }^{115}$. Compte tenu des considérations précitées, on a délibérément laissé de côté les objets non phéniciens (comme les vases grecs) et les représentations de chiens liées à des personnages divins ou mythologiques non phéniciens (comme Anubis et Scylla). Ces dernières peuvent révéler l'adoption du culte de divinités "étrangères" ou leur association/identification à une divinité locale, mais ce n'est pas assuré. On peut citer l'exemple de deux statuettes en terre cuite retrouvées respectivement au tophet et au complexe religieux du Kothon de Motyé: toutes les deux sont apparemment de production gréco-sicéliote et sont caractérisées par l'association du chien à la déesse grecque Artémis $\left(\mathrm{n}^{\circ} 56-57\right)^{116}$. Leur découverte dans des sanctuaires puniques est significative mais reste à expliquer: elle pourrait indiquer qu'il y avait dans ces sanctuaires un culte d'Artémis ou, plus probablement, que cette déesse était associée et identifiée à une divinité phénicienne ${ }^{117}$. Il est aussi possible que ces

115. J'ai collecté, sans avoir la prétention de dresser un catalogue complet, tous les objets "phéniciens" avec une iconographie canine.

116. Je voudrais remercier N. Chiarenza (Université de Kiel) pour avoir attiré mon attention sur ces deux statuettes: voir Chiarenza (à paraître). Sur l'association fréquente entre Artémis et le chien dans le monde grec (attestée aussi par Plutarque, Moralia, 379 D) : K. Trantalidou dans Moore - Snyder 2006, p. 109-110.

117. Il pourrait s'agir de la divinité du tophet, Tinnit: une identification entre Tinnit et Artémis est attestée dans une théophore d'une inscription gréco-phénicienne découverte à Athènes (CIS $116=K A I 53)$. En tout cas, la représentation du chien est directement liée à celles d'Artémis et elle ne nous donne pas des renseignements sur l'image du chien chez les Phéniciens. statuettes aient été offertes par des individus de culture gréco-sicéliote.

D’une manière générale, dans le monde phénico-punique, les représentations iconographiques des chiens sont peu nombreuses par rapport à celles d'autres animaux, qu'ils soient domestiques (comme les boufs, les moutons et les chevaux) ou sauvages (comme les dauphins, les éléphants et les lions) ${ }^{118}$. Le chien n'est pas représenté (ou le serait très rarement) ${ }^{119}$ sur les stèles votives des tophets qui, par rapport à leur quantité (plus de 15000), sont la première source d'information. Cette absence peut être liée au manque presque absolu de restes de Canis dans les tophets et au fait que, chez les Phéniciens, le chien ne semble pas avoir été utilisé dans les sacrifices votifs (les inscriptions des tophets témoignent des vœux adressés à Baal Hammon et, éventuellement, à Tinnit).

On constate la même rareté des représentations de chiens chez les Phéniciens d'Orient et, plus généralement, au Levant et au Proche-Orient ${ }^{120}$. Il

118. Yazidi 2009.

119. Il y aurait des représentations de chiens sur quelques stèles carthaginoises (Hours-Miédan 1951, p. 49), mais en l'absence de reproductions graphiques de ces stèles il est impossible de vérifier cette information.

120. Collins 1992; T. Clark dans Brewer - Clark - Philips 2001, p. 49-80; Collins 2002 (voir, aux p. 79-236, les contributions de A.C. Gunter, P.F. Houlihan, C. Breniquet, M. Cool Root et A. Caubet); Miller 2008; Meir 2013. On peut citer quelques exemples "phéniciens»: Markoe 1985, p. 172-174, Cy4 (coupe en argent d'Amathonte, VIII ${ }^{\mathrm{e}}$-VII ${ }^{\mathrm{e}}$ s.: guerre); p. 357, comp. 3 (coupe en bronze de Nimrud, IX ${ }^{e}-\mathrm{VIII}^{\mathrm{e}} \mathrm{s}$.: chasse); A. Caubet dans Collins 2002, p. 219 (coupe en or de Ougarit, $\mathrm{XIV}^{\mathrm{e}}$-XIII ${ }^{\mathrm{e}}$ s.: chasse); Boardman 2003, p. 81, 23/Xl (scaraboïde en faïence de Kamid el-Loz, V ${ }^{e}-I^{e}$ s. : chasse); Sumaka'i Fink 2003, p. 48 (orthostate de Karatepe, IX ${ }^{\mathrm{e}}$-VIIII ${ }^{\mathrm{e}}$ s. : banquet, l'identification du chien demeure incertaine). Cf. les notes 45 (sarcophage de Sidon, IV ${ }^{\mathrm{e}}$ s.: chasse), 134, 139-141. 


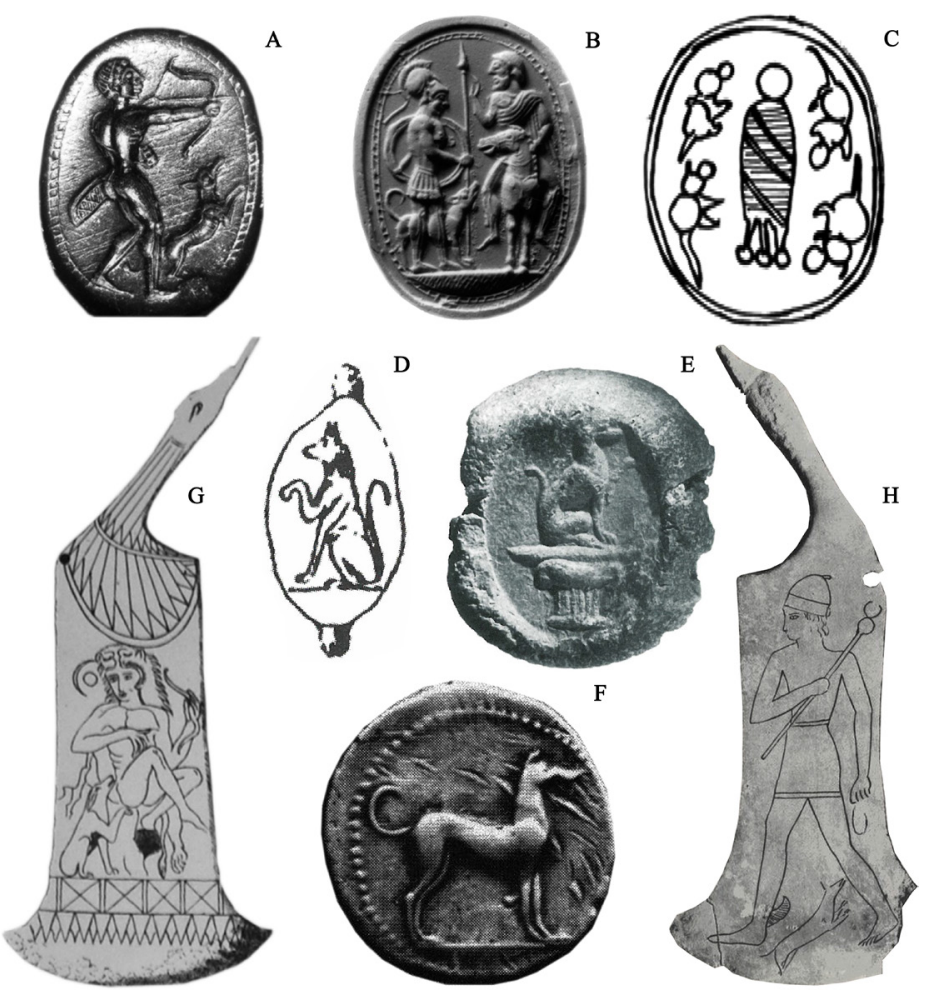

Fig. 7 - Objets portant des représentations de chiens retrouvés dans les sites phéniciens et puniques de Méditerranée occidentale: amulettes et scarabées, A-C; bague, D; empreinte de sceau, E; monnaie, F; rasoirs, G-H. Les images ne sont pas à l'échelle.

A ( =tab. III, n81) - Boardman 2003, 29/4, pl. 30. B (=n 83) - Boardman 2003, pl. 30, 28/131 (Classical Art Research Centre, http://www.beazley.ox.ac.uk/ gems/scarab/scarab.htm). C ( =n 47) - Redissi - Tillot 1995, p. 120, pl. III, 9. $D\left(=n^{\circ} 27\right)$ - Yazidi 2009, p. 143, fig. 1. E ( =n8) - Berges 1997, taf. XLII, 118. $F\left(=n^{\circ} 55\right)$ - Jenkins 1971, pl. III, 13. G (=n³0) - Vercoutter 1945, pl. XXVIII, 913. H ( =n²5) - L. Drappier dans Drappier et al. 1910, pl. LXII.

s'agit le plus souvent d'images liées au rôle concret de l'animal (chasse, guerre, commensal dans les banquets, etc.) et qui ne concernent pas directement la sphère religieuse. En Mésopotamie, le chien est associé à la déesse Gula et cette association figure sur plusieurs supports du $\mathrm{III}^{\mathrm{e}}$ au ${ }^{\mathrm{er}}$ millénaire ${ }^{121}$. En Égypte, le chien et surtout le chacal sont associés à Anubis, gardien des nécropoles et responsable des momifications ${ }^{122}$. Dans le monde gréco-romain, le chien est fréquemment représenté sur des supports différents ${ }^{123}$. Dans plusieurs cas, il est lié à la sphère funéraire, placé à côté de son maître, assurant un rôle de gardien ou d'accompagnateur dans l'au-delà; dans d'autres cas, il est associé à des personnages divins ou héroïques; très rarement, on le trouve impliqué dans des pratiques sacrificielles. Dans les établissements non phéniciens de la péninsule Ibérique à l'âge du Fer, le chien est représenté surtout dans des scènes de chasse et de dressage ${ }^{124}$.

Pour en revenir aux Phéniciens, il est intéressant de constater que la plupart des objets du tab. 2 proviennent de nécropoles. En effet, un bon nombre de ces objets est lié au monde funéraire avec une fonction apotropaïque, prophylactique et 
talismanique, comme dans le cas des amulettes et des scarabées qui sont de loin les objets sur lesquels le chien est le mieux attesté125. Une valeur tutélaire peut aussi être proposée pour une tuile retrouvée à Motyé ( $\mathrm{n}^{\circ} 58$; il pourrait s'agir plus simplement du timbre de l'atelier qui avait produit cette tuile), des askoi en forme de chien issus de contextes funéraires $\left(\mathrm{n}^{\circ} 32-34\right.$; le $\mathrm{n}^{\circ} 1$ provient d'un contexte domestique), une statuette retrouvée dans la nécropole de Tuvixeddu à Cagliari $\left(\mathrm{n}^{\circ} 46\right)$ et quelques bagues en bronze et en or $\left(\mathrm{n}^{\circ} 27-29,60-64,85\right)^{126}$. À propos des empreintes de sceaux découvertes à Carthage dans un complexe interprété comme un sanctuaire $\left(\mathrm{n}^{\circ} 2-3,8-20\right)$, on peut supposer que le sceau avait une fonction de garantie et de sauvegarde ${ }^{127}$.

Le chien est utilisé de façon symbolique dans le monnayage d'Éryx, Motyé et Palerme (n 55), qui toutefois empruntent cette iconographie à Ségeste, une cité élyme très proche ${ }^{128}$. L'iconographie du chien, parfois associé à un chasseur ou représenté en train de consommer la tête d'un cerf, s'affirme à Ségeste dans le dernier quart du $\mathrm{V}^{\mathrm{e}} \mathrm{s}$. et se diffuse rapidement dans les sites phénico-puniques de Sicile. Dans la même période, Carthage intervient militairement en Sicile pour aider les Ségestains en confirmant aussi, de cette manière, son hégémonie sur les autres établissements phéniciens de l'île ${ }^{129}$. L'utilisation du même motif iconographique par les quatre villes pourrait être directement liée aux événements historiques ${ }^{130}$. Le chien représenté sur les monnaies appartient à une race spécifique de la région ("cirneco dell'Etna») et il pourrait donc avoir une valeur d'auto-représentation et de "marqueur» régional par rapport, surtout, au territoire élyme ${ }^{131}$.

Sur plusieurs objets, la représentation du chien est clairement liée à ses fonctions dans la vie quotidienne (ce qui n'empêche pas que ces représentations puissent avoir aussi une signification symbolique): garde des troupeaux $\left(\mathrm{n}^{\circ} 26^{132}\right.$ et 89$)$, chasse $\left(\mathrm{n}^{\circ} 43,82^{133} ; 48,81,86\right.$ et, peutêtre, $5,44,80)^{134}$ et guerre $\left(\mathrm{n}^{\circ} 6-7^{135}, 83,88\right)^{136}$. Sur deux scarabées ( $\mathrm{n}^{\circ} 24$ et 45 , où l'identification de l'animal demeure incertaine), un chien est tenu en laisse par un personnage masculin: on ne peut pas déterminer s'il était associé à la chasse, à l'élevage ou s'il est représenté plus simplement en tant que compagnon de l'homme. La même question se pose pour un scarabée d'Ibiza, sur lequel un personnage masculin est en train de "jouer " avec un chien en le prenant par les pattes $\left(n^{\circ} 84\right)^{137}$.

L'animal est représenté sur quelques coupes "phéniciennes", mais il n'est pas certain que la production de ces objets soit à attribuer (seulement) aux artisans phéniciens ( $\mathrm{n}^{\circ}$ 87-89) ${ }^{138}$. Deux chiens qui se jettent de façon menaçante vers le corps d'un ennemi qui cherche à fuir sont représentés dans le registre central d'une coupe en argent retrouvée dans la tombe Bernardini à Préneste (680-670). Il s'agirait de la seule attestation phéni-
125. La plupart d'entre eux sont de production locale ou phénico-punique et leur iconographie est (ré)élaborée dans le monde phénicien. Il ne s'agit pas seulement d'imitation de thèmes figuratifs égyptiens, comme c'est souvent le cas pour ces objets. Cf. Vercoutter 1945; Boardman 2003.

126. On peut ajouter une bague de Tharros dans laquelle un chien avec une fleur de lotus dans la gueule est représenté (cela confirme le lien entre cet animal et la mort) : Pisano 1974, p. 41 (nº 114, fig. 3).

127. Des bulles semblables, remontant à l'époque de la domination carthaginoise (IV ${ }^{\mathrm{e}} \mathrm{s}$.), ont été retrouvées dans le temple C de Sélinonte: Salinas 1883, p. 310-311, n ${ }^{\circ}$ CCLXXVIICCLXXXVIII, tav. XIII. Cf. De Simone 2010, p. 26, fig. 13 (Héraclès en lutte contre un chien). Voir aussi De Simone 2008.

128. Hill 1903, p. 86-95; Jenkins 1971. Cf., pour Ségeste, Marconi 1997.

129. Melliti 2016, p. 77-82.

130. On pourrait supposer que le chien indiquait l'assujettissement et la fidélité à Carthage. Toutefois, il n'est pas possible d'accepter cette thèse en considérant que sur les monnaies de Ségeste le chien est originairement associé à un chasseur et que l'iconographie du chien est bien attestée dans le monnayage grec et gréco-sicéliote.
131. Marconi 1997, p. 1077-1078 et 1085-1086.

132. Quatre moules en terre cuite comparables à celui de Carthage ont été retrouvés à Ibiza: Mattazzi 2006, p. 149, $\mathrm{n}^{\circ}$ 16-19, tav. III.

133. Sur ces deux scarabées, le thème du personnage négroïde avec une gazelle sur les épaules est reproduit: Boardman 2003, p. 82.

134. Cf. un scarabée d'Ampurias (Boardman 2003, p. 97, 29/X1) et plusieurs scarabées de provenance inconnue (Boardman 2003, p. 96, 29/10, pl. 30; p. 69, pl. 19/14 et 18, pl. 18).

135. Sur ces deux objets, le chien est associé à un personnage armé montant un cheval (cf. un scarabée provenant d'Acre: Boardman 2003, p. 89, 27/21). Bien qu'on ait pu le proposer, il n'est pas possible de déterminer s'il s'agit d'un personnage mythique ou divin. Par ailleurs, il pourrait s'agir d'un chasseur et non d'un guerrier, même si la deuxième thèse est la plus vraisemblable.

136. Cf. un scarabée de provenance inconnue: Boardman 2003, p. $95,28 / \mathrm{X} 26$, pl. 58.

137. Mais il faut dire que, dans le monde phénico-punique, la fonction du chien comme "pet" n'est pas attestée.

138. Pour une étude de ces objets: Markoe 1985. Cf. la note 120. 
Le chien dans la religion et dans la vie quotidienne des communautés phéniciennes et puniques

cienne du chien "carnassier" et "charognard" - un thème qui est bien attesté dans les sources iconographiques et littéraires d'autres cultures méditerranéennes, par exemple dans la culture grecque $^{139}$. Dans le cas de la coupe de Préneste, ce thème pourrait avoir été emprunté à l'art égyptien, dont s'inspire l'iconographie générale de la coupe.

Dans quelques cas, le chien est associé à des divinités: Bès $\left(\mathrm{n}^{\circ} 42\right.$, le chien accompagne le dieu dans la chasse), Melqart ( $\mathrm{n}^{\circ} 30$ et, vraisemblablement, 21, 78, 79 ${ }^{140}$ ), peut-être Hermès (identifié par le caducée: $\left.\mathrm{n}^{\circ} 25\right)^{141}$ ainsi qu'une divinité marine (identifiée par le trident: $\mathrm{n}^{\circ} 22$ et 76 - sur ce dernier objet, l'identification du chien demeure incertaine; le scarabée $\mathrm{n}^{\circ} 53$ pourrait aussi faire référence à une divinité marine). Une fonction rituelle, probablement votive, peut être proposée pour une figurine en terre cuite découverte à Cagliari dans une aire sacrée d'époque tardo-punique ( $\mathrm{n}^{\circ} 49$ ) et pour un petit bronze du sanctuaire d'Alcácer do Sal $\left(n^{\circ} 65\right)$. Sur deux scarabées provenant de Kerkouane et de Tharros ( $\mathrm{n}^{\circ} 23$ et 47) figurent quatre chiens (dont l'identification reste douteuse) autour d'un personnage momifié ou enveloppé dans un linceul: ces chiens pourraient avoir une valeur apotropaïque, tutélaire ou psychopompe ${ }^{142}$ par rapport au défunt, dans lequel certains ont proposé de reconnaître Melqart comme dieu mourant et renaissant ${ }^{143}$.

Il est parfois malaisé de préciser la signification des représentations iconographiques canines: c'est surtout le cas quand l'animal est représenté seul $\left(\mathrm{n}^{\circ} 8-20^{144}, 27-29,31,35,50,52,58-59,64\right.$,

139. Franco 2003, p. 111-139; Luce 2008; Georgoudi 2015.

140. Sur ces amulettes, le chien est associé à Héraclès (Melqart?) : le dieu tient un lion entre ses mains (il s'agit vraisemblablement du lion de Némée) et s'apprête à le frapper avec un bâton. La même iconographie se retrouve sur des scarabées du Levant (Atlit et Sidon) ou de provenance inconnue: Boardman 2003, p. 102-104, 32/29, 31, 34, 49, X5, X6, X10, pl. 34-35. Cf. la note 127 .

141. On trouve une représentation semblable sur un scarabée d'Amrit: Boardman 2003, p. 105, 33/X4, pl. 59. L'association avec Hermès pourrait être liée au rôle psychopompe du dieu. Toutefois, le lien effectif entre le caducée et Hermès dans le monde phénico-punique reste incertain, de même que la divinité phénicienne à laquelle Hermès aurait été identifié: Lipiński 1995, p. 393-396.

142. Cette fonction du chien, bien attestée dans le monde gréco-romain, n'est toutefois pas assurée dans le monde phénico-punique: Niveau de Villedary 2008, p. 131.

143. Redissi - Tillot 1995, p. 121.

144. Dans deux ou trois de ces empreintes de sceaux, le chien
66-75, 77; dans le $\mathrm{n}^{0} 54$ il est associé à un oiseau). Des amulettes reproduisent la tête d'un animal qui pourrait être identifié à un chien $\left(\mathrm{n}^{0} 36-41\right)^{145}$. Sur deux bagues ( $\mathrm{n}^{\circ} 27$ et 85 ), une empreinte de sceau $\left(n^{\circ} 8\right)^{146}$, un scarabée $\left(n^{\circ} 22\right)$ et un rasoir $\left(n^{\circ} 30\right)$, un chien levant une ou les deux pattes antérieures est représenté. Ce geste, qui dans deux cas est dirigé vers des divinités ( $\mathrm{n}^{\circ} 22$ et 30 ), indique vraisemblablement l'assujettissement volontaire du chien à son maître et, dans les deux cas cités, aux dieux. Sur l'empreinte de sceau $\mathrm{n}^{\circ} 8$, le chien est assis au-dessus d'un autel ${ }^{147}$.

Dans l'ensemble, les sources iconographiques corroborent et complètent les autres sources documentaires examinées. Les fonctions prophylactiques, talismaniques et apotropaïques de cet animal, suggérées déjà par les données archéologiques, se trouvent confirmées. C'est de ces fonctions que découle la corrélation récurrente du chien avec le monde funéraire. Les sources iconographiques donnent aussi quelques informations sur l'association du chien à des divinités spécifiques, spécialement à Melqart. Dans quelques cas, cet animal est représenté sur des objets offerts vraisemblablement comme des ex-votos; cela pourrait dériver plutôt des valeurs symboliques assignées au chien que de son utilisation dans les sacrifices votifs, laquelle n'est pas attestée dans le monde phénico-punique.

\section{CONCLUSION}

Les données recueillies ne sont pas encore suffisamment nombreuses, mais permettent une première synthèse. Par ailleurs, il faut considérer que les sources mobilisées s'étalent sur près d'un millénaire et concernent une zone géographique étendue, du Levant à la Péninsule ibérique: les considérations proposées ci-dessous ont donc un caractère indicatif.

semble avoir une proie dans la bouche. Il s'agirait alors clairement d'une référence à la chasse.

145. Des amulettes semblables ont été retrouvées à Carthage et dans quelques sites de Sardaigne (C. Mendleson dans Barnett - Mendleson 1987, p. 115).

146. Berges 1997, taf. $42, \mathrm{n}^{\circ} 118$.

147. Cf. Salinas 1883, p. $311, \mathrm{n}^{\circ}$ CCLXXXVIII, tav. 13 (empreinte de sceau du temple C de Sélinonte, IV ${ }^{\mathrm{e}}$ s.). 
Dans le monde phénico-punique, comme dans la région levantine et plus généralement au Proche-Orient, le chien ne paraît pas avoir la même "importance sociale" qu'il acquiert dans le monde gréco-romain, même s'il entretient sans doute des rapports familiers avec l'homme dans ses activités quotidiennes. Cet animal joue un rôle secondaire dans les contextes rituels par rapport aux autres animaux domestiques chez les Phéniciens aussi bien que dans les autres « civilisations » méditerranéennes (mis à part probablement le monde celtique-gaulois). Cette moindre importance accordée au chien semble dictée par le fait que celui-ci n'est généralement pas employé dans les sacrifices de type alimentaire (avec consommation de la viande), même si cela arrive quelquefois chez les Phéniciens (comme ailleurs, par exemple chez les Grecs et les Romains). Ce constat est directement lié à la question de la cynophagie, qui est attestée chez les Phéniciens d'Occident mais ne peut être considérée comme un trait distinctif de la culture phénico-punique. La présence, relativement rare, du chien dans les banquets rituels constitue une confirmation de cette assertion.

Chez les Phéniciens, le chien était utilisé pour la garde des troupeaux, la chasse et la guerre. L'utilisation comme gardien des espaces publics (comme les sanctuaires) et privés (comme les maisons) n'est pas attestée avec certitude, mais elle est suggérée par la fonction symbolique tutélaire qui est accordée à l'animal. Les données disponibles n'indiquent pas d'usage du chien comme "pet». De fait, il n'y a pas de cas évidents où il accompagnerait son maître, et l'on ne trouve pas non plus de démonstration d'affection envers cet animal, pas plus que des représentations d'enfants en train de jouer avec leur chien ${ }^{148}$. C'est tout le contraire de ce qui se passe dans le monde gréco-romain et dans le monde égyptien, où les liens d'affection entre l'homme et le chien sont très bien attestés. Ces liens se manifestent dès le
III $-I^{\mathrm{e}}$ millénaire pour s'affirmer de plus en plus au cours de l'âge du Fer et, surtout, à l'époque hellénistique, quand se développe véritablement l'utilisation de certains chiens comme animaux de compagnie (pour le plaisir et l'amusement, et non pour un usage précis) ${ }^{149}$.

Le rôle du chien dans la vie quotidienne correspond directement à l'image que l'on se fait de lui, et plus précisément aux fonctions prophylactiques et talismaniques qui lui sont attribuées dans certaines représentations iconographiques et dans quelques contextes rituels (surtout de type funéraire). Les caractères du chien auxquels les Phéniciens semblent donner une grande valeur symbolique sont la fidélité et l'asservissement volontaire, qui constituent un modèle de comportement envers les hommes et aussi envers les divinités. L'utilisation du terme chien dans l'onomastique et son association avec les dieux sont vraisemblablement directement liées à ces caractères: dans la plupart des cas, le terme paraît donc avoir une connotation positive et non péjorative.

Le chien semble avoir un rapport privilégié avec Melqart, comme l'indiquent certaines représentations iconographiques et - peut-être - le mythe de la découverte de la pourpre. Cette association pourrait dériver du caractère tutélaire de Melqart et probablement de son expérience "chtonienne" de mort/disparition ${ }^{150}$. La dimension chtonienne du chien est implicitement confirmée par sa présence en contexte funéraire et par son association avec Anubis et Hermès, laquelle est attestée aussi dans le monde phénico-punique. Selon A.M. Niveau de Villedary, chez les Phéniciens d'Occident, l'animal était associé «a una divinidad femenina genérica, que comparte rasgos de otras muchas, tanto semitas como clásicas, con connotaciones funerarias, psicopompas y infernales, pero a la vez señora y garante de la naturaleza, virgen y madre ${ }^{151}$. Les données disponibles ne permettent pas de confirmer cette proposition qui me semble,

149. J. Brewer (p. 28-48) et A. Phillips (p. 93-94) dans Brewer - Clark - Philips 2001 ; Bouvier-Closse 2001 ; Franco 2003, p. 37-93; Colin - Adam - Pranjic 2014, p. 44-46; Luce 2015. L'introduction dans le monde grec des chiens maltais (animaux de compagnie par excellence) date du $\mathrm{VI}^{\mathrm{e}} \mathrm{s}$. Luce 2008, p. 273.

150. Lipi冈ski 1995, p. 226-243.

151. Niveau de Villedary 2008, p. 132. tion des iconographies grecques dans le monde phénicien d'Orient à l'époque hellénistique: Nitschke 2015; Oggiano 2015; Bonnet 2015, p. 367-411. 
Le chien dans la religion et dans la vie quotidienne des communautés phéniciennes et puniques 212 Bruno D'AndReA

en outre, une généralisation excessive ${ }^{152}$. La thèse de L.E. Stager sur le caractère sacré du chien chez les Phéniciens n'est pas non plus confirmée par la documentation directe.

L'utilisation du chien dans des sacrifices apotropaïques est suggérée par les trouvailles archéologiques. La fonction tutélaire et prophylactique s'accompagne probablement d'une fonction expiatoire et de purification. Cette dernière fonction n'est cependant pas attestée avec certitude et ne doit pas être généralisée pour tous les sacrifices de type non alimentaire. De même, on restera prudent sur la question des usages du chien dans d'éventuels rites magiques, divinatoires et thérapeutiques. De fait, ces usages, attestés dans d'autres cultures, sont suggérés seulement par des données extérieures au monde phénico-punique. Rien ne permet d'affirmer que le chien était parfois employé dans des sacrifices votifs ni qu'il était associé à des rites liés à l'accouchement et à la naissance, comme c'est le cas dans le monde romain.

Les utilisations et le symbolisme du chien chez les Phéniciens ne paraissent pas changer dans le temps, mais la question de la cynophagie, qui semblerait attestée seulement chez les Phéniciens d'Occident, reste ouverte. Les données contredisent la thèse d'une connotation négative et «impure» du chien dans le monde phénico-punique. Cette thèse s'appuie effectivement sur l'application aux Phéniciens de stéréotypes présents dans les cultures gréco-romaine et hébraïque (voire "sémitique») et non sur l'examen du dossier documentaire. Toutefois, on ne peut pas affirmer non plus que chez les Phéniciens le chien avait généralement une connotation positive: en effet, il semble très vraisemblable que des chiens différents (chiots vs chiens adultes, chiens domestiques vs chiens sauvages, etc.) aient eu des valeurs symboliques différentes selon leurs fonctions concrètes et leurs caractéristiques physiques et comportementales.

Pour conclure, il est nécessaire de réaffirmer la nécessité d'examiner dans son ensemble la documentation directe (archéologique, épigraphique, iconographique et éventuellement littéraire) pour développer nos thèses et nos interprétations. À l'inverse, il convient d'éviter d'élargir à l'excès la perspective comparatiste (s'appuyant, par exemple, sur les mondes mésopotamien ou gréco-romain) et d'utiliser une documentation indirecte ou extérieure. De fait, les données provenant de cultures différentes risquent de mener le chercheur à des généralisations abusives. Dans les études sur le chien dans l'Antiquité, les notions de pur et d'impur tout comme celles de positif et de négatif sont souvent des leitmotivs utilisés de manière trop générique, d'autant plus que dans la plupart des cultures concernées (la culture hébraïque postexilique mis à part), ils n'existaient pas comme des concepts absolus et immuables. En définitive, il n'est pas possible de reconstruire une image unitaire et générale du chien chez les Phéniciens dans la mesure où il n'existe pas «un » chien ni «un» monde phénico-punique, mais plusieurs chiens (qui toutefois sont bien distingués des autres animaux) et plusieurs communautés phénico-puniques (qui toutefois ont des éléments culturels et matériels en communs).
152. L'association des chiens à Astarté à Kition pourrait avoir seulement une valeur nominale, alors que l'association avec l'Astarté d'Éryx demeure conjecturale (les vases figurant des chiens cités dans Niveau de Villedary 2008, p. 113 ne proviennent pas d'Éryx). Du reste, cette association entre les chiens et Astarté pourrait dériver du lien entre le chien et Aphrodite dans le monde grec (J. De Grossi Mazzorin et C. Minniti dans Moore - Snyder 2006, p. 63-64; elle est attestée aussi sur une empreinte de sceau de Sélinonte: De Simone 2008, p. 35, fig. 11). À Motyé, l'association entre cet animal et Artémis est attestée, mais cela ne signifie pas nécessairement une identification de la déesse grecque à une divinité phénicienne (Tinnit) ni un lien de cette dernière avec le chien: voir les notes 116-117. 


\section{Bibliographie}

Amadasi Guzzo 1986 = M.G. Amadasi Guzzo, Scavi a Mozia: le iscrizioni, Rome, 1986 (Collezione di studi fenici, 22).

Arruda - Cardoso 2016 = A.M. Arruda, J.L. Cardoso, Faunas domésticas e rituais funerários em Alcácer do Sal, dans M. Serra, R. Villaça (dir.), Matar a fome, alimentar a alma, criar sociabilidades. Alimentação e comensalidade nas sociedades pré e proto-históricas, Coimbra, 2016, p. 193-217.

Astruc 1951 = M. Astruc, La necropolis de Villaricos, Madrid, 1951 (Informes y memorias, 25).

Astruc $1959=$ M. Astruc, Empreintes et reliefs carthaginois de terre cuite, dans MEFRA, 71-1, 1959. p. 107-134.

Aubet - Núñez - Trellisó 2014 = M.E. Aubet, F.J. Núñez, L. Trellisó (dir.), The Phoenician cemetery of Tyre-Al Bass, II, Beyrouth, 2014 (BAAL Hors-série, 9).

Barreca 1986 = F. Barreca, La civiltà fenicio-punica in Sardegna, Sassari, 1986 (Sardegna archeologica, 3).

Barnett - Mendleson 1987 = R.D. Barnett, C. Mendleson (dir.), Tharros: A catalogue of material in the British $m u$ seum from Phoenician and other tombs at Tharros, Sardinia, Londres, 1987.

Bénichou-Safar $1982=$ H. Bénichou-Safar, Les tombes puniques de Carthage: topographie, structures, inscriptions et rites funéraires, Paris, 1982.

Berger $1900=$ Ph. Berger, Musée Lavigerie de Saint-Louis de Carthage, Paris, 1900.

Berges $1997=$ D. Berges, Die Tonsiegel aus dem karthagischen Tempelarchiv, dans F. Rakob (dir.), Karthago II, Mainz, 1997, p. 10-244.

Boardman 2003 = J. Boardman, Classical Phoenician scarabs: a catalogue and study, Oxford, 2003 (BAR international series, 1190).

Bökönyi 1990 = S. Bökönyi, Kamid el-Loz. 12, Tierhaltung und Jagd: Tierknochenfunde der Ausgrabungen 1964 bis 1981, Bonn, 1990.

Bonnet $2015=$ C. Bonnet, Les Enfants de Cadmos. Le paysage religieux de la Phénicie hellénistique, Paris, 2015.

Bouvier-Closse $2001=\mathrm{S}$. Bouvier-Closse, Les sépultures des canidés dans l'Égypte ancienne, dans L. Bodson (dir.), La sépulture des animaux: concepts, usages et pratiques à travers le temps et l'espace, Liège, 2001, p. 55-75.

Brewer - Clark - Philips 2001 = D. Brewer, T. Clark, A. Phillips, Dogs in antiquity: Anubis to Cerberus. The origins of the domestic dog, Warminster, 2001.

Çakirlar et al. 2013 = C. Çakirlar et al., Persian period dog burials in the Levant: new evidence from Tell el-Burak and a reconsideration of the phenomenon, dans Archaeozoology of the Ancient Near East X. Proceedings of the $X^{\text {th }}$ international symposium on the archaeozoology of South-Western Asia and adjacent areas, Louvain-Paris-Walpole, 2013, p. 243-264.

Calascibetta $2009=$ A. M. G. Calascibetta, Nuovi dati sulla necropoli soluntina, dans C. Ampolo (dir.), Atti delle VI giornate internazionali di studi sull'area elima e la Sicilia occidentale nel contesto mediterraneo, Pise, 2009, p. 633643.
Camarós - Estévez 2010 = E. Camarós et J. Estévez, Los restos arqueozoológicos de mamíferos, dans J. Ramón Torres, J. Suárez Padilla, F. Villada Paredes (dir.), El asentamiento protohistórico de Ceuta, Ceuta, 2010, p. 377-399.

Campanella $2008=$ L. Campanella, Il cibo nel mondo fenicio e punico d'Occidente, Pise-Rome, 2008 (Collezione di studi fenici, 43).

Campbell $2014=$ G.L. Campbell (dir.), The Oxford handbook of animals in classical thought and life, Oxford, 2014.

Cardoso 2000 = J.L. Cardoso, Les mammiferes d'Abul, dans F. Mayet, C. Tavares da Silva (dir.), Le site phénicien d'Abul (Portugal): comptoir et sanctuaire, Paris, 2000, p. 281-291.

Cardoso - Gomes 1997 = J.L. Cardoso, M.V. Gomes, O consumo de cão, em contextos fenício-púnicos, no território português, dans Estudos Orientais, 6, 1997, p. 89-117.

Cardoso et al. $2016=$ J.L. Cardoso et al., What the people of Utica (Tunisia) ate at a banquet in the $9^{\text {th }}$ century BCE. Zooarchaeology of a North African early Phoenician settlement, dans Journal of archaeological science: reports, 8, 2016, p. 314-322.

Carenti - Wilkens 2006 = G. Carenti, B. Wilkens, La colonizzazione fenicia e punica e il suo influsso sulla fauna sarda, dans Sardinia, Corsica et Baleares Antiquae, 4, 2006, p. 173-186.

Castaños Ugarte $1994=$ P. M. Castaños Ugarte, Estudio de la fauna de la necrópolis de Villaricos, dans Archaeofauna, 3, 1994, p. 1-12.

Catagnano 2016 = V. Catagnano, Aproximación morfométrica y paleogenética al estudio de la variabilidad de Canis L. Familiaris en la Península Ibérica desde el neolítico hasta época romana y su contextualización en el ámbito del Mediterráneo occidental (tesis doctoral, Universitat Autònoma de Barcelona, 2016; http://www.tesisenred.net).

Cereijo Pecharromán - Patón Domínguez $1989=$ M. A. Cereijo Pecharromán, D. Patón Domínguez, Estudio sobre la fauna de vertebrados recuperada en el yacimiento tartésico de la calle del Puerto 6, dans Huelva Arqueológica, 10-11, 1989, p. 215-244.

Cereijo Pecharromán - Patón Domínguez $1990=$ M. A. Cereijo Pecharromán, D. Patón Domínguez, Excavation arqueológica en el solar $n^{\circ} 29$ de la calle Puerto de Huelva: informe sobre la fauna de vertebrados recuperada, dans Huelva arqueológica, 12, 1990, p. 79-106.

Chahoud 2015 = I. Chahoud, Reconstruire les pratiques alimentaires liées aux animaux dans les lieux de cultes Levantins au Bronze récent, dans Proceedings of the international symposium Beirut 2012 "Cult and ritual on the Levantine coast and its impact on the Eastern Mediterranean realm», Beyrouth, 2015 (BAAL Hors-série, 10), p. 5-32.

Chahoud - Vila 2011 = I. Chahoud, E. Vila, The role of animals in ancient Sidon: an overview of ongoing zooarchaeological studies, dans AHL, 34-35, 2011 -2012, p. 259-284.

Chéhab 1951-1952 = M. H. Chéhab, Les terres cuites de Kharayeb. Texte, Paris, 1951-1952 (Bulletin du Musée de Beyrouth, 10). 
Le chien dans la religion et dans la vie quotidienne des communautés phéniciennes et puniques 214 Bruno D'ANDREA

Chiarenza (à paraître) $=\mathrm{N}$. Chiarenza, La coroplastica votiva dalle campagne di scavi XXV-XXVIII dall'Area sacra del Kothon, dans L. Nigro (dir.), Mozia XIV, Rome, à paraître.

Ciasca et al. 1996 = A. Ciasca et al., Saggio sugli incinerati del tofet di Mozia, dans Vicino Oriente, 10, 1996, p. 317-346.

Cintas 1951 = P. Cintas, Deux campagnes de fouilles à Utique, dans Karthago, 2, 1951, p. 5-79.

CIS $=$ Corpus inscriptionum Semiticarum. Pars prima, Inscriptiones Phoenicias continens, Paris, 1881-1962.

Colin - Adam - Pranjic 2014 = F. Colin, F. Adam, I. Pranjic, Harpocrate au chien et les cadavres de Qasr'Allam. Perspectives sur le statut rituel des inhumations animales dans l'Égypte ancienne, dans Archimède, 1, 2014, p. 32-63.

Collins 1992 = J. B. Collins, The puppy in Hittite ritual, dans The Oriental Institute news and notes, 136, 1992, p. 1-6.

Collins 2002 = B. J. Collins (dir.), A History of the Animal World in the Ancient Near East, Boston-Leyde-Cologne, 2002 (Handbuch der Orientalistik, 64).

Corrado - Bonanno - Vella 2002 = A. Corrado, A. Bonanno, N. C. Vella, Bones and bowls: a preliminary interpretation of the faunal remains from the Punic levels in Area B, at the Temple of Tas-Silg, dans Sh. Jones O'Day, A. Ervynk, W. van Neer (dir.), Behaviour behind bones: The zooarchaeology of ritual, status and identity, Oxford, 2004, p. 47-53.

Correia 2015 = F. Correia, Enterramentos de cães na Quinta do Almaraz, dans G. Branco et al., Arqueologia de transiçao: o mundo funerário, Évora, 2015, p. 113-124.

Corzo Sánchez $2000=\mathrm{R}$. Corzo Sánchez, El santuario de La Algaida (Sanlúcar de Barrameda, Cádiz) y la formación de sus talleres artesanales, dans B. Costa, J.H. Fernández (dir.), Santuarios fenicio-púnicos en Iberia y su influencia en los cultos indígenas. XIV jornadas de arqueología fenicio-púnica, Ibiza, 2000, p. 147-183.

Crawley Quinn - Vella 2014 = J. Crawley Quinn, N.C. Vella (dir.), The Punic Mediterranean identities and identification from Phoenician settlement to Roman rule, Cambridge, 2014.

D'Andrea (à paraître) = B. D'Andrea, I sacrifici animali nelle pratiche cultuali dei tofet e dei santuari di Saturno: dalla tradizione fenicia all'età romana, dans Scienze dell'Antichità, 23.3 (Atti del seminario "Il sacrificio. Forme rituali, linguaggi e strutture sociali»), Rome, à paraître.

Davis 2006 = S. Davis, Faunal remains from Alcáçova de Santarém, Lisbonne, 2006 (Trabalhos de arqueologia, 43).

Davis $2007=$ S. Davis, Mammal and bird remains from the Iron age and Roman Periods at Castro Marim, Lisbonne, 2007 (Trabalhos de CIPA, 2007).

De Grossi Mazzorin - Battafarano 2012 = J. De Grossi Mazzorin, M. Battafarano, I resti faunistici provenienti dagli scavi di Tas Silg a Malta: testimonianze di pratiche rituali, dans J. De Grossi Mazzorin, D. Saccà, C. Tozzi (dir.), Atti del $6^{\circ}$ convegno nazionale di archeozoologia, Lucca, 2012, p. 357-363.

Delattre 1901 = A.L. Delattre, Carthage, nécropole punique voisine de Sainte-Monique: second mois des fouilles, février 1899 (extrait du Cosmos), Paris, 1901.
Delattre 1905 = A.L. Delattre, La nécropole des rabs, prêtres et prêtresses de Carthage: deuxième année des fouilles (extrait du Cosmos), Paris, 1905.

Delattre 1906 = A.L. Delattre, La nécropole des rabs, prêtres et prêtresses de Carthage: troisième année des fouilles (extrait du Cosmos), Paris, 1906.

Del Olmo Lete - Sanmartín 2003 = G. del Olmo Lete, J. Sanmartín (traduit par W.G.E. Watson), A dictionary of the Ugaritic language in the alphabetic tradition, Boston-Leyde, 2003 (Handbook of oriental studies, 67).

De Simone 2008 = R. De Simone, Tradizioni figurative greche nella "Selinunte punica»: le cretule del tempio $C$, dans M. Congiu et al. (dir.), Greci e Punici in Sicilia tra V e IV secolo a.C. Atti del IV Convegno di Studi (Caltanissetta 6- 7 ottobre 2007), Caltanissetta, 2008, p. 31-45.

De Simone $2010=\mathrm{R}$. De Simone, Le cretule del «tempio C»: motivi iconografici greci nella Selinunte punica, dans Bollettino di archeologia on-line, I (volume speciale), 2010 URL / http://www.bollettinodiarcheologiaonline.beniculturali.it/documenti/generale/3_DESIMONE.pdf

Detry - Cardoso - Bugalhão 2016 = C. Detry, J.L. Cardoso, J. Bugalhão, A alimentação em Lisboa no decurso da Idade do Ferro: resultados das escavações realizadas no núcleo arqueológico da Rua dos Correeiros, dans Spal, 25, 2016, p. 67-82.

Drappier et al. $1910=$ L. Drappier et al., Catalogue du musée Alaoui. Supplément, Paris, 1910 (Musées et collections archéologiques de l'Algérie et de la Tunisie, 15).

Ekroth - Wallenstein 2013 = G. Ekroth, J. Wallenstein (dir.), Bones, behaviour and belief: the zooarchaeological evidence as a source for ritual practice in Ancient Greece and beyond, Stockholm, 2013 (Skrifter utgivna av Svenska institutet $i$ Athen, 55).

Estaca Gómez et al. 2015 = V. Estaca Gómez et al., Zooarqueología de los macrovertebrados del yacimiento fenicio del Teatro Cómico (Cádiz), dans Spal, 24, 2015, p. 5576.

Farello 2000 = P. Farello, Reperti faunistici punici da Tharros, dans Atti del $2^{\circ}$ convegno nazionale di archeozoologia, Forlì, 2000, p. 293-300.

Fernández et al. 2009 = J. H. Fernández et al., Amuletos púnicos de hueso hallados en Ibiza, Ibiza, 2009 (Treballs del museu arqueològic d'Eivissa i Formentera, 62).

Fernández et al. 2015 = J. H. Fernández et al., Nuevos estudios sobre escarabeos hallados en Ibiza, Ibiza, 2015 (Treballs del Museu Arqueològic d'Eivissa i Formentera, 73).

Ferrer Albelda - Niveau de Villedary 2004 = E. Ferrer Albelda, A.M. Niveau de Villedary, Sacrificios de cánidos en la necrópolis púnica de Cádiz, dans Huelva Arqueológica, 20, 2004, p. 63-88.

Finkbeiner et al.1997 = U. Finkbeiner et al., Bey 020 . Preliminary report of the excavation 1995, dans BAAL, 2, 1997, p. 114-167.

Franco 2003 = C. Franco, Senza ritegno. Il cane e la donna nell'immaginario della Grecia antica, Bologne, 2003 (Antropologia del mondo antico, 1).

Gaillard 1942 = L. Gaillard, Réservoir à amphores et sépultures puniques de Carthage, dans BCTH, 1938-1940, p. 327-333. 
Garbati 2008 = G. Garbati, Religio votiva. Per un'interpretazione storico-religiosa delle terrecotte votive nella Sardegna punica e tardo-punica, Pise-Rome, 2008 (RStudFen, supplemento, 34).

Garbati - Pedrazzi 2015 = G. Garbati, T. Pedrazzi (dir.), Transformations and crisis in the Mediterranean. "Identity" and Interculturality in the Levant and Phoenician West during the $12^{\text {th }}-8^{\text {th }}$ centuries BCE, Rome, 2015 (RStudFen, supplemento, 42 ).

Garbati - Pedrazzi 2016 = G. Garbati, T. Pedrazzi (dir.), Transformations and crisis in the Mediterranean. "Identity" and interculturality in the Levant and Phoenician West during the $8^{\text {th }}-5^{\text {th }}$ centuries BCE, Rome, 2016 (RStudFen, supplemento, 44).

Gardeisen - Garcia Petit - Piquès 2015 = A. Gardeisen, L. Garcia Petit, G. Piquès, Synthèse sur le mobilier archéologique d'origine animale, dans A. Caubet, S. Fourrier (dir.), Kition-Bamboula VI, Lyon, 2015, p. 359-375 (Travaux de la Maison de l'Orient et de la Méditerranée, 67).

Georgoudi 2015 = S. Georgoudi, À propos de l'image du chien "carnassier" en Grèce et de la théorie de l'animal "impur», dans Donum natalicium digitaliter confectum Gregorio Nagy septuagenario a discipulis collegis familiaribus oblatum, 2015 URL: http://chs.harvard.edu/CHS/ article/display/4824\#noteref_n.29.

Giardino (à paraître) = S. Giardino, Vases zoomorphes phéniciens et puniques de l'Afrique du Nord: comparaisons, fonctionnalité et symbolisme, dans Actés du XIe colloque international "Histoire et archéologie de l'Afrique du Nord", Paris, à paraître.

Gomes 2008 = E.H.P. Gomes, Os ex-votos proto-históricos do Castelo de Alcácer do Sal (Tese de mestrado, Arqueologia, Universidade de Lisboa, 2008; http://repositorio. ul.pt/handle/10451/488).

Gomes - Tavares da Silva $1994=$ M.V. Gomes, C. Tavares da Silva, Garvão. Un sanctuaire protohistorique du sud du Portugal, dans Les dossiers d'archéologie, 198, 1994, p. 34-39.

Guirguis - Unali 2012 = M. Guirguis, A. Unali, Ipogei sulcitani tra età punica e romana: la Tomba Steri I, dans M.B. Cocco, A. Gavini, A. Ibba (dir.), L'Africa romana. Atti del XIX convegno di studio, Rome, 2012, p. 2011 2030.

Hamdi Bey - Reinach 1892 = O. Hamdi Bey, Th. Reinach, Une nécropole royale à Sidon, Paris, 1892.

Hill 1903 = G.F. Hill, Coins of ancient Sicily, Oxford, 1903.

Hoftijzer - Jongeling 1995 = J. Hoftijzer, K. Jongeling, Dictionary of the North-West Semitic inscriptions, LeydeNew York-Cologne, 1995 (Handbuch der Orientalistik, 21).

Horard-Herbin 2014 =M.-P. Horard-Herbin, La viande de chien à l'âge du Fer: quels individus pour quelles consommations?, dans Gallia, 71-72, 2014, p. 69-87.

Horwitz 2015 = L.K. Horwitz, Dog remains, dans R. Kletter, I. Ziffer, W. Zwickel (dir.), Yavneh II. The 'Temple Hill' repository pit, Göttingen, 2015 (Orbis Biblicus et Orientalis. Series archaeologica, 036), p. 145-149.

Hours-Miédan $1951=$ H. Hours-Miédan, Les représentations figurées sur les stèles de Carthage, dans Cahiers de Byrsa, 1, 1951, p. 15-160.
Iborra Eres 2001 = M.P. Iborra Eres, Gestión de recursos y economia. Estudio faunístico, dans C. Aranegui Gascó (dir.), Lixus. Colonia fenicia y ciudad púnico-mauritana, Rabat-Valence, 2001 (Saguntum, 4), p. 200-204.

Iborra Eres 2004 = M.P. Iborra Eres, La ganadería y la caza desde el Bronce Final hasta el Ibérico Final en el territorio valenciano, Valence, 2004.

Iborra Eres 2005 = M.P. Iborra Eres, El material faunístico, dans C. Aranegui Gascó, M. Habibi (dir.), Lixus 2. Ladera Sur, Valence, 2005, p. 229-239 (Saguntum, 6).

Iborra Eres 2010 = M.P. Iborra Eres, Los restos faunísticos, dans C. Aranegui Gascó, H. Hassini (dir.), Lixus 3. Area suroeste del sector monumental, Valence, 2010 (Saguntum, 8), p. 65-68.

Jenkins $1971=$ J.K. Jenkins, Coins of Punic Sicily. Part I, dans $S N R, 50,1971$, p. 25-78.

Jongeling 2008 = K. Jongeling, Handbook of Neo-Punic inscriptions, Tubingen, 2008.

$K A I=\mathrm{H}$. Donner, W. Rölling, Kanaanäische und Aramäische Inschriften, Wiesbaden, 1962-2002.

Kothon 2012 = L. Nigro, F. Spagnoli (dir.), Alle sorgenti del Kothon. Il rito a Mozia nell'Area sacra di Baal 'Addir - Poseidon, Rome, 2012 (Quaderni di archeologia fenicio-punica/CM, 2).

Kothon 2014 = L. Nigro (dir.), The so-called "Kothon» at Motya. The sacred pool of Baal 'Addir/Poseidon, Rome, 2014 (Quaderni di archeologia fenicio-punica/CM, 3).

Lacam 2008 = J.-Cl. Lacam, Le sacrifice du chien dans les communautés grecques, étrusques, italiques et romaines: approche comparatiste, dans MEFRA, 120-1, 2008, p. 29-80.

Liesau - Esparza Arroyo - Sánchez Polo 2014 = C. Liesau, Á. Esparza Arroyo, A. Sánchez Polo, ¿Huesos en la basura o depósito ritualizado? Los perros descuartizados de La Huelga, dans Zephirus, 74, 2014, p. 89-115.

Lipiński 1995 = Éd. Lipiński, Dieux et déesses de l'univers phénicien et punique, Louvain, 1995 (Studia Phoenicia, 14).

López Amador - Ruiz Gil 2010 = J.J. López Amador, J.A. Ruiz Gil, Las ofrendas del santuario púnico-gaditano de La Algaida, dans E. Mata Almonte (dir.), Cuaternario y Arqueología: homenaje a Francisco Giles Pacheco, Cádiz, 2010, p. 271-281.

Luce 2008 = J.-M. Luce, Quelques jalons pour une histoire du chien en Grèce antique, dans Pallas, 76, 2008, p. 261 293.

Luce $2015=$ J.-M. Luce, Les chiens dans l'antiquité grecque, dans C. Bellier, L. Cattelain, P. Cattelain (dir.), Chiens et chats dans la Préhistoire et l'Antiquité, Treignes, 2015, p. 61-68.

Marconi 1997 = C. Marconi, Storie di caccia in Sicilia occidentale, dans Seconde giornate internazionali di studi sull'area elima, Gibellina-Pise, 1997, p. 1071-1120.

Markoe $1985=$ G. Markoe, Phoenician bronzes and silver bowls from Cyprus and the Mediterranean, Berkley-Los Angeles-Londres, 1985 (University of California classical studies, 26).

Martín Ruiz - Pérez-Malumbres Landa - García Carretero 2005 = J.A. Martín Ruiz, A. Pérez-Malumbres Landa, J.R. García Carretero, Tumba de cámara de la necrópolis fenicia de Gibralfaro, dans RStudFen, 31-2, 2003, p. 139-160. 
Le chien dans la religion et dans la vie quotidienne des communautés phéniciennes et puniques 216 Bruno D'ANDREA

Mattazzi 2006 = P. Mattazzi, Repertorio iconografico delle matrici puniche in terracotta, dans G. Pisano (dir.), Varia iconographica ab Oriente ad Occidentem, Roma, 2006 (Studi punici, 14), p. 109-162.

Meir 2008 = E. Meir, The dog burials at Achaemenid Ashkelon revisited, dans Tel Aviv, 35, 2008, p. 267-282.

Meir 2013 = E. Meir, Dog cult in Persian period Judea, dans Ph. Ackerman-Lieberman, R. Zalashik (dir.), A Jew's best friend? The image of the dog throughout Jewish history, Padstow, 2013, p. 12-35.

Melliti 2016 = K. Melliti, Carthage. Histoire d'une métropole méditerranéenne, Paris, 2016.

Miller 2008 = G. D. Miller, Attitudes toward dogs in ancient Israel: A reassessment, dans Journal for the study of the Old Testament, 32-4, 2008, p. 487-500.

Molina Fajardo 1986 = F. Molina Fajardo, Almuñécar a la luz de los nuevos hallazgos fenicios, dans M. E. Aubet, G. del Olmo Lete (dir.), Los Fenicios en la Península Ibérica, I, Sabadell, 1986, p. 193-208.

Montero 1999 = M. Montero, Informe de arqueofauna, dans M.E. Aubet et al., Cerro del Villar I, Sevilla, 1999, p. 313-318.

Moore - Snyder 2006 = E.A. Moore, L.M. Snyder (dir.), Dogs and people in social, working, economic or symbolic interaction. Proceedings of the $9^{\text {th }}$ conference of the international council of archaeozoology, Oxford, 2006.

Morales et al. $1994=$ A. Morales et al., The mammals, dans A. Morales, C. Roselló (dir.), Castillo de Doña Blanca. Archaeo-environmental investigations in the bay of Cádiz, Oxford, 1994 (BAR international series, 593), p. 37-69.

Morales Muñiz et al. 1994 = A. Morales Muñiz et al., Analisis de la fauna de mamíferos del yacimiento tartéssico de la calle del Puerto $n^{\circ} 10$, dans J.P. Garrido, E.M. Orta (dir.), El hábitat antiguo de Huelva, periodos orientalizante y arcaico: la primera excavación arqueológica en la Calle del Puerto, Madrid, 1994, p. 265-320.

Morales Pérez 2003 = J.V. Morales Pérez, Estudio de la fauna de la cueva-santuario púnica de Es Culleram, dans Saguntum, 35, 2003, p. 113-122.

Morales Pérez 2008 = J.V. Morales Pérez, Zooarqueología en un contexto ritual: posibilidades de estudio y ejemplos de aplicación en el Mediterráneo, dans E. Ferrer Albelda, J. Mazuelos Pérez, J.L. Escacena Carrasco (dir.), De dioses y bestias: animales y religión en el Mundo Antiguo, Sevilla, 2008 (Spal, 11), p. 13-32.

Mozia VII = A. Bevilacqua et al., Mozia VII, Rome, 1972 (Studi semitici, 40).

Mozia XI = L. Nigro (dir.), Mozia XI. Il Tempio del Kothon, Rome, 2005 (Quaderni di archeologia fenicio-punica, 2).

Muñoz Gambero 2009 = J.M. Muñoz Gambero, El Cerro de la Tortuga: el templo y la necrópolis ibero-púnica de Málaga, Málaga, 2009.

Naster $1985=$ P. Naster, Le chien et le murex sur des monnaies de Tyr, dans NAC, 14, 1985, p. 257-260.

Nieto Espinet et al. 2017 = A. Nieto Espinet, Votive well or refuse tip? Chronicle of an abandonment: taphonomic stu$d y$ of the faunal remains of an iron age well-cistern, dans Historical Biology, 2017 (https:// doi.org/10.1080/0891 2963.2017.1370585).

Nitschke $2015=$ L. Nitschke, What is Phoenician about
Phoenician material culture in the Hellenistic period?, dans J. Aliquot, C. Bonnet (dir.), La Phénicie hellénistique. Actes du colloque international de Toulouse (18-20 février 2013), Lyon, 2015 (Topoi supplément, 13), p. 207-238.

Niveau de Villedary 2008 = A.M. Niveau de Villedary, ¿Compañero en la muerte o guía hacia el más allá? El perro en la liturgia funeraria púnica, dans E. Ferrer Albelda, J. Mazuelos Pérez, J. L. Escacena Carrasco (dir.), De dioses y bestias: animales y religión en el Mundo Antiguo, Sevilla, 2008 (Spal, 11), p. 97-141.

Niveau de Villedary 2009 = A.M. Niveau de Villedary, Ofrendas, banquetes y libaciones. El ritual funerario en la necrópolis púnica de Cádiz, Sevilla, 2009 (Spal, 12).

Nobis 1999 = G. Nobis, Die Tierreste von Karthago, dans F. Rakob (dir.), Karthago III, Mayence, 1999, p. 574632.

Nobis $2000=$ G. Nobis, Tierreste aus dem phönizischen Kition, dans von P. Åström, D. Sürenhagen (dir.), Periplus. Festschrift für Hans-Günter Buchholz zu seinem achtzigsten Geburtstag am 24. Dezember 1999, Jonsered, 2000 (Studies in Mediterranean archaeology, 127), p. 121-134.

Oggiano 2015 = I. Oggiano, Le sanctuaire de Kharayeb et l'évolution de l'imagerie phénicienne dans l'arrière-pays de Tyr, dans J. Aliquot, C. Bonnet (dir.), La Phénicie hellénistique. Actes du colloque international de Toulouse (18-20 février 2013), Lyon, 2015 (Topoi supplément, 13), p. 239-266.

Oliver Foix 2014 = A. Oliver Foix, Perros en el culto, la economía y el prestigio de los Iberos, dans Quaderns de prehistòria i arqueologia de Castelló, 32, 2014, p. 43-61.

Ornan $2004=\mathrm{T}$. Ornan, The goddess Gula and her dog, dans Israel museum studies in archaeology, 3, 2004, p. 13-30.

Pardo Barrionuevo 2015 = C.A. Pardo Barrionuevo, Economía y sociedad rural fenicia en el Mediterráneo occidental, Sevilla, 2015 (Historia y geografía, 280).

Peckham 1968 = B. Peckham, Notes on a fifth-century Phoenician inscription from Kition, Cyprus (CIS 86), dans Orientalia, 37-3, 1968, p. 304-324.

Picard 1966 = C. Picard, Sacra Punica. Étude sur les masques et rasoirs de Carthage, Paris, 1966 (Karthago, 13).

Pisano 1974 = G. Pisano, I gioielli fenici di Tharros nel museo nazionale di Cagliari, Rome, 1974 (Collezione di studi fenici, 3).

Pons - Colominas - Saña 2016 = E. Pons, L. Colominas, M. Saña, Dog sacrifice at the Protohistoric site of Mas Castellar, dans C.A. Murray (dir.), Diversity of sacrifice: Form and function of sacrificial practices in the ancient world and beyond, Albany, 2016, p. 191-209.

Poulain 1982 = Th. Poulain, Analyses ostéologiques et malacologiques, dans S. Lancel, J.-P. Morel, J.-P. Thuillier (dir.), Byrsa II, Paris, 1982 (Collection de l'École française de Rome, 41), p. 385-388.

Prevost $2006=\mathrm{V}$. Prevost, Les ragoûts de chien : prophylaxie, beauté et gastronomie, dans Manger au Maghreb. Partie I, Toulouse, 2006, p. 88-96.

Quinn 2017 = J. Crawley Quinn, In search of the Phoenicians, Princeton, 2017.

Raban-Gerstel et al. 2008 = N. Raban-Gerstel et al., Early Iron age Dor (Israel): A faunal perspective, dans BASO, 349,2008 , p. 25-59. 
Rakham 1996 = J. Rakham, Bey 006. Animal bones and site 1 microsample-preliminary report, dans BAAL, 1, 1996, p. 224-227.

Raptou 2009 = E. Raptou, Nouvelles pratiques funéraires à Paphos hellénistique et romaine, dans CCEC, 39, 2009 (Actes du colloque "Chypre à l'époque hellénistique et impériale. Recherches récentes et nouvelles découvertes", Paris, 25-26 septembre 2009), p. 89-112.

Redissi 1999 = T. Redissi, Étude des empreintes de sceaux de Carthage, dans F. Rakob (dir.), Karthago III, Mayence, 1999, p. 4-92.

Redissi - Tillot $1995=$ T. Redissi, M. Tillot, Étude des scarabées et scaraboïdes de Kerkouane, dans Reppal, 9, 1995, p. 115-173.

Ribichini 1998 = S. Ribichini, La proibizione del sacrificio umano cartaginese, dans M. Dietrich, I. Kottsieper (dir.), "Und Mose schrieb dieses Lied auf». Studien zum Alten Testament und zum Alten Orient, Münster, 1998, p. 655-668.

Ribichini $2004 a=$ S. Ribichini, Al servizio di Astarte. Ierodulia e prostituzione sacra nei culti fenici e punici, dans A. González Blanco, G. Matilla Séiquer, A. Egea Vivancos (dir.), El mundo púnico II, Murcia, 2004 (Estudios Orientales, 5-6), p. 55-68.

Ribichini $2004 \mathrm{~b}=\mathrm{S}$. Ribichini, Sui rituali funerari fenici e punici. Tra archeologia e storia delle religioni, dans A. Gonzalez Prats (dir.), El mundo funerario. Actas del III Seminario internacional sobre temas fenicios, Alicante, 2004, p. 43-76.

Riquelme Cantal 2001 = J.A. Riquelme Cantal, Ganadería fenicio-púnica: ensayo crítico de síntesis, dans B. Costa, J.H. Fernández Gómez (dir.), De la mar y de la tierra, producciones y productos fenicio-púnicos. XV jornadas de arqueología fenicio-púnica, Ibiza, 2001, p. 111-119.

Sader 2005 = H. Sader, Iron Age funerary stelae from Lebanon, Barcelone, 2005 (Cuadernos de arqueología mediterránea, 11).

Sagona 2002 = C. Sagona, The archaeology of Punic Malta, Louvain, 2002 (Ancient Near Eastern studies, 9).

Saidah 1967 = R. Saidah, Chronique, dans Bulletin du musée de Beyrouth, 20, 1967, p. 156-180.

Salinas 1883 = A. Salinas, Selinunte, dans NSA, 1883, p. 287-314.

Saña 1994 = M. Saña, Análisis zooarqueológico del Pozo $H X$ 1, dans J. Ramon (dir.), El pozo púnico del «Hort d'En Xim» (Eivissa), Ibiza, 1994, p. 71-81 (Trabajos del museo arqueológico de Ibiza, 32).

Schattner-Rieser $2009=$ U. Schattner-Rieser, Le chien dans la tradition juive des littératures bibliques et para- et postbiblique, dans Res antiquae, 6, 2009, p. 293-304.

Slopsma - van Wijngaarden-Bakker - Maliepaard $2009=$ J. Slopsma, L. van Wijngaarden-Bakker, R. Maliepaard, Animal remains from the Bir Massouda excavations 2000/2001 and other Carthaginian settlement contexts, dans Carthage studies, 3, 2009, p. 21-64.

Snyder - Klippel 2003 = L. M. Snyder, W.E. Klippel, From Lerna to Kastro: Further thoughts on dogs as food in ancient Greece, dans E. Kotjabopoulou et al., Zooarchaeology in
Greece: Recent advances, Londres, 2003 (British School at Athens studies, 9), p. 221-231.

Soergel $1968=$ E. Soergel, Die Tierknochen aus der altpunischen Faktorei von Toscanos, dans $\operatorname{MDAI}(M), 9,1968$, p. $111-115$.

Sorrentino 1993 = C. Sorrentino, Il materiale osteologico, dans C. Tronchetti (dir.), Lo scavo di via Brenta a Cagliari, Cagliari, 1993 (Quaderni, supplemento, 9). p. 158-169.

Sorrentino 2009 = C. Sorrentino, Il materiale osteologico animale, dans J. Bonetto, G. Falezza, A.R. Ghiotto (dir.), Nora. Il foro romano: i materiali romani e gli altri reperti, II.2, Padova, 2009, p. 891-903.

Stager $2008=$ L.E. Stager (dir.), Ashkelon I: introduction and overview (1985-2006), Winona Lake, 2008.

Sumaka'i Fink 2003 = A. Sumaka'i Fink, Why did yrh play the dog? Dogs in RS 24.258 (= KTU 1.114) and 4QMMT, dans Aula orientalis, 21, 2003, p. 35-61.

Tamburello 1982 = I. Tamburello, Magia e religiosità a Palermo punica, dans Sicilia archeologica, 49-50, 1982, p. 45-56.

Uerpmann - Uerpmann 1973 = H.P. Uerpmann, M. Uerpmann, Tierknochenfunde aus der phönizischen Faktorei von Toscanos und anderen phönizisch beeinflussten der Provinz Málaga in Südspanien, dans Studien über frühe Tierknochenfunde der Iberischen Halbinsel, 4, 1973, p. 35-100.

Vercoutter $1945=$ Les objets égyptiens et égyptisants du mobilier funéraire carthaginois, Paris, 1945 (Bibliothèque archéologique et historique, 40).

Vila 2008 = E. Vila, L'économie alimentaire carnée et le monde animal à Ras Shamra, dans Y. Calvet, M. Yon (dir.), Ougarit au Bronze Moyen et au Bronze Récent: actes du colloque international tenu à Lyon en novembre 2001, Lyon, 2008 (Travaux de la Maison de l'Orient et de la Méditerranée, 47), p. 169-179.

Weinstock 1995 = J. Weinstock, Some bone remains from Carthago, 1991 excavation season, dans H. Buitenhuis, H.P. Uerpmann (dir.) Archaeozoology of the Near East: Proceedings of the $2^{\text {nd }}$ international symposium on the archaeozoology of southwestern Asia and adjacent areas, Leyde, 1995, p. 113-118.

Whitaker 1921 = J. I. S. Whitaker, Motya: A Phoenician colony in Sicily, Londres, 1921.

Wijngaarden-Bakker 2007 = L.H. van WijngaardenBakker, The animal remains from Carthage, campaign 1993, dans H. Niemeyer, R. Docter, K. Schmidt (dir.), Karthago. Die Ergebnisse der Hamburger Grabung unter dem Decumanus Maximus, Mayence, 2007, p. 841-848.

Wilkens 2000 = B. Wilkens, Tell Afis (Siria) 1999. Rapporto preliminare sui resti faunistici, dans EVO, 22-23, 19992000, p. 43-46.

Wilkens $2006=$ B. Wilkens, Resti rituali dal santuario, dans E. Acquaro, B. Cerasetti (dir.), Pantelleria punica, Bologna, 2006, p. 259-275.

Xella 2014 = P. Xella, "Origini» e «identità»: riflessioni sul caso dei Fenici, dans MEFRA, 126-2, 2014, p. 381-391.

Yazidi 2009 = S.Z. Yazidi, Le bestiaire dans l'imaginaire des Puniques, Tunis, 2009. 
